\title{
Risk factors in lateral window sinus elevation surgery
}

\author{
Tiziano Testori $^{1,2,3}$ | Tommaso Weinstein ${ }^{4}$ | Silvio Taschieri ${ }^{5,6}$ | Stephen S. Wallace ${ }^{7,8}$ \\ ${ }^{1}$ Section of Implant Dentistry and Oral Rehabilitation, Department of Biomedical, Surgical and Dental Sciences, University of Milano, IRCCS, Galeazzi Institute, \\ Milan, Italy \\ ${ }^{2}$ Department of Periodontics and Oral Medicine, School of Dentistry, The University of Michigan, Ann Arbor, Michigan, USA \\ ${ }^{3}$ Private Practice, Como, Italy \\ ${ }^{4}$ Head of Diagnostic Department, Humanitas Dental Center, Humanitas Research Hospital, Milan, Italy \\ ${ }^{5}$ Department of Biomedical, Surgical and Dental Sciences, Faculty of Dentistry, University of Milan, Milan, Italy \\ ${ }^{6}$ IRCCS, Istituto Ortopedico Galeazzi, Milan, Italy \\ ${ }^{7}$ Department of Periodontics, Columbia University College of Dental Medicine, New York, New York, USA \\ ${ }^{8}$ Private Practice, Waterbury, Connecticut, USA
}

Correspondence

Stephen S. Wallace, Department of Periodontics, Columbia University College of Dental Medicine, New York, NY, USA.

Email: sswdds.sinus@sbcglobal.net

\section{1 | INTRODUCTION}

The maxillary sinus is the largest in the group of paranasal cavities that also include the ethmoidal, frontal, and sphenoidal sinuses. It is a cavity with a pyramidal shape, comprising a medial wall facing the nasal cavity, a posterior wall facing the maxillary tuberosity, a mesiovestibular wall containing the canine fossae, an upper wall (which is the orbit floor), and finally, a lower wall that is next to the alveolar process and forms the floor of the maxillary sinus itself (Figure 1). ${ }^{1}$

The maxillary sinus can be involved during implant rehabilitation if clinicians need to regenerate bone in maxillary posterior areas as a result of alveolar bone atrophy caused by the loss of posterior teeth and subsequent progressive maxillary sinus pneumatization. The reduction of vascularity and the absence of occlusal loads result in a buccopalatal reduction in bone volume. ${ }^{2-5}$ Maxillary sinus augmentation was first described by Tatum, in $1976,{ }^{6}$ and was subsequently published by Boyne \& James, in $1980 .^{7}$ The surgical procedure has been modified over the years and at the time of writing is considered a predictable treatment for the rehabilitation of atrophic maxillae. ${ }^{8}$ The selective use of bone-replacement grafts, textured implants, and barrier membranes has a positive effect on implant survival. Piezoelectric surgery, rather than rotary instruments, for lateral window preparation and membrane separation has been shown, in some studies, to reduce intraoperative complications. ${ }^{8}$ The reliability of the procedure depends on a detailed knowledge of the anatomy and an awareness of possible risk factors that may affect this surgical procedure. Therefore, the objectives of this paper are:
- to describe maxillary sinus anatomy and its surgical implications.

- to assess pathologic conditions that could be contraindications to maxillary sinus elevation.

- to describe behavioral conditions impairing maxillary sinus health.

- to diagnose and manage intraoperative and postoperative complications.

- to draw meaningful, clinical conclusions and provide recommendations to increase procedure predictability.

\section{2 | MAXILLARY SINUS ANATOMY AND ITS SURGICAL IMPLICATIONS}

The walls mostly involved in the sinus lift procedure using the lateral approach are the anterolateral walls and the medial walls. In some patients, the anterolateral wall may consist of a thin $(<1 \mathrm{~mm})$ cortical layer containing vessels, nerves, and antral septa or ridges. In others, the wall may be thicker, especially in brachy-type patients in whom cross-facial diameter has increased. In some instances, bone dehiscences may be observed and, if this is the case, the surgeon should be very careful to avoid perforation of the Schneiderian membrane during flap elevation. Moreover, if residual crestal bone is thin ( $<1.5 \mathrm{~mm}$, meaning that only cortical bone is present), effective graft regeneration may not be possible. ${ }^{9}$ This is confirmed by a retrospective evaluation of sinus lift procedures showing that less than $4 \mathrm{~mm}$ of residual crestal bone is a risk factor for implant survival. The 


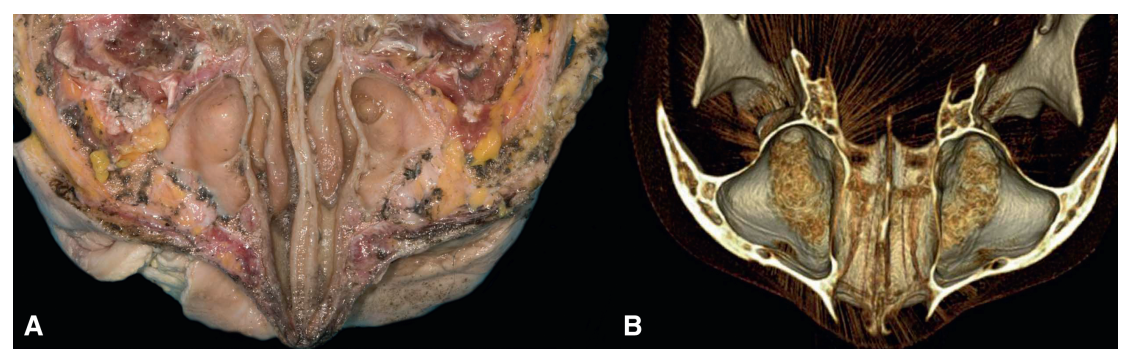

FIGURE 1 Transverse section of the middle third of the maxillofacial complex. It is characterized by a pneumatic cavity: the maxillary sinus. (A) Cadaver. (B) Conebeam computed tomography natural ostium is usually found in an anterosuperior location in the medial wall, and in $25 \%$ of patients an accessory ostium is present in the mucosal area called the anterior and posterior fontanelles. ${ }^{10}$ To prevent obliteration of the ostium (and creation of a subsequent complication), it is important not to elevate the sinus mucosa up to this point during sinus elevation procedures (Figures 2,3).

The maxillary sinus floor may have bone dehiscences around the roots of teeth adjacent to the area of the sinus elevation. In this situation, the apices could protrude into the sinus cavity covered only by the Schneiderian membrane and the surgeon should be aware of this anatomic variability. Furthermore, it should be taken into consideration that after tooth extraction it takes time for the indentations of the tooth roots in the maxillary sinus floor to remodel and for the floor to become naturally flat.

\subsection{The Schneiderian membrane}

The inner walls of the sinus are covered by the Schneiderian membrane, a pseudo-stratified, columnar, ciliated epithelium formed by basal, columnar, and calyx cells fixed to the basal membrane (Figure 4).

Pommer et al $^{11}$ recently described the mechanical properties of the Schneiderian membrane in humans in a cadaver study. The mean thickness of the membrane was determined to be $90 \pm 45 \mu \mathrm{m}$ (range: $24-350 \mu \mathrm{m}$ ). The mean burst elongation was $32.6 \pm 12.3 \%$ (range: $16.7 \%-74.7 \%$ ) in 1-dimensional testing and $24.7 \pm 4.7 \%$ (range: $15.2 \%-35.5 \%$ ) in 2-dimensional testing. This means that the membrane could be stretched to $132.6 \%$ of its original size in 1-dimensional elongation, and to $124.7 \%$ in 2-dimensional elongation. Thicker membranes demonstrated significantly higher stretching ability. A comparison between histologic findings and cone-beam computed tomography evaluation was made recently by Insua et al: ${ }^{12}$ for 597 membrane measurements, a mean Schneiderian membrane thickness of $0.30 \pm 0.17 \mathrm{~mm}$ was obtained histologically compared with $0.79 \pm 0.52 \mathrm{~mm}$ by cone-beam computed tomography. The difference was statistically significant as the value from the cone-beam computed tomography assessment was 2.6 times higher than that from the histologic examination.

Nevertheless, various pathologies could thicken the membrane as a result of inflammation. When thickening of more than $4 \mathrm{~mm}$ is found on a cone-beam computed tomography scan, an ear, nose, and throat consultation is recommended. A diseased, thickened membrane may have a gelatinous texture, especially in cases of hyperplastic-hypertrophic sinusitis, making the membrane weaker once the clinician has torn the periosteal layer during surgery. On the contrary, if the thickening is at the level of the periosteal layer, this will make the membrane stronger and less prone to perforation. The difference between a physiologically thickened membrane and a pathological one could be explained from a histological point of view: in the former the periosteum is thickened, whereas in the latter there is subepithelial inflammation in the middle layer (see Figure 4 for reference).

\section{2 | Maxillary sinus septa}

The presence of antral septa is recognized as a risk factor for Schneiderian membrane perforation during sinus elevation procedures. ${ }^{13}$ The septa are bony crests inside the sinus, first described by Underwood in 1910. ${ }^{14}$ They usually originate from the sinus floor and may stretch for a variable height on the lateral wall. Septa consist of a bone cortex, usually oriented in a vestibular-palatal direction, which
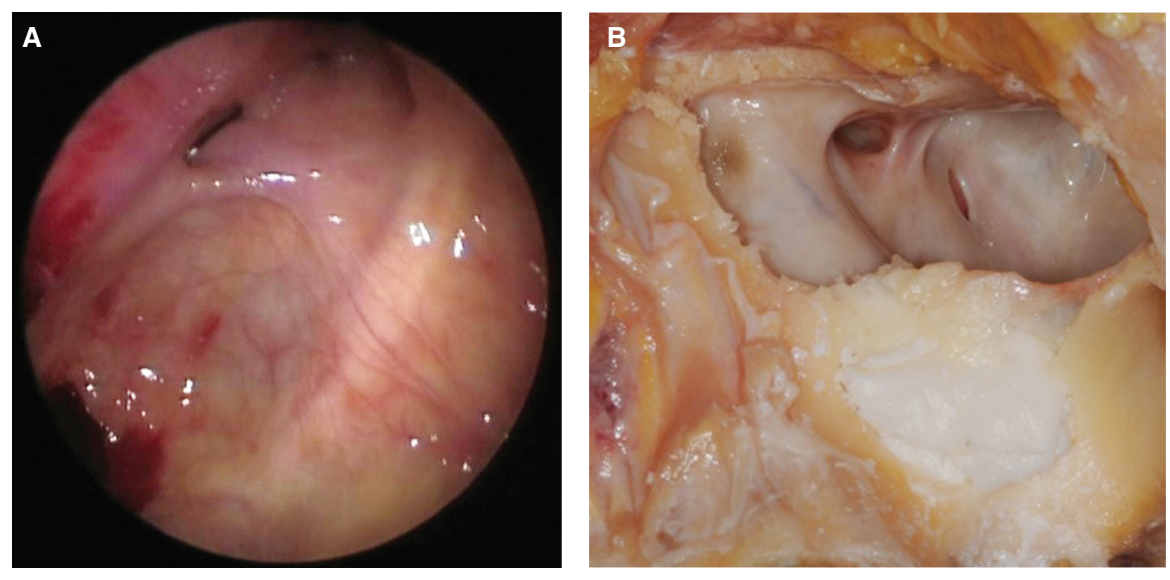

FIGURE 2 Ostium. (A) Endoscopic view. (B) Cadaveric view. The ostium is shaped like an oval or slit and is oriented horizontally or obliquely. The osteomeatal complex contributes to the final common drainage pathway of maxillary, anterior ethmoidal, and frontal sinuses 


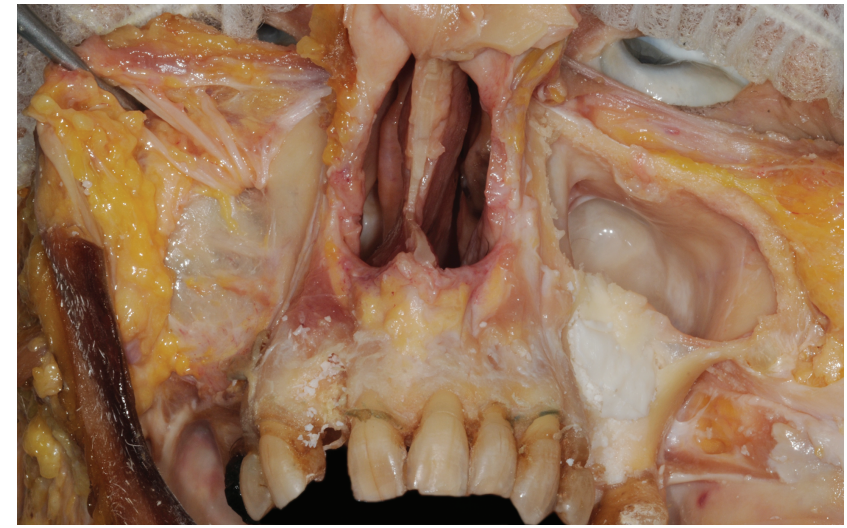

FIGURE 3 Cadaveric view. (left) Maxillary sinus. (right) Dissection of branches of infraorbital nerve

divides the distal part of the sinus into multiple compartments known as posterior recesses. Krenmair et $\mathrm{al}^{15}$ classified septa into primary (arising from development of the maxilla) and secondary (arising from irregular pneumatization of the maxillary sinus floor after tooth loss) and hypothesized that, because teeth are gradually lost, atrophy begins at different times in different regions.

The presence of septa and their dimension might influence the placement of dental implants during sinus augmentation procedures and could interfere with the shape of the anstrostomy. Various surgical approaches have been described to contend with this potential difficulty. The incidence of septa varies from $16 \%$ to $58 \%$ with an average of about $30 \% .{ }^{14,16-18}$ This large discrepancy in incidence is probably caused by varying inclusion criteria (ie, height) in reporting septal presence. Analysis of the literature showed that septa may be located in the anterior, middle, and posterior portions of the maxillary sinus, thus showing great variability. When septa are present, a surgical entry utilizing 2 small sinus antrostomies, placed anteriorly and posteriorly with respect to the septum location, could be considered. A second possibility would be to make a large window that extends over the septum to allow direct access to it from both the anterior and posterior aspects, for greater access and visibility. Symmetry of septa between contralateral sinuses was observed ${ }^{17}$, but complete separation of the sinuses was not. It is possible, however, that 2 separate compartments may exist at the working level of a sinus augmentation procedure. In these situations, variations in surgical approach are essential to avoid laceration of the Schneiderian membrane during its elevation. Presurgical knowledge of the anatomy, extension, and origin of existing septa is essential for proper navigation during membrane elevation. Currently, computed tomography is the method preferred for preoperative detection of septa and other anatomic variations in patients undergoing sinus surgery. ${ }^{17}$

\section{3 | Maxillary sinus arterial supply}

The vascular network of the maxillary sinus should be fully understood in order to avoid potential complications during sinus lift surgery (Figure 5A,B).
A
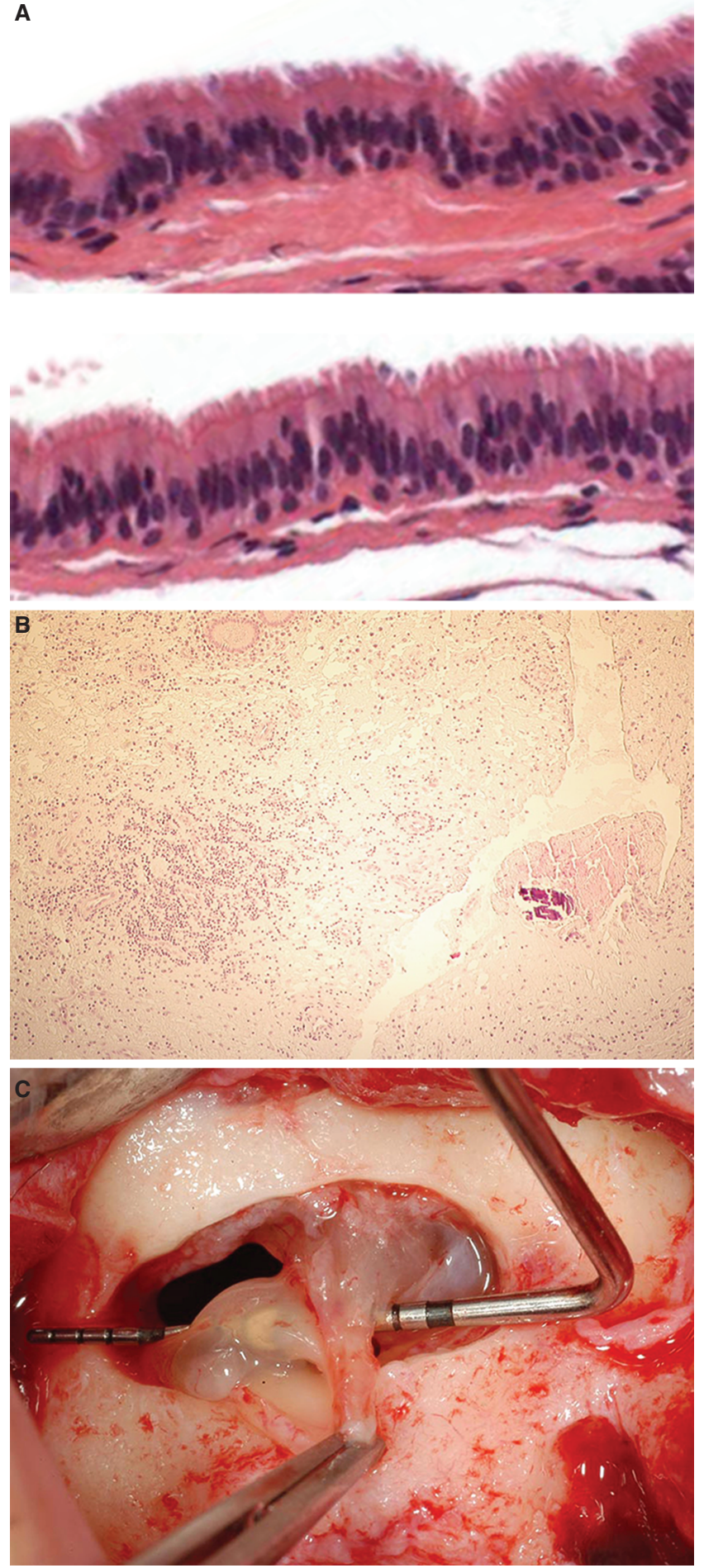

FIGURE 4 Schneiderian membrane. (A) Healthy membrane. Histology Courtesy of Rodella LF, University of Brescia, Italy. (B) Subepithelial inflammation Histology Courtesy of Valduce Hospital, Como, Italy. (C) Hypertrophic membrane with gelatinous consistency as a result of inflammation

Blood is supplied to the maxillary sinus through 3 main arteries (the infraorbital artery, the posterior lateral nasal artery, and the posterior superior alveolar artery), which are ramifications of the maxillary artery. Vascularization of the anterolateral wall of the sinus 


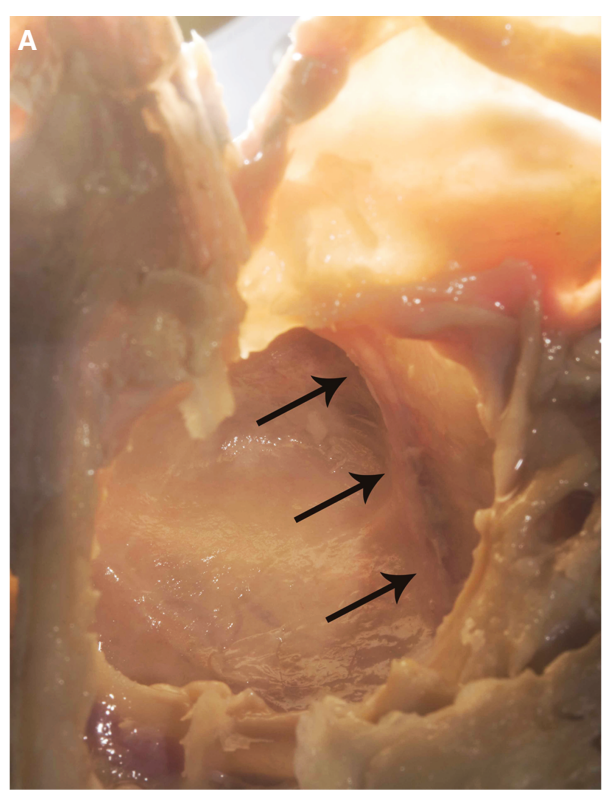

FIGURE 5 (A) Internal view of intraosseous anastomosis between the dental branch of the posterior superior alveolar artery and the infraorbital artery. The artery (arrows) is, between the Schneiderian membrane and the bony wall in the sinus antrostomy area, subperiosteal in the maxillary tuberosity area. (B) Anatomic dissection in the antrostomy region

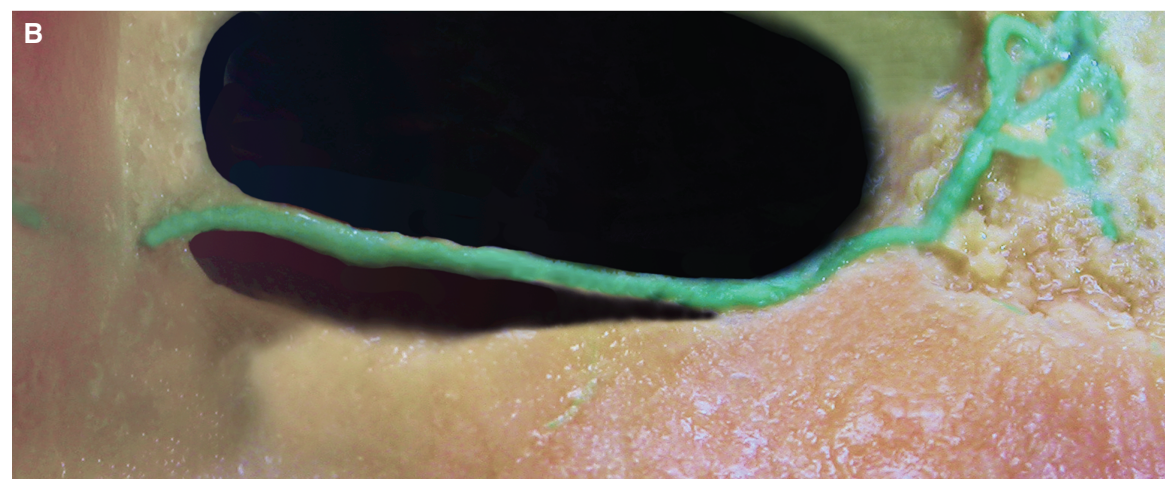

is characterized by the presence of the alveolar antral artery, an intraosseous anastomosis between the dental branch of the posterior superior alveolar artery and the infraorbital artery. ${ }^{19,20}$ This intraosseous anastomosis courses halfway up the lateral sinus wall and is present in the cortical bone of the lateral wall of the maxillary sinus in $100 \%$ of patients. ${ }^{20-22}$ However, from a radiographic point of view, it is evident only in approximately $50 \%$ of patients. ${ }^{23,24}$

The alveolar antral artery, the diameter of which has been reported by Elian et al, ${ }^{23}$ Mardinger et $\mathrm{al}^{24}$ and Testori et $\mathrm{al}^{25}$ to be up to $2.5-3 \mathrm{~mm}$, has the potential to cause bleeding complications during lateral window osteotomies. The transection of such an artery is not life threatening because its hemorrhage mostly self-resolves owing to a reactive contraction of the vessel. ${ }^{20}$ Nevertheless, impairment in visualization of the surgical field, especially when the diameter is wide, ${ }^{25}$ could interfere with both membrane elevation and placement of the graft material. If this complication occurs, it is important to avoid electrocautery as this may perforate the Schneiderian membrane and compromise the healing and remodeling of the sinus graft. Hemostasis can be obtained spontaneously or by pressure with a moistened gauze pad. More aggressive bleeding may be treated with bone wax or application of hemostatic compression.

An intraosseous anastomosis between the alveolar antral artery and the infraorbital artery was found by dissection in $100 \%$
(30/30 sinuses) of the anatomic cases, while a well-defined bony canal, located in the context of the sinus anterolateral wall, was detected radiographically in 94 (47\%) of 200 sinuses examined. ${ }^{20}$ The diameter of the bony canals was $<1 \mathrm{~mm}$ in 52 sinuses $(55.3 \%$ of 94 cases), $1-2 \mathrm{~mm}$ in 38 (40.4\%) sinuses, and $\geq 2 \mathrm{~mm}$ in 4 (4.3\%) sinuses. ${ }^{20}$ The alveolar antral artery displayed 3 different courses: (a) within the buccal antral wall cortex; (b) between the Schneiderian membrane and the lateral bony wall of the sinus, in which a small concavity was often visible; and (c) under the periosteum outside the lateral wall of the sinus. The above mentioned three different courses find alveolar antral artery respectively: (a) completely intraosseous at its extremities in 100\% of cases; (b) partially intraosseous in the area usually involved with sinus antrostomy (from the second premolar to the second molar) in $100 \%$ of cases; and (c) variable (either intraosseous or intrasinusal, or subperiosteal) in the maxillary tuberosity area. In the sinus antrostomy area, the alveolar antral artery was mostly located close to the Schneiderian membrane and partially encased in the lateral sinus wall in all specimens. No bony layer interposed between the alveolar antral artery and the sinus membrane could be identified by dissection. ${ }^{20}$ In the literature, 2 reports state that this vessel was in the first molar area, averaging $11.25 \pm 2.99 \mathrm{~mm}$ (range: $7.2-17.7 \mathrm{~mm}$ ). ${ }^{20}$ In the most atrophic cases, with a ridge 

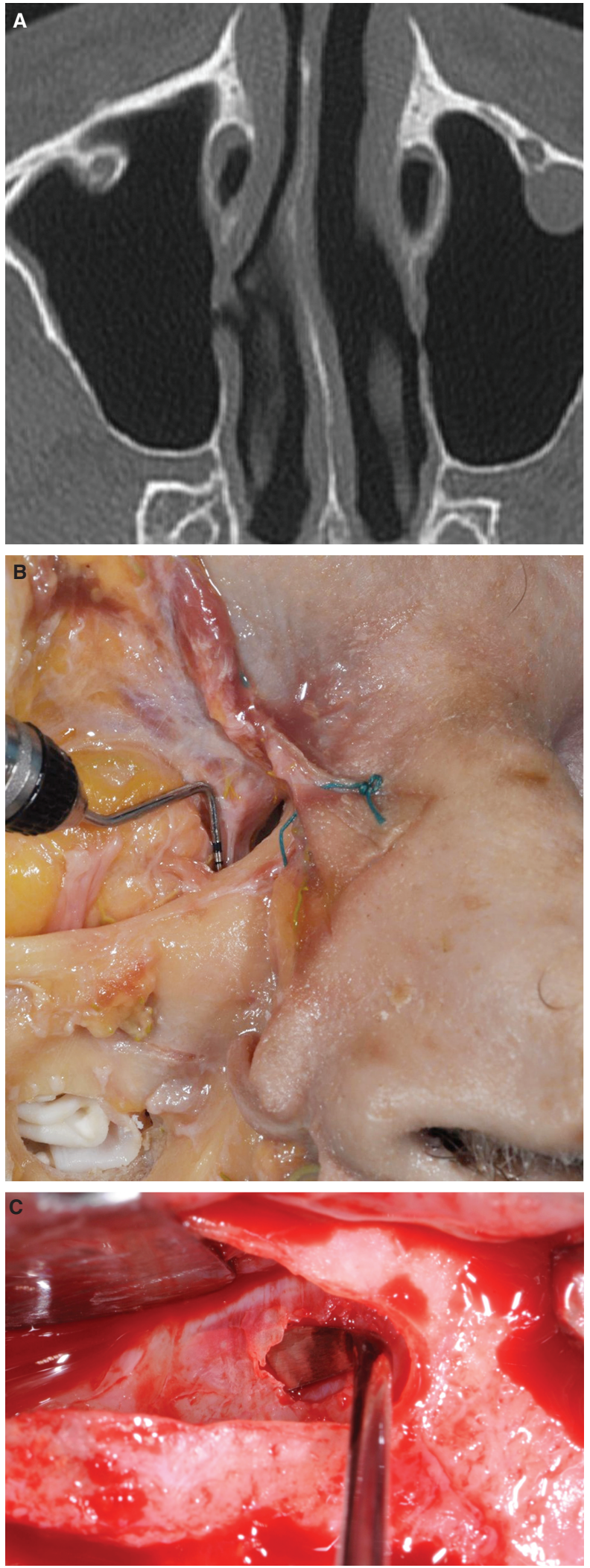

FIGURE 6 Nasolacrimal duct. (A) Radiographic view. (B) Cadaver dissection. (C) Clinical

height of $<3.0 \mathrm{~mm}$, the distance was significantly lower compared with cases that were less atrophic. This confirms that the more resorbed the bone crest, the higher the risk of violation of such a vessel during a sinus augmentation procedure. These results are substantially in agreement with a study by Mardinger et al, ${ }^{24}$ who found that this vessel was located at a mean distance of $10.9 \mathrm{~mm}$ from the crest in classes $D$ and $E$ and at a distance of $>15 \mathrm{~mm}$ in classes A, B, and C following the classification of Lekholm and Zarb. ${ }^{26}$ Moreover, a well-distinguished bony wall between the intraosseous maxillary anastomosis and the internal aspect of the maxillary sinus was never found by anatomic dissection in the cadaver study by Rosano et al, ${ }^{20}$ and therefore it could be assumed that the lowest border of such a vessel could often be completely adherent to the sinus membrane (ie, not radiographically visible) instead of being located inside the buccal wall cortex. This would justify the contradiction between a $100 \%$ prevalence of this artery found by dissection and a prevalence of only $47 \%$ detected by computed tomography in the study by Rosano et al. ${ }^{20}$ In the present author's opinion, such a contradiction may not be because of the small diameter of the alveolar antral artery but in fact is caused by the entirely intrasinusal location of the vessel that could not be viewed on cone-beam computed tomography scan because it is located outside the bony cavity of the sinus wall. The diameter of the anastomosis was $\geq 2 \mathrm{~mm}$ in only $3.3 \%$ of the cases analyzed by dissection and in only $2 \%$ of the cases analyzed by computed tomography. This possibility, even if infrequent, is worth taking into serious consideration. The transection of an alveolar antral artery with a diameter of $>2 \mathrm{~mm}$ is likely to result in bleeding and impairment of vision. The preservation of such an anastomosis may be important not only to avoid bleeding complications but also to support bone graft neoangiogenesis. ${ }^{20}$ In this perspective, its concomitant reflection with the Schneiderian membrane during sinus augmentation procedures should be considered if clinically feasible, especially when its diameter is large.

\subsection{Other anatomic features}

When elevating the membrane at the level of the nasal (medial) wall, care should be taken to respect the nasolacrimal duct as sometimes only a thin layer of bone (a few tens of millimeters) separates the nasolacrimal duct from the sinus (Figure $6 \mathrm{~A}-\mathrm{C}$ ).

Vertical releasing incisions should be full thickness in keratinized gingiva and split thickness in the alveolar mucosa to avoid neurologic injury of the infraorbital nerve. An accessory ostium can allow an endoscope to be inserted into the sinus during maxillary sinus elevation for preoperative ear, nose, and throat evaluation because the natural ostium is not suitable for intrasinus endoscopic examination (Table 1).

\section{3 | PRESURGICAL SINUS ASSESSMENT: DIAGNOSING PATHOLOGIC CONDITIONS OF THE MAXILLARY SINUS}

It can be stated that the clinician is able to lower the risk of preoperative and postoperative complications if maxillary sinus elevation 
TAB LE 1 Maxillary sinus anatomy and its clinical implications

\begin{tabular}{|c|c|}
\hline Sinus anatomy & Clinical implications \\
\hline $\begin{array}{l}\text { Angle made by the buccal and } \\
\text { palatal alveolus at crest }\end{array}$ & Angle $\mathrm{a}<30^{\circ}$ (perforation rate $62.5 \%$ ) Angle $\mathrm{a}<30^{\circ}>60^{\circ}$ (perforation rate $28.6 \%$ ) \\
\hline Vascularity & $\begin{array}{l}\text { Preservation of vascularity may be important not only to avoid bleeding complications but also to support } \\
\text { bone graft neoangiogenesis }\end{array}$ \\
\hline Naso-lacrimal duct & $\begin{array}{l}\text { Do not be aggressive when elevating the membrane at the level of the nasal wall: a thin layer of bone (pos- } \\
\text { sibly only a few tens of millimeters) may separate the nasolacrimal duct from the sinus (Figure } 6 \mathrm{~A}-\mathrm{C} \text { ) }\end{array}$ \\
\hline Infraorbital nerve & $\begin{array}{l}\text { Full-thickness vertical releasing incisions in the premolar area can cause neurological disturbances to the } \\
\text { branches of infraorbital nerve }\end{array}$ \\
\hline
\end{tabular}

is performed starting from a healthy sinus with high compliance. ${ }^{27,28}$ It is therefore advisable to perform extensive anamnestic, clinical, and radiographic assessments before sinus augmentation surgery in order to investigate sinus health and subsequent sinus compliance, with the aim of avoiding postsurgical complications. It is extremely important during the first consultation to collect a complete history of potential diseases affecting the maxillary sinus, such as nasal obstructions, facial trauma, sinus infections, allergic symptoms, smell and taste dysfunction, pressure-related discomfort, chronic respiratory diseases, previous nasosinusal surgery, facial deformities, scars, and mouth breathing. If the anamnesis is positive or there are symptoms of sinusitis, it is advisable to ask for an ear, nose, and throat assessment. The same assessment should be made in the case of radiologic signs of radio-opacity, previous sinus treatments, impaired nasal breathing, and chronic respiratory diseases. Table 2 proposes a list of questions for a specific maxillary sinus anamnesis.

Cone-beam computed tomography is considered as the fundamental tool for evaluating the anatomy and health of the maxillary sinus. The cone-beam computed tomography analysis should be extended superiorly to include the osteomeatal complex, in order to assess the patency of the ostium. This is a broader field of view than is traditionally obtained for routine maxillary implant placement. Thickening of the mucosa is usually a sign of altered sinus physiology. Carmeli et al $^{29}$ evaluated 560 maxillary sinuses through computed tomography scans and classified different grades of mucosal thickening. A rounded mucosa is usually associated with a low risk for a future maxillary sinus elevation, while irregular, circumferential, and/or complete thickening is associated with an increased risk for sinus obstruction. Another common finding is the presence of mucous retention cysts. Although their pathogenesis is not completely clear, there is some agreement of the theory that mucous retention cysts develop as a result of obstruction of the ducts of the mucous-producing glands. ${ }^{30}$ The cyst wall is the ductal epithelium and capsule of the gland. ${ }^{31}$ Another possibility occurs when serous fluid accumulates in the submucosal layer of the sinus and a serous retention cyst is developed. The mucosa of the sinus then becomes the wall of the cyst. ${ }^{30}$

When the lamina propria of the sinus membrane is affected by inflammation and edema, sinus polyps may occur. ${ }^{30}$ They have a solid consistency and even though it is very difficult to differentiate them from a mucous retention cyst, their treatment is the same. ${ }^{30}$ A high air-fluid level usually results in bacterial sinusitis and, from a radiological point of view, is a straight line or meniscus. ${ }^{30}$ When the sinus is completely opacified and the sinus cavity is enlarged, this is diagnosed as a mucocele. ${ }^{30}$ Fortunately, this occurs very rarely in the maxillary sinus. ${ }^{32}$ Pignataro et $\mathrm{al}^{33}$ present a series of clinical recommendations concerning ear, nose, and throat contraindications to maxillary sinus elevation (Table 3 ).

Mantovani ${ }^{34}$ have divided contraindications for maxillary sinus augmentation into potentially reversible and presumably irreversible categories (Table 4).

A prospective clinical study evaluated this approach and confirmed its reliability. ${ }^{16}$ Thirty-four patients were evaluated. None presented presumably irreversible contraindications, but $38.2 \%$ presented potentially reversible contraindications and were consequently treated, and no complications after sinus lift surgery were identified (refer to Table 4).

\section{1 | Odontogenic sinusitis maxillaris}

A sinus lift procedure can be impaired by a preexisting odontogenic sinusitis. Odontogenic sinusitis represents $10 \%$ of all cases of maxillary sinusitis ${ }^{35,36}$ but it is estimated that the real incidence could be between $25 \%$ and $40 \%{ }^{37,38}$ A survey ${ }^{39}$ by 93 board-certified otolaryngologists and rhinologists reports that an odontogenic source is a common cause of maxillary sinusitis and reported treating an average of 2.9 patients per year with odontogenic maxillary sinusitis, who were initially misdiagnosed. Otolaryngologists also perceived that radiologists rarely consider dental pathology when scanning the maxillary sinus using computed tomography. The exact 
TABLE 2 Maxillary sinus medical history

\begin{tabular}{l} 
Medical history \\
\hline Do you suffer from any kind of allergy?
\end{tabular}

Does the computed tomography allow a correct visualization of the osteomeatal complex?

Is the osteomeatal complex patent?

Are there any signs of radio-opacity in the maxillary sinus?

Final evaluation

Ask for an ear, nose, and throat assessment

Patient eligible for maxillary sinus elevation

TABLE 3 Ear, nose, and throat assessment

\section{Ear, nose, and throat assessment of candidates for maxillary sinus lift procedure}

1. Preventive-diagnostic step aimed at excluding any nasosinusal diseases that may lead to failure of surgery

2. Preventive-therapeutic step aimed at correcting any pathologic findings that represent reversible contraindications to a sinus lift

3. Diagnostic-therapeutic step (if necessary) aimed at ensuring the prompt diagnosis and appropriate treatment of any possible sinus lift-related nasosinusal complications

pathogenesis of odontogenic sinusitis is still not fully understood, although impaired Schneiderian membrane integrity as a result of maxillary dental infections or trauma, odontogenic disease of maxillary bone, tooth extractions, implantology, or endodontic treatment is always present. Microbiologic sampling of sinusitis of odontogenic origin reveals a different bacterial flora than that found in rhinogenic sinusitis. ${ }^{40}$ Usually odontogenic sinusitis is a polymicrobial infection, and anaerobic species from the oral cavity and upper respiratory tract are predominant. The development of sinusitis in patients with predisposing odontogenic disease is variable, but a recent review suggests a possible role of the bacterial biofilm in the severity and progression of odontogenic sinusitis. ${ }^{41}$ Bacterial biofilm, defined as dynamic polymicrobial communities of slowly replicating and metabolically quiescent strains embedded in a matrix rich in exopolysaccharides, proteins, and nucleic acid, ${ }^{42}$ is associated with many endodontic lesions linked to odontogenic sinusitis (Table 5). ${ }^{43}$

Although no specific studies of odontogenic sinusitis have yet been carried out, the findings of animal studies and clinical trials involving patients with chronic rhino-sinusitis suggest a link between a polymicrobial bacterial biofilm (mainly composed of Staphylococcus aureus, Pseudomonas aeruginosa, coagulase-negative staphylococci, Streptococcus pneumoniae, Moraxella catarrhalis, Haemophilus influenzae, and fungal species) and recurrent or recalcitrant paranasal sinus infections. ${ }^{44-48}$ Pathogens mainly involved in bacterial biofilmrelated chronic rhino-sinusitis are $S$. aureus, $H$. influenzae, P. aeruginosa and S. pneumonia, ${ }^{49,50}$ as well as anaerobic species. Particular interest has recently been aroused by the discovery of specific S. aureus-producing toxins that are known to lead to chronic inflammation in patients with chronic rhino-sinusitis and nasal polyps. In the presence of persistent staphylococcal infection sustained by biofilm, toxin production might also occur in the absence of planktonic species, thus leading to chronic immune system activation and persistent inflammation. ${ }^{51}$ These findings are extremely important, especially when choosing the correct antibiotic therapy for treating sinusitis. 
TAB LE 4 Contraindications for maxillary sinus augmentation

Presumably irreversible ear, nose, and throat contraindications

Anatomic-structural alterations: Serious deformities and posttraumatic, postsurgical, and postradiotherapy scarring on the nasalsinus walls and/or mucosa lining
Inflammatory-infective processes: Reoccurring or chronic sinusitis, with or without polyps, which cannot undergo resolution as it is associated with congenital mucociliary clearance alterations (eg, cystic fibrosis, Kartagener's syndrome, Young's syndrome), intolerance of acetylsalicylic acid (triad: nasal polyps, asthma, intolerance to acetylsalicylic acid), or immunologic deficiency (eg, AIDS, pharmacologic immuno-suppression)

Nasal-sinus manifestations of aspecific systemic granulomatous diseases: Wegener's granulomatosis, "idiopathic midline granuloma," and sarcoidosis

Tumor-related: Locally aggressive benign tumors (eg, inverted papilloma, myxoma, ethmoidal- maxillary fibromatosis) in antrum; Nasal-sinus malignant tumors (epithelium, neuroectodermal, bone, soft tissue, odontogenous, lymphomatosis, metastatic-originated) of the maxillary sinus and/or adjacent structures
Potentially reversible ear, nose, and throat contraindications

Anatomic-structural alterations: Stenosis of the drainage-ventilation pathways in the maxillary sinus (sustained by one or more of the following anatomic alterations): septal deviation, paradox curve of the middle turbinate bone, conchae bulla, hypertrophy of the agger nasi cell, presence of Haller cell), postsurgical scars or synechiae on the osteomeatal complex, oroantral fistula. All these alterations can be resolved by surgery: the maxillary sinus appears to be well ventilated as a result of a partial uncinectomy

Inflammatory-infective processes: Acute viral or bacterial rhinosinusitis, allergy-related rhinosinusitis, mycotic sinusitis (non-invasive forms), acute repeating and chronic sinusitis sustained by 1 of the anatomic alterations listed above which obstruct the sinus drainage-ventilation pathways, by endo-antral foreign bodies, or by nasal polyps. Functional endoscopic surgery is clearly indicated

Tumor-related: Nonobstructive nasal-sinus benign tumors, both before and after the lifting operation, could affect the sinus drainage-ventilation pathways or when removal does not affect the mucociliary transportation system (eg, mucosal cysts, cholesterinic granuloma, antrochoanal polyp); all are easily subject to correction by functional endoscopic surgery

Note: (Modified from Mantovani M (ed.). ${ }^{34}$

A retrospective evaluation ${ }^{52}$ reviewed 174 patients treated surgically for unilateral symptomatic maxillary sinusitis over 7 years. In $75 \%$, the sinusitis was triggered by odontogenic pathology, and dentoalveolar surgical interventions were the most common (64\%) cause. These results suggest that when there is a unilateral maxillary sinusitis an odontogenic cause is very likely. Although understanding the microbiology of the lesion will provide a great opportunity to treat the sinusitis in the best possible way, it is always advisable to refer the patients in such cases to ear, nose, and throat specialists should resolution not be rapidly achieved.

\section{4 | BEHAVIOURAL CONDITIONS IMPAIRING MAXILLARY SINUS HEALTH STATUS}

Sinus lift procedures could be affected by a number of behavioral and environmental conditions affecting the normal physiology of the maxillary sinus. The use of cocaine, a drug usually inhaled through the nose, has a dramatic effect on the oral mucosa (Figure 7). ${ }^{53}$

In a systematic review addressing hard palate perforation in cocaine abusers, sinusitis is confirmed as one of the most common side effects. ${ }^{54}$ From a clinical point of view, in the authors' experience, the Schneiderian membrane in these patients appears extremely thin and fragile, requiring close attention when detaching. Smoking is a well-known risk factor for implant survival. ${ }^{55} \mathrm{~A}$ retrospective evaluation on the survival rate of implants placed in grafted sinuses found that smoking more than 15 cigarettes per day was significantly correlated to implant failure. ${ }^{56}$

\section{5 | PREVENTION AND TREATMENT OF SURGICAL COMPLICATIONS}

\section{1 | Intraoperative complications}

Any list of potential intraoperative complications will be quite extensive given the broad scope of this surgical procedure. It is therefore important to understand that the relative frequency of the majority of these complications is quite low. Most intraoperative complications are primarily caused by surgical difficulties encountered during the course of the augmentation procedure. These may be a result of the presence of complex anatomic situations (thin membranes; incomplete, thick, or convex lateral walls; septa; presence of cysts), the choice of less predictable treatment options, inadequate preoperative systemic and/or local anatomic diagnosis, or operator error.

The most common intraoperative complication is Schneiderian membrane perforation. Other, less common, complications include intraoperative bleeding, perforation of the buccal flap and (much less frequently) injury to the infraorbital nerve, damage to the adjacent dentition, perforation of medial or the orbital wall, implant displacement into the maxillary or paranasal sinuses, and obstruction of the ostium. 
TAB LE 5 Bacterial species linked to odontogenic sinusitis ${ }^{84}$

\begin{tabular}{|ll|}
\hline Bacterial species & \\
\hline Actinomyces israelii & Clostridium sordellii \\
\hline Actinomyces viscosus & Clostridium bifermentans \\
\hline Actinomyces meyeri & Staphylococcus chromogenes \\
\hline Actinomyces naeslundii & Staphylococcus epidermidis \\
\hline Propionibacterium acnes & Streptococcus spp. \\
\hline Propionibacterium propionicum & \\
\hline Peptostreptococcus prevotii & \\
\hline Gemella morbillorum
\end{tabular}

\section{2 | Intraoperative bleeding}

\subsection{1 | Etiology and incidence}

Intraoperative bleeding results from severing or damaging branches of the vascular supply to the lateral wall of the sinus and the surrounding soft tissues. This bleeding is usually minor and of relatively short duration, but in some instances it can be profuse and difficult to control. Solar et $\mathrm{al}^{21}$ described the blood supply to the lateral wall of the maxillary sinus in cadaver specimens. Blood is supplied through the intraosseous and extraosseous branches of the posterior superior alveolar artery, which form a double arterial arcade by anastomosing with the infraorbital artery. Bleeding may occur either from the soft tissue (the extraosseous branch) during flap elevation or directly from the lateral bony wall (the intraosseous branch) during preparation of the lateral window via rotary instrumentation. There is also the possibility of bleeding from the medial wall of the sinus if the posterior lateral nasal artery is damaged. ${ }^{57}$ The posterior superior alveolar, infraorbital, and posterior lateral nasal arteries are all branches of the maxillary artery that provides a source of blood for vascularization of the sinus graft.

\subsection{2 | Prevention}

Although bleeding does not occur on every occasion that this artery is damaged, it seems prudent to use 3-dimensional planning as a means of avoiding, if possible, an encounter with the artery (Figure 8). In some cases, the artery can be visualized within the lateral wall after elevation of the flap (Figure 9). In many instances, a window can be made coronal to the location of the artery and the superior portion of the membrane elevation can be performed internally to the required height. Again, it should be recognized that the artery is not always located within the lateral wall. The artery can be located just internal to the lateral wall and may pass in and out of the bony wall throughout its antero-posterior course in the lateral sinus wall. When located outside the lateral wall, it is susceptible to damage from both rotary and hand instruments. The external branch of the posterior superior alveolar artery may also be damaged when making vertical releasing incisions for flap elevation.

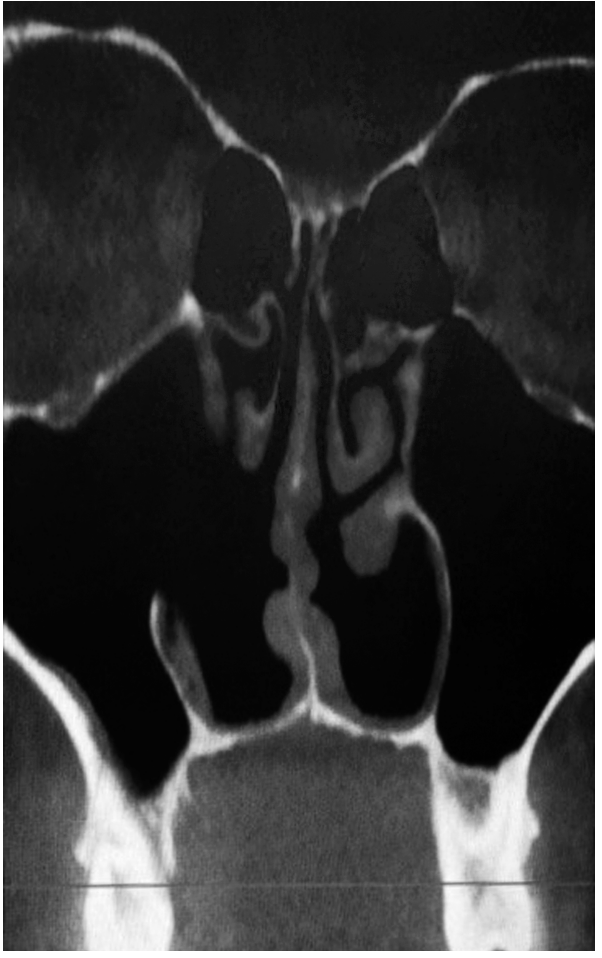

FIGURE 7 Cone-beam computed tomography showing the effects of cocaine use on the maxillary sinus

Once it is anticipated that the possibility of a bleeding complication exists, it is prudent to locate the position of the artery on the cross-sectional computed tomography images and then use antrostomy instruments that can respect the integrity of vascular and other soft tissues while still creating the window in the ideal location for access to and elevation of the sinus membrane. If rotary instruments are used, diamond burs are preferable to carbide burs as they are less likely to catch and tear the membrane. Piezosurgery ${ }^{\circledR}$, a concept of ultrasonic bone surgery developed by Vercellotti and specifically adapted for sinus elevation surgery, ${ }^{58}$ provides a means of avoiding this complication almost entirely. Piezoelectric surgery uses low-frequency ultrasonic vibrations $(24-32 \mathrm{kHz}$ for the various commercial systems) to perform cutting (osteotomy) and grinding (osteoplasty) procedures on bone. This low-frequency selective cutting action is safe for soft tissues, as it is incapable, if used correctly, of cutting blood vessels or the Schneiderian membrane. Piezoelectric surgery has been used successfully to avoid soft-tissue complications (both vascular and neural) in numerous oral surgical procedures, such as Le Fort osteotomies ${ }^{59}$ and mandibular sagittal split osteotomies. ${ }^{60}$ The Piezosurgery ${ }^{\circledR}$ technique has seen widespread use in Europe for over 17 years, and at the time of writing at least 6 piezoelectric surgery devices are available commercially in the USA. Since introduction of this technique to the USA in 2005, numerous clinicians have become aware of its advantages in sinus elevation surgery. Piezoelectric surgery has minimized bleeding episodes during preparation of the lateral window. The selective cutting action (in which only bone is cut) allows the operator to dissect the posterior superior alveolar artery from the bony window area, leaving it completely 


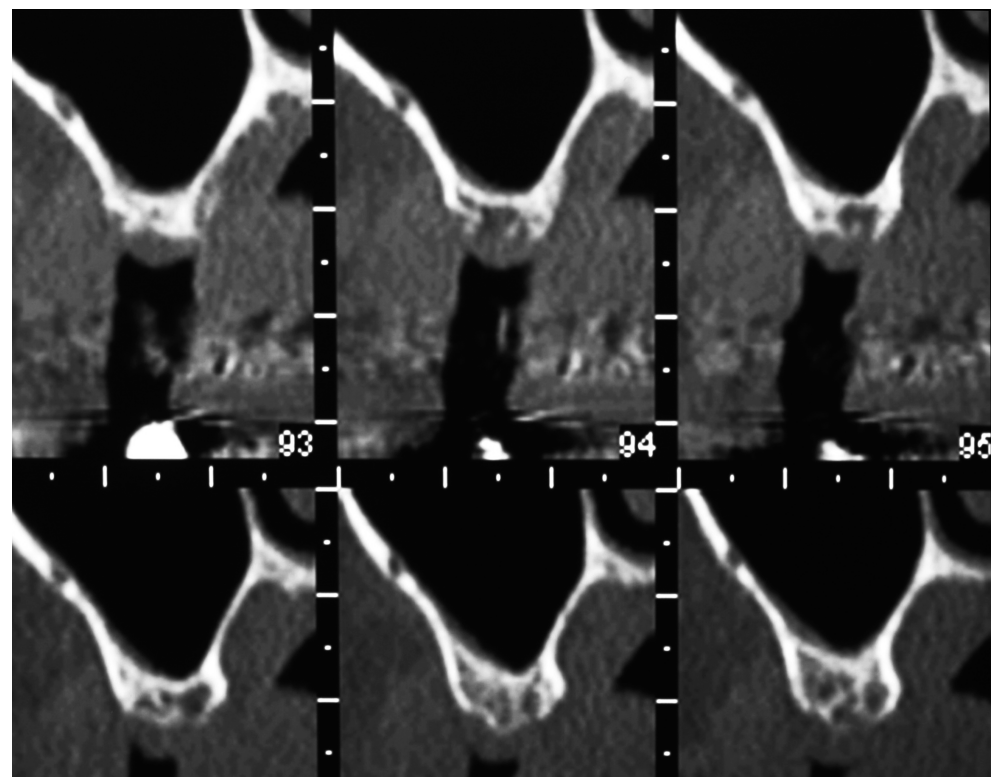

FIGURE 8 Posterior superior alveolar artery visualized in cross-sectional (paraxial) images of the lateral wall

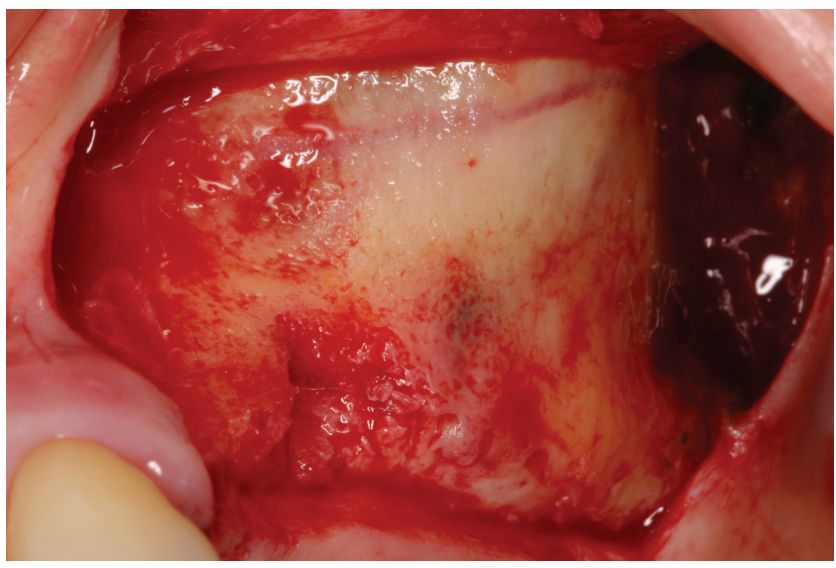

FIGURE 9 Artery visualized in the lateral wall after flap reflection

intact. Piezoelectric surgery has minimized bleeding episodes during preparation of the lateral window for most operators, including the authors. A recent systematic review showing comparable results regarding membrane perforations for both rotary and piezoelectric techniques is based on only 4 studies ( 1 favoring the rotary technique, 1 favoring the piezoelectric technique, and 2 with comparable results) with operator experience being a greater factor in the results than the actual surgical technique. ${ }^{61}$

\subsection{3 | Treatment}

Many techniques exist to control vascular bleeding in sinus elevation surgery. These include:

- direct pressure on the bleeding point.

- use of a localized vasoconstrictor.

- bone wax.

- crushing the bone channel around the vessel (hemostat).
- use of electrocautery (with care near membranes).

- suturing the vessel proximal to the bleeding point.

The use of a vasoconstrictor (1:50 000 epinephrine) is more effective than electrocautery in controlling soft tissue bleeding that may occur when making releasing incisions before elevation of the mucoperiosteal flap, while electrocautery is more effective than a vasoconstrictor in controlling a bone bleed from the cut lateral wall. It should be borne in mind that electrocautery, when used to control vascular bleeding from bone in the vicinity of the Schneiderian membrane, may result in membrane damage and therefore should be used with caution. Crushing the bleeding end of an intrabony vascular channel to compress the bone and vessel may be effective, but again care must be taken to avoid membrane perforation by direct pressure.

Surgeons can avoid membrane perforation by careful release of the Schneiderian membrane immediately internal to the vessel (making the window slightly larger, while diverting the blood flow with a suction tip to provide better vision) and then clamping the vessel with a hemostat.

Bleeding encountered during sinus elevation will usually be gently flowing in nature. In some instances, however, this bleeding may be pulsating. In general, the appearance is worse than the severity of the condition. Bleeding, even of the pulsating variety, may stop spontaneously or after several minutes of direct pressure as a result of clot formation within the bone channel surrounding the artery. One technique that may be used is to have the surgical assistant place a high-volume, narrow-tipped evacuator close to the bleeding point to eliminate blood flow into the surgical field. Window preparation, membrane elevation, and grafting can be completed while diverting bleeding in this manner. The bleeding usually stops by the time the grafting is completed and, after closure, postoperative bleeding is usually not encountered. Note that suction is utilized only to create adequate vision. It is detrimental to halting the bleeding.

Best clinical practice includes: 
- obtain preoperative computed tomography images to locate the vessel.

- visualize the vessel clinically.

- avoid the vessel, if appropriate, when designing the window.

- use piezoelectric surgery to avoid trauma to the vessel.

- have materials on hand to control bleeding (electrocautery, local with 1:50 000 epinephrine, bone wax, resorbable suture material).

\section{3 | Schneiderian membrane perforation}

\subsection{1 | Etiology and incidence}

Perforation of the Schneiderian membrane is the most common intraoperative complication in sinus elevation surgery. ${ }^{61,62}$ The reported incidence in the literature varies from $11 \%^{63}$ to $56 \%{ }^{64}$ when rotary window preparation is used. Most experienced clinicians estimate their perforation rate to be approximately $25 \%$ when using conventional rotary instruments. In retrospective computed tomography studies performed at the New York University Department of Periodontology and Implant Dentistry (Poster Presentation, AO Annual Meeting, 2002), the perforation rate was shown to have a close relationship to membrane thickness and, to a lesser degree, to the presence of septa. The perforation rate was $41 \%$ when the membrane thickness was $<1.5 \mathrm{~mm}$ and $16.6 \%$ when it was $\geq 1.5 \mathrm{~mm}$. The perforation rate in a separate study of 136 sinus elevation procedures was $44.2 \%$ when a septum was present and $35.7 \%$ when septa were absent. In a retrospective computed tomography study by Cho et al, ${ }^{65}$ the perforation rate was shown to be related to sinus width or, to be more specific, the angle made by the medial and lateral walls at the floor of the sinus. The perforation rates were $62.5 \%$ for the narrow anterior part of the sinus (angle $<30^{\circ}$ ), $28.6 \%$ for the wider middle part of the sinus (angle $30-60^{\circ}$ ), and $0 \%$ for the widest posterior portion (angle $>60^{\circ}$ ) (Figure 10). A recent computed tomography study by Chan et $a^{66}$ identified another "angle," which defines the various configurations of the palatonasal recess and must be taken into consideration when elevating the Schneiderian membrane from the medial wall (Figure 11). It is the angle made where the alveolus meets the medial wall of the sinus. If this angle is acute, and is located within approximately $10 \mathrm{~mm}$ from the sinus floor (an area where graft material is likely to be placed), care must be taken to keep the elevator on the bone surface while not trapping, and thus tearing, the membrane.

There are numerous maneuvers that must be performed during sinus elevation surgery which may place the Schneiderian membrane at risk. These include:

- flap elevation (placing an elevator through a thin crest or lateral wall or through a previous oroantral fistula that has healed with soft tissue only).

- preparation of the lateral window (specifically with rotary instruments).
- elevation of the Schneiderian membrane with hand instruments (narrow sinus, acute angles, thin membrane, and in close proximity to septa).

- placement of graft material (excessive pressure against membrane).

\subsection{2 | Prevention}

A thorough knowledge of the 3-dimensional anatomy of the sinus is essential if the perforation rate is to be kept to a minimum. A computed tomography analysis will provide information relating to the thickness of the crest and lateral walls, the presence of discontinuities in the bony walls, the width of the sinus, the slope of the anterior sinus wall, membrane thickness, and the presence, size, and location of septa. Clinicians will also gain information relative to sinus health and patency of the osteomeatal complex. This evaluation may indicate the need for presurgical treatment that can avoid complications, such as postoperative sinusitis and infection. Figure $12 \mathrm{~A}, \mathrm{~B}$ shows a defect in the lateral sinus wall created during a failed sinus elevation. Likewise, lateral wall defects may be created during extraction of teeth. It is possible that an aggressive flap elevation procedure may cause a tear in the membrane at this location. If a discontinuity is known to exist, a split-thickness flap dissection over the site will avoid laceration of the sinus membrane. Having 3-dimensional knowledge of the existence, location, and anatomy of a septum will help determine the best location for the antrostomy in order to facilitate an uneventful membrane elevation.

A septum may initially be seen as a ridge crossing the sinus floor but it will generally continue as a spine, reaching its highest extent on the medial wall (Figure 13A-C). ${ }^{18}$

Septa can be quite large (Figure 14), but with proper access they can be circumvented. In rare cases the septum can be high enough to divide the sinus, at least at the working level, into 2 separate compartments (Figure 15).

Once inside the sinus, good access and good vision will greatly facilitate membrane elevation. The location of the lateral window and its size will affect the clinician's ability to elevate the membrane safely. Having the window in a location that gives the best access to areas where instrument angulation, and hence membrane elevation, is difficult will have a profound effect on the operator's ability to keep his or her hand instruments directly on the bone surface. Changes in instrument angulation are required to go across the floor and up the anterior and medial sinus walls. The anterior portion of the sinus can be very narrow, requiring coordination and visibility to prevent inadvertent membrane perforation. Many experienced clinicians consider that the ideal location for the window is $3 \mathrm{~mm}$ superior to the sinus floor and $3 \mathrm{~mm}$ distal to the sloping anterior wall, which allows controlled membrane elevation to be accomplished while keeping the elevating instruments on the bone surface at all times. Of the 11 perforations encountered by Zijderveld et al, ${ }^{62}$ were in relation to septa and 4 were made when releasing the membrane anteriorly with poor visibility. The superior extent of a sloping 


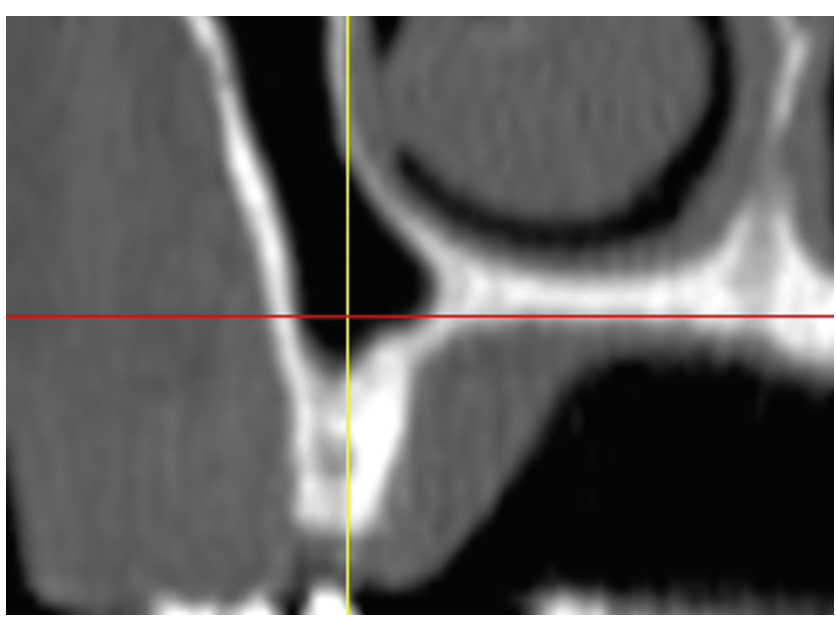

FIG URE 10 An acute "angle A" at the sinus floor of the anterior region

anterior wall may require the window to be far from a traditional oval or rectangular window. The shape of the window in this type of case should be trapezoidal, with the superior osteotomy cut being longer and more anterior than the inferior osteotomy, always keeping the window within $3 \mathrm{~mm}$ of the anterior wall. The anterior sinus wall should be considered an extension of the sinus floor, and the most predictable way to reach it during membrane reflection is by following the floor in an anterior and superior direction.

When septa are known to be present, it is advisable to lengthen the window in the antero-posterior direction so that the window is located both anterior and posterior to the septum. This allows for a lateral-to-medial elevation of the membrane from both sides of the septum. It must be recognized that it is extremely difficult to elevate a membrane from a sharp septum in a mesial-to-distal direction while keeping the elevator on the bony surface at all times. While making 2 separate windows has been proposed for this task, some explanation is required. It is likely that the 2 separate windows will be so decreased in size that access and vision will be made even more difficult. In practice, creating 1 large window with improved access to both sides of the septum may be a more practical solution. A useful technique is to perform a complete osteotomy, which entails removal of the lateral window by osteoplasty or careful lifting and removal of the bony window. This will readily reveal the location of a septum and allow its removal and subsequent membrane elevation from both sides. While enlarging the window will improve both access and visualization, it must be mentioned that a recent study by Avila-Ortiz et a ${ }^{67}$ has shown a significant inverse correlation between window size and vital bone formation. While this may be true, there is no evidence that the reported difference has clinical significance in regard to the outcome of implant survival.

An evolution in surgical protocols has resulted in 2 techniques for window preparation that most authors and clinicians have found to result in substantially decreased membrane-perforation rates. These techniques involve the utilization of piezoelectric surgical inserts or DASK ${ }^{\circledR}$ (Dentium Advanced Sinus Kit) drills.

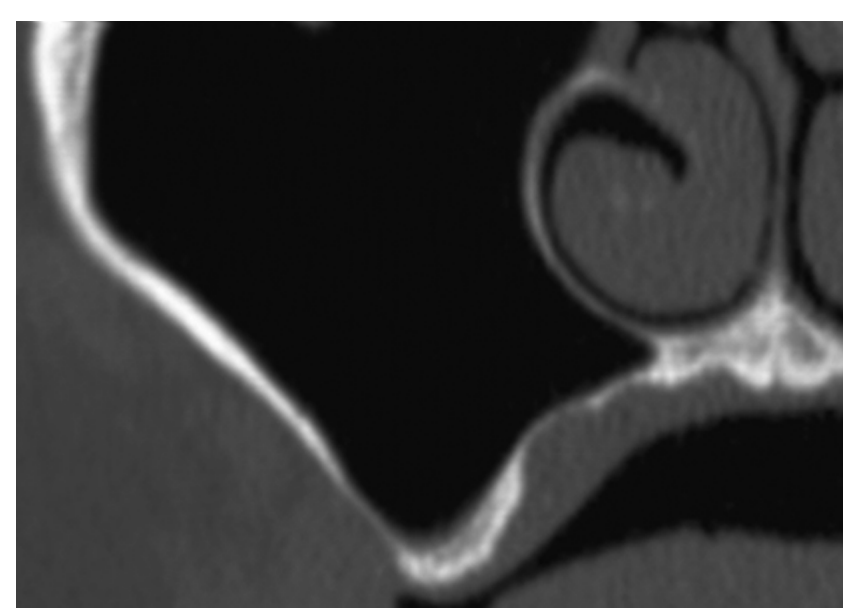

FIGURE 11 Acute angle in the palatonasal recess at the junction of the alveolus and the medial wall

Piezoelectric inserts have proven to be safe near soft tissue as a result of their engineered low-frequency ultrasonic vibration. In a series of 100 consecutive sinus elevation procedures using piezoelectric surgery, Wallace et al ${ }^{68}$ reported a membrane perforation rate of $7 \%$. In these series, all perforations occurred when completing the elevation using hand instruments, with no perforations occurring when the piezoelectric inserts were used. Blus et al $^{69}$ reported 2 perforations in 53 sinus elevation procedures (equivalent to a perforation rate of $3.8 \%$ ) using 2 different piezoelectric devices. In a report of 56 consecutive sinus elevation procedures, Toscano et al $^{70}$ reported a perforation rate of $3.6 \%$ using Piezoelectric surgery. Conflicting data were reported by Barone et al, ${ }^{70}$ who reported on 13 bilateral cases using Piezosurgery ${ }^{\circledR}$ on one side and a rotary diamond window preparation on the other as a within-patient control. The perforation rate was $30 \%$ with Piezosurgery ${ }^{\circledR}$ compared with $23 \%$ with the diamond bur control. Barone's results are contrary to the other publications and the positive clinical experience with piezoelectric sinus elevation surgery at both the New York University Department of Periodontology and Implant Dentistry and the Columbia University Division of Periodontics in the past 10 years.

Piezoelectric surgical techniques may differ depending upon the thickness and shape of the lateral sinus wall. If the window is thin, a diamond insert can be used to make a superior hinge or a free-floating bone island attached to the membrane (Figure 16A,B). This is then elevated horizontally. If the lateral wall is thick or it becomes convex in the malar eminence area, the entire lateral wall in the window area can be eliminated via osteoplasty (Figure 17A-C). You will then be looking directly at the Schneiderian membrane, which can be elevated with a combination of piezoelectric and manual elevators. Working directly against the membrane may seem to place the membrane at risk for perforation, but the membrane may be even more susceptible to damage from the sharp edges of an elevated bony window. While there is currently no histologic comparison of vital bone formation with these 2 techniques, clinical evidence from 
A
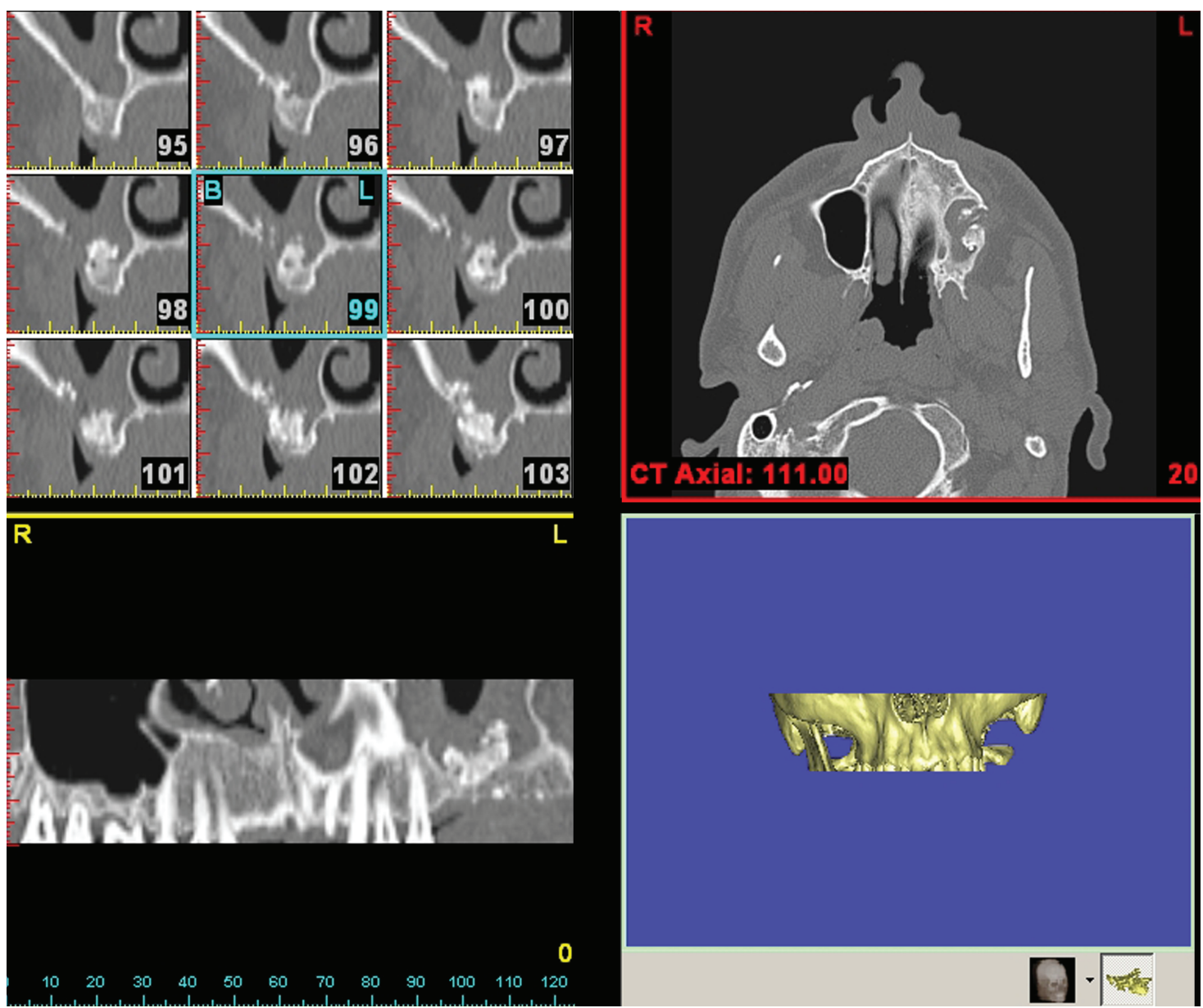

B

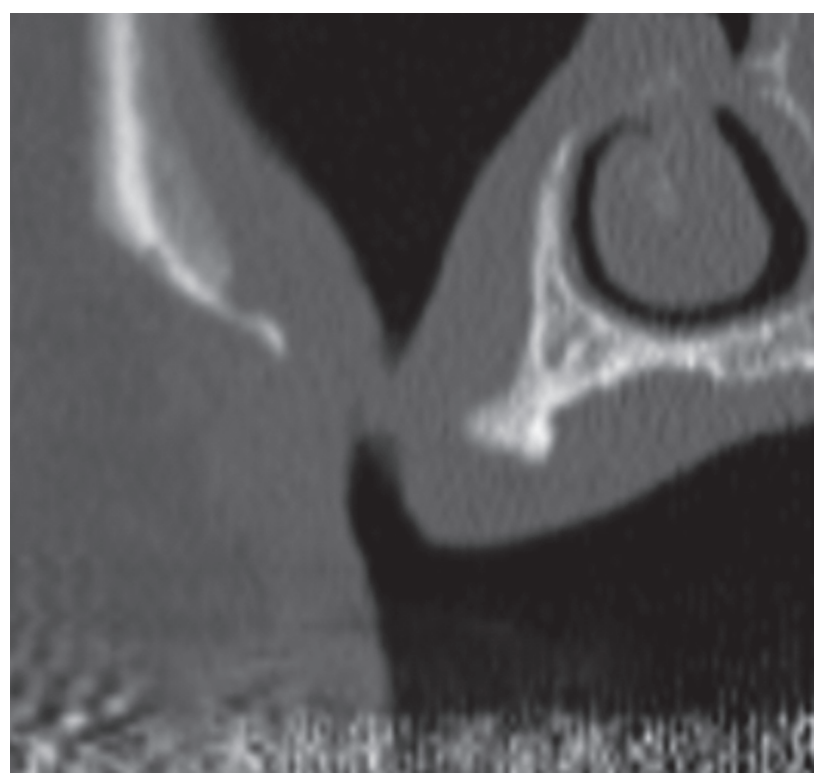

FIGURE 12 (A) Cross-sectional computed tomography-scan view showing defect in lateral wall. (B) Cross sectional computed tomography-scan view showing defect in lateral wall following extraction and healed oro antral fistula

the author's 14-year experience piezoelectric surgical technique does not show a difference in outcome, as measured by implant survival rate.
The DASK ${ }^{\circledR}$ technique utilizes a 6- or 8-mm-diameter domeshaped diamond drill to make the lateral window. The drill runs on a conventional implant motor at a speed of 800-1200 rpm with 


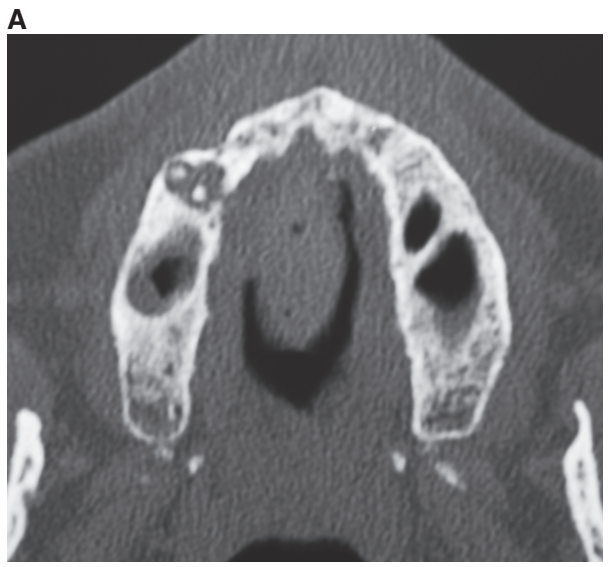

\section{B}

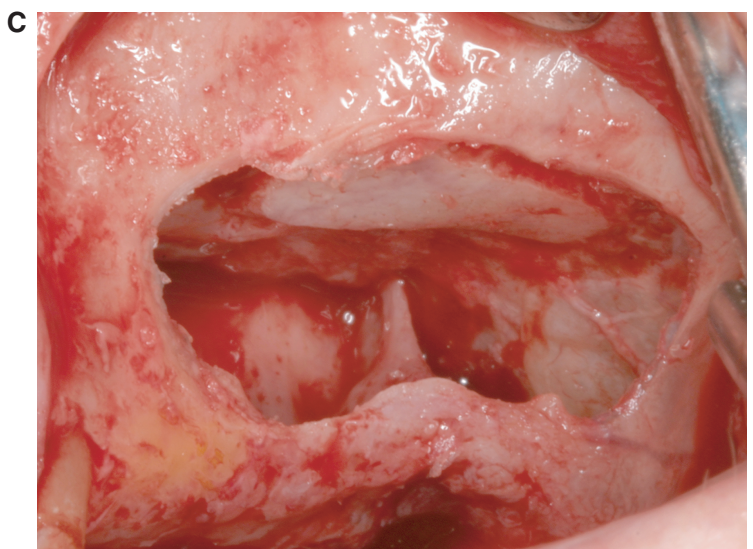

FIGURE 13 (A) Axial DentaScan view of a septum close to the sinus floor (left). (B) Axial DentaScan view of the same septum taken at a higher level. Note spine on left medial wall. (C) Clinical view of the septum shown in A and B

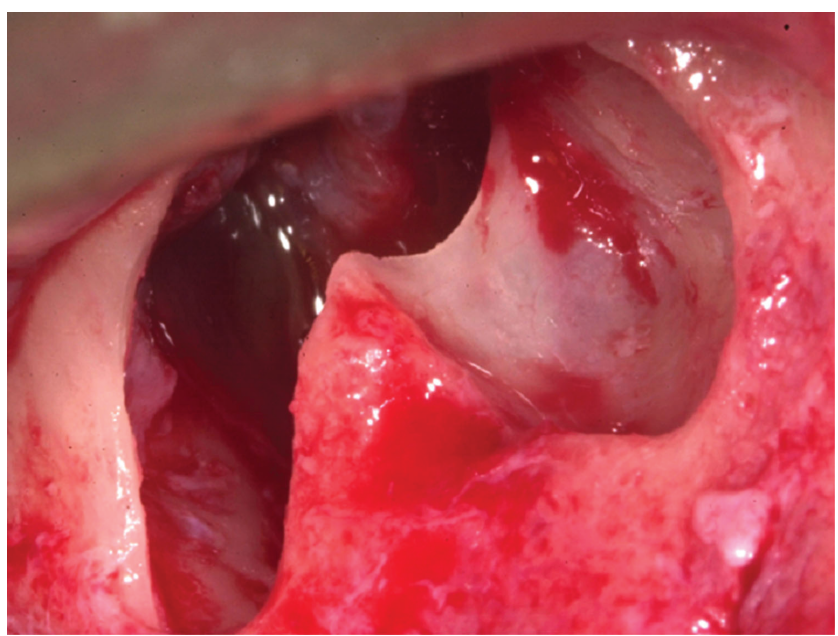

FIGURE 14 Exceptionally large septum

internal irrigation. The window can be made to have a round shape by utilizing an up-and-down movement, or it can be made to any size or shape desired by moving the drill in a lateral direction. This technique results in a complete osteotomy (total removal of window) in a safe manner as the large drill diameter and slow speed do not seem to cause the "drag" which is detrimental to membrane integrity. The drill appears to cut bone selectively, leaving the

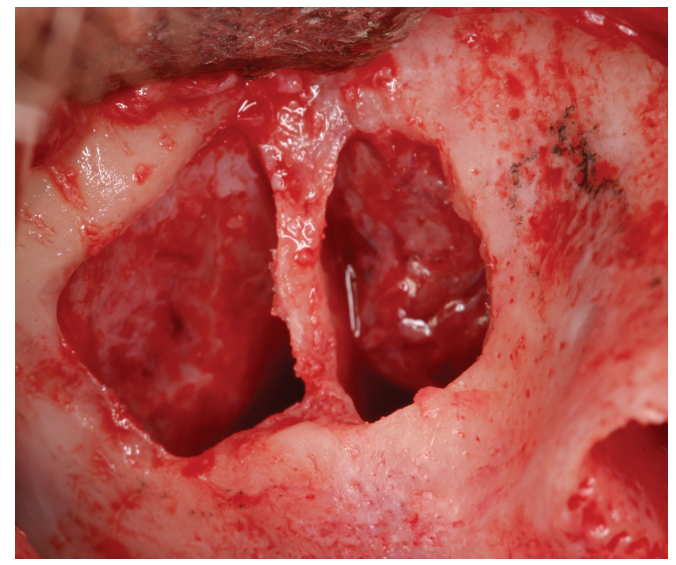

FIGURE 15 Septum dividing sinus into 2 compartments to a height of $18 \mathrm{~mm}$

exposed membrane intact. Membrane elevation then begins with either a motor-operated or hand-operated instrument that is similar in shape to the familiar "trumpet-shaped" piezoelectric elevator (Figure 18A-D). This technique, described as a lateral bone-planing antrostomy, has been shown, in a preliminary study by Lozada et $\mathrm{al}^{72}$ to result in a perforation rate of $5.6 \%$. An additional study by Nishimoto et $\mathrm{al}^{73}$ presented 50 consecutive cases with a perforation rate of $4 \%$. 

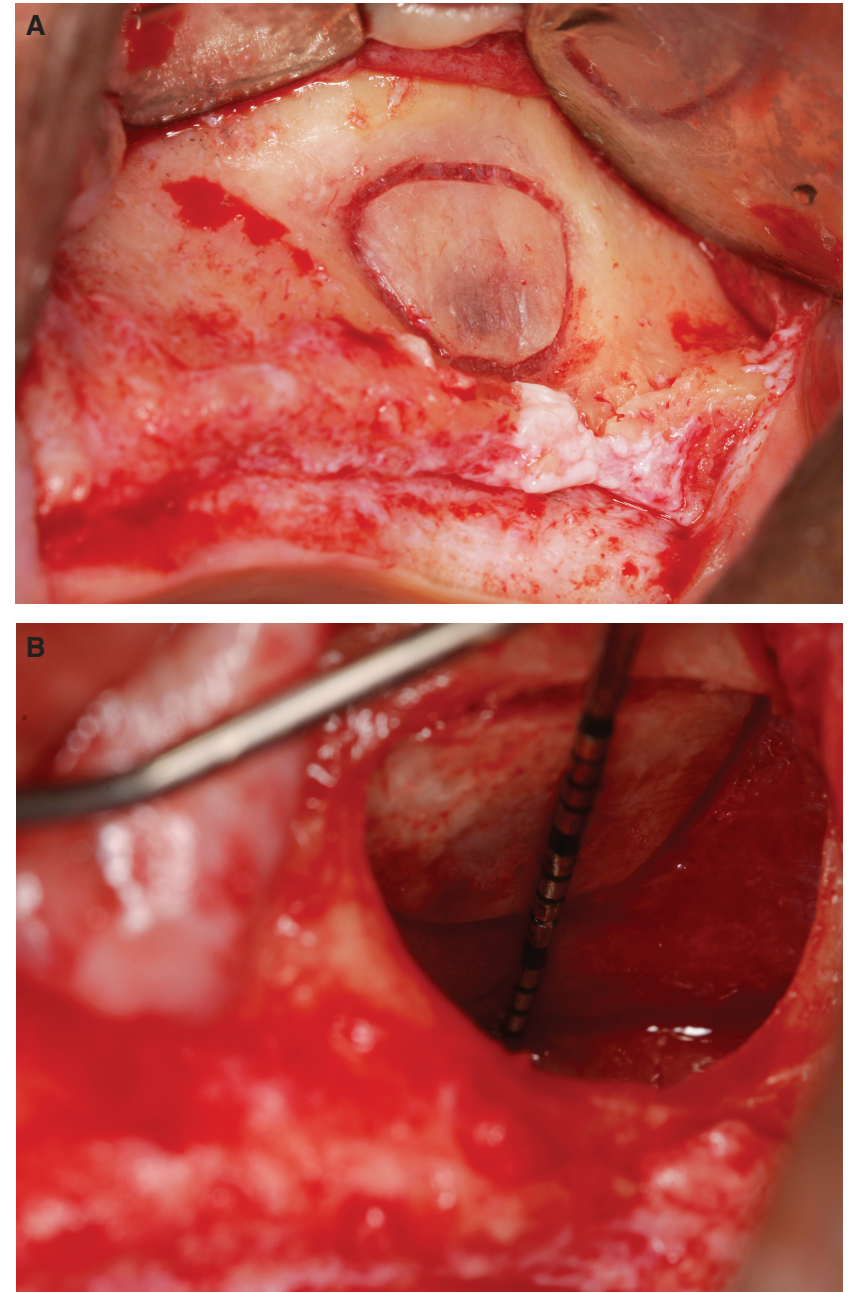

FIGURE 16 (A) Thin window outlined with a piezoelectric diamond insert. (B) Thin window with hinge or "island" has been elevated. Source: Wallace et al ${ }^{114}$ Reproduced with permission from Quintessence

\subsection{3 | Treatment}

An intact sinus membrane is essential for graft containment when using a particulate autogenous graft or particulate bone replacement graft as a sinus grafting material. This is not necessarily the case when using block grafts. ${ }^{74,75}$ Elevation of the Schneiderian membrane helps to form a compartment in which the particulate graft material can be placed and confined. The elevated membrane forms the distal and superior walls of this compartment, while the bony sinus walls form the inferior (crest), anterior, medial, and lateral walls. Proussaefs et $a^{76}$ showed that failure to contain the particulate graft as a result of membrane perforation will result in decreased bone formation (14.2\% vs $33.6 \%$ ) and a decreased implant survival rate (70\% vs $100 \%)$.

Should the sinus membrane be torn or perforated, the fragility of the remaining membrane becomes increased, and care and attention are required to complete the elevation. This is best accomplished by elevating the membrane around the perforation, thereby releasing tension on the perforated area of the membrane,
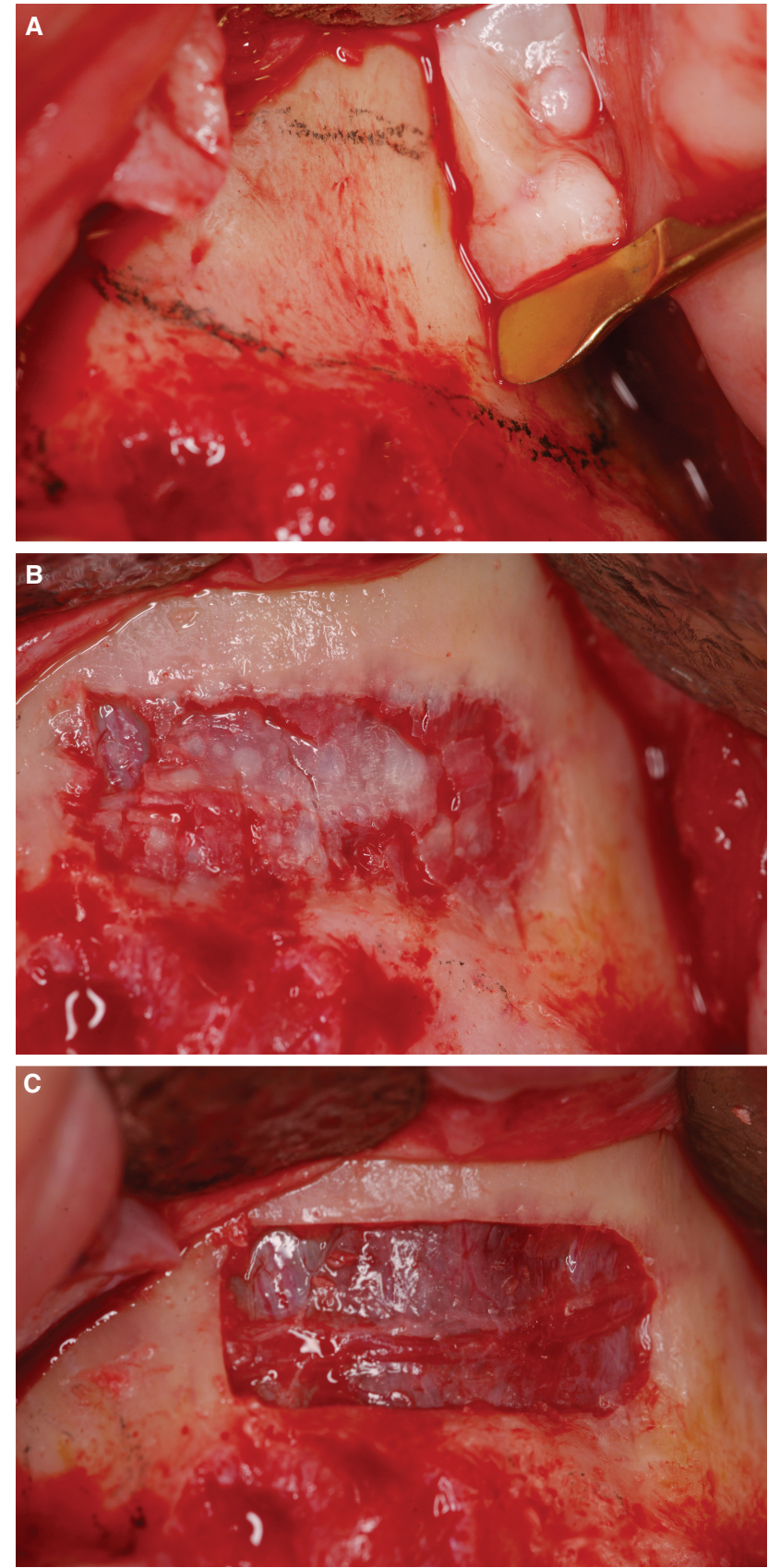

FIGURE 17 (A) Thick lateral wall with osteoplasty insert in place. (B) Lateral wall reduced to fragments by piezoelectric osteoplasty. (C) Complete osteotomy sparing vasculature

as opposed to working directly in the weakened area of the perforation. It is still necessary to complete elevation of the sinus membrane from the floor, medial, and anterior bony walls to allow the blood supply from the bony walls to vascularize the graft. Some clinicians prefer to make a small repair to stabilize the damaged area before completing the elevation. If this is done, the repair should be evaluated for stability before placing the graft material.

The most common means of repairing a perforated Schneiderian membrane is to use a bioabsorbable collagen barrier membrane as a patch. Other techniques involve the use of lamellar bone sheets, 

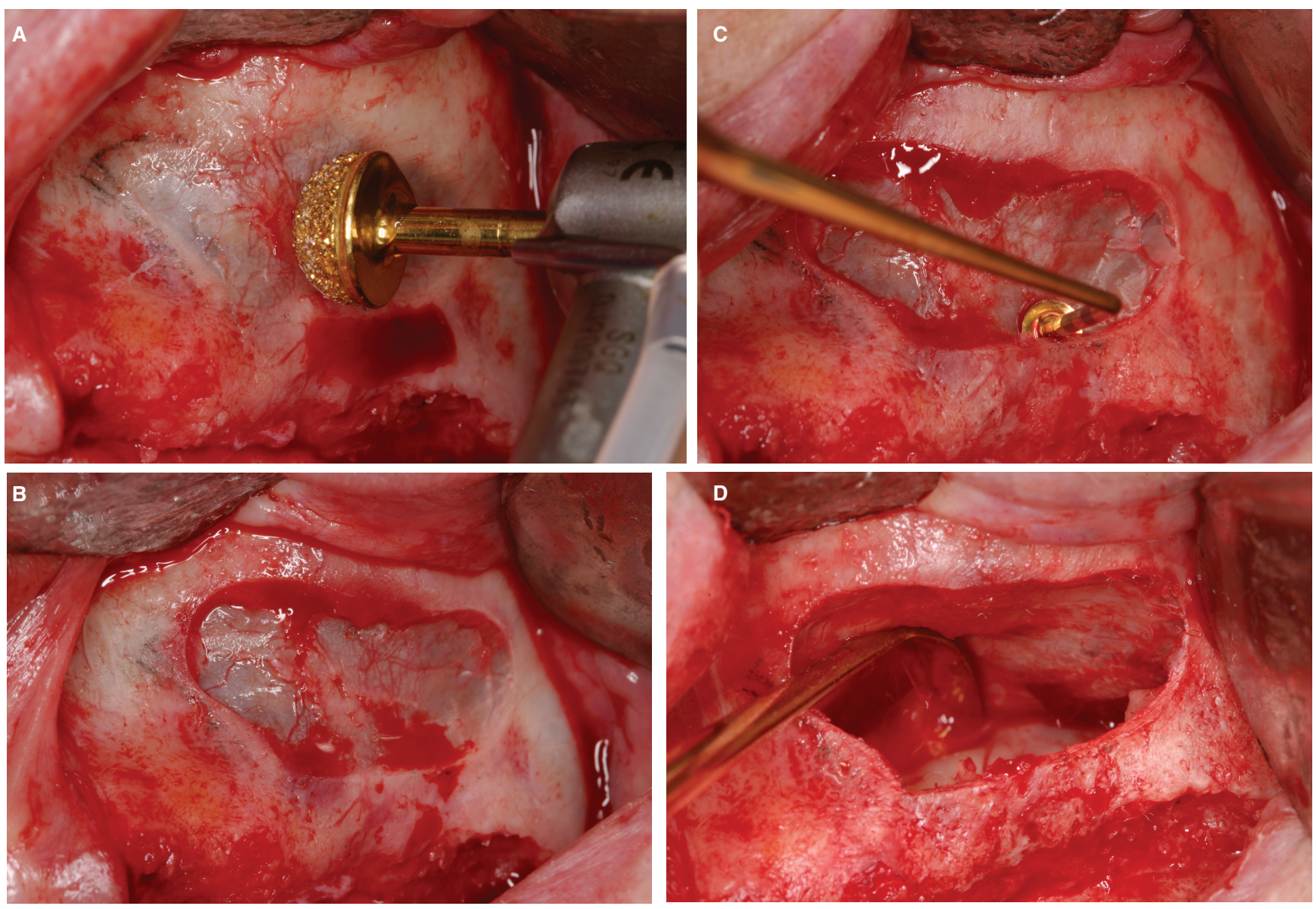

FIGURE 18 (A) Lateral movement of 8 mm DASK drill planes the bony wall to the desired shape. (B) Removal of all but paper-thin layer of bone. Microvasculature of lateral wall/membrane remain intact. (C) Dome-shaped elevator/separator in place. (D) Elevation completed up lateral wall. Source: Wallace et $\mathrm{al}^{11}$ Reproduced with permission from Elsevier

sutures to close the perforation, or growth factor-enriched biologic barrier membranes, such as leukocyte and platelet-rich fibrin (if blood is drawn at the time of surgery).

Many techniques have been reported for repair of perforated or torn Schneiderian membranes. ${ }^{77-82}$ These techniques are specifically chosen based on both the size and location of the perforation, and the perceived need to stabilize the repair to keep it securely in place. Without stabilization, it is possible for the repair to shift in position, or even be drawn up through the perforation into the body of the sinus during or after placement of graft material. The choice of a specific repair material will be based on the above factors, as well as on the physical characteristics of the material. Zijderveld et $\mathrm{al}^{62}$ and Shlomi et $\mathrm{al}^{80}$ preferred to use lamellar bone sheets for repairs owing to the material's rigidity. The following discussion is based on the paper by Testori et al, ${ }^{81}$ which presents 4 specialized repair techniques for larger perforations using bioabsorbable collagen barrier membranes.

The following generalizations should be helpful when attempting repairs:

- very small perforations may self-repair by membrane foldover or clot formation.
- large perforations will require large repairs for stability.

- large repairs tend to tent superiorly when grafts are placed.

- repair membranes placed near the lateral wall tend to shift medially when graft is placed.

- repair membranes that are soft and shapeless when wet are not ideal for large repairs.

It is not uncommon to perforate the Schneiderian membrane with high-speed rotary instruments (diamond burs) when performing a lateral window osteotomy. With careful membrane elevation, it is possible that these perforations will remain small. When the membrane elevation is complete, the small perforation will either disappear in the folds of the elevated membrane or, more likely, self-repair with a small blood clot. In this type of case a separate repair procedure is not indicated as the goal of graft material containment has been biologically achieved. If a very small perforation is still evident, it can be repaired using a biologic leukocyte and platelet-rich fibrin matrix (Intra-Lock International Inc., Boca Raton, FL, USA), or covered with a soft repair membrane such as CollaTape ${ }^{\circledR}$ (Sulzer Dental, Plainsboro, NJ, USA) or GelFilm ${ }^{\circledR}$ (Upjohn Company, Kalamazoo, MI, USA). 

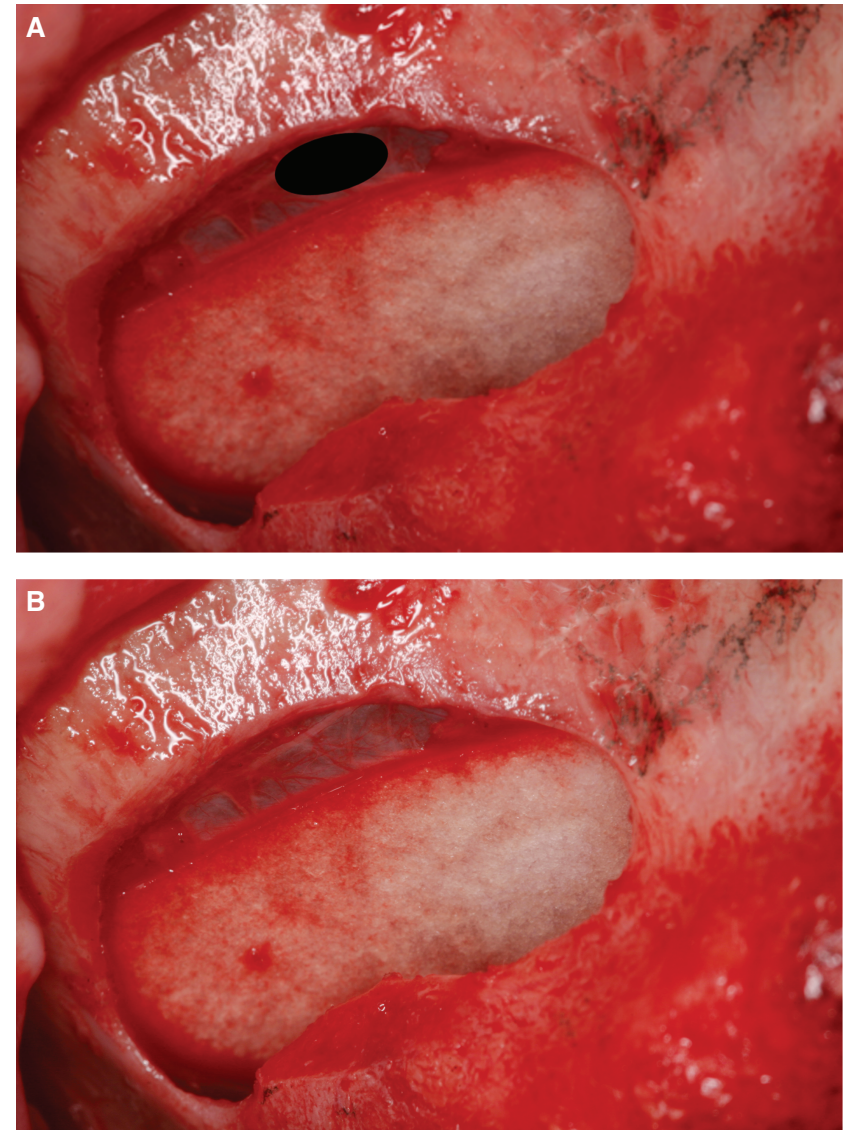

FIG URE 19 (A) Small-sized perforation. (B) Nonstabilized collagen repair forming a new roof confining the particulate graft

If the perforation is large (> $5 \mathrm{~mm}$ ), one should use a bioabsorbable membrane that retains its shape (BioGide ${ }^{\circledR}$; Geistlich Pharma NA, Inc. Princeton, NJ, USA) or remains stiff when wet, such as BioMend $^{\circledR}$ (Zimmer Dental, Carlsbad, CA, USA), OsseoGuard ${ }^{\circledR}$ (BioMet 3i, Palm Beach Gardens, FL, USA), Dentium ${ }^{\circledR}$ Collagen Membrane (Dentium USA, Cypress, CA, USA), or similar absorbable membranes. The amount of stability that can be achieved with the repair is directly proportional to the amount of coverage over the intact portion of the sinus membrane. There is no reason to avoid making a new roof for the graft material compartment with the repair membrane because it has been shown in animal studies that the elevated Schneiderian membrane plays a minimal role in vascularization and bone formation in the graft. ${ }^{83,84}$ In Figure 19A,B, an example is shown in which a collagen repair membrane forms a new roof in the damaged graft material compartment. Note that the Schneiderian membrane has been elevated to the horizontal position, demonstrating release of Schneiderian membrane from the medial wall.

As perforations become still larger (rents or tears of $>10 \mathrm{~mm}$ ), nonstabilized repairs become unpredictable, as they tend to shift medially while packing the graft material and may even rise upwards, through the tear, with partial or complete loss of the graft material into the sinus cavity. This untoward event may lead to blockage of the ostium, postoperative sinusitis, or a sinus infection. Major loss of graft material containment may necessitate surgical re-entry for removal of all particulate graft material.

Repair techniques have been developed to address both larger tears and tears in difficult locations. If, after final membrane elevation, the perforation resides close to the lateral, superior aspect of the window preparation (Figure 19A,B), it is quite common for the repair membrane to shift medially while the particulate graft material is being placed. This is caused by the convex shape of the lateral wall in the malar eminence (first molar) area, the upward tenting of the membrane when packing, and the likelihood that the repair membrane is not sufficiently wide to reach the medial wall. To counteract this tendency of the repair membrane to shift, a large membrane (usually an adjusted $20 \times 30 \mathrm{~mm}$ size) should be used, with a portion of it left outside the window and folded in a superior direction, and with it resting on the medial wall (Figure 20A,B). This is a simple repair modification that will prevent any medial and/ or superior shifting of the membrane with concomitant loss of graft material into the sinus proper.

In some instances, further stabilization can be achieved by a combination of the above-described folding technique with external tacking and/or internal suturing. Again, the membrane elevation must be completed to expose the bony walls and their vascular supply before completion of the repair. It must also be recognized that torn Schneiderian membrane is very fragile and therefore all suturing must be accomplished using small needles with minimal tension on the remaining membrane. Most often, it is not possible to suture the tear completely closed. When this is the case, it is possible to use the sutures as struts upon which to rest the repair membrane. The sutures can course between 2 sections of torn membrane or between the membrane and small holes drilled in the lateral wall (Figure 21A,B). Evidence of radiographic success of the repair procedure after 9 months is shown in Figure 21C,D.

In extreme situations, there may be insufficient residual membrane fragments to retain a suture. At this point, a decision has to be made as to whether to abort the procedure or perform a more extensive repair. In the following case (Figure 22A-D) the Loma Linda pouch technique ${ }^{79}$ was used, along with additional stabilization tacks ${ }^{81}$ to create a complete container for placement of the graft material. A large, $30 \times 40 \mathrm{~mm}$, BioGide ${ }^{\circledR}$ membrane (Geistlich, Wolhusen, Switzerland) was pushed through the window to create an internal sinus pouch to hold and confine the graft material. The edges of the membrane were left outside the window to hold it in position. Two tacks were also required to keep the entire membrane from slipping into the sinus and through the perforation.

The choice of a particular repair membrane will often be made by operator preference. General guidelines as to the type and location of the defect will be helpful in making this choice. In most cases, a membrane that retains its stiffness and shape when wet is advisable. This membrane will stabilize by contact with the remaining, intact, Schneiderian membrane. With a Loma Linda type of repair, there is minimal or no remaining Schneiderian membrane. In this case, a soft, moldable membrane is desired to make intimate contact with the available bony walls and create the "pouch-like" space for the particulate graft material. 
There is a relatively large literature base pertaining to implant survival following perforation and repair of the sinus membrane. Papers by Proussaefs et al, ${ }^{76}$ Jensen et al, ${ }^{85}$ (report of the sinus consensus conference of 1996) and Khoury ${ }^{86}$ state that implant survival is negatively affected by membrane perforations. Hernández-Alfaro et $\mathrm{al}^{31}$ report that the implant survival rate is inversely proportional to the size of the membrane perforation. Other authors ${ }^{63,80,81}$ present data showing that survival rates are not affected by perforations. The latter has been the experience at the New York University Department of Periodontology and Implant Dentistry when proper repairs are made and they remain intact throughout the postsurgical healing period and do not affect the implant survival rate. A study by Froum et al $^{89}$ reported that the average percentage of vital bone was $26.3 \pm 6.3 \%$ in perforated (repaired) sinuses vs $19.1 \pm 6.3 \%$ in nonperforated sinuses. While this difference was significant, there was no significant difference in implant survival rates.

The presence of a bioabsorbable repair membrane against the elevated Schneiderian membrane does not impede the blood supply to the graft as the reflected Schneiderian membrane does little to provide a blood supply. The Loma Linda pouch technique, however, presents a theoretical problem in that the repair membrane completely surrounds the graft and is likely at least to delay the vascularization of the graft from the lateral sinus walls. The vital bone formation in
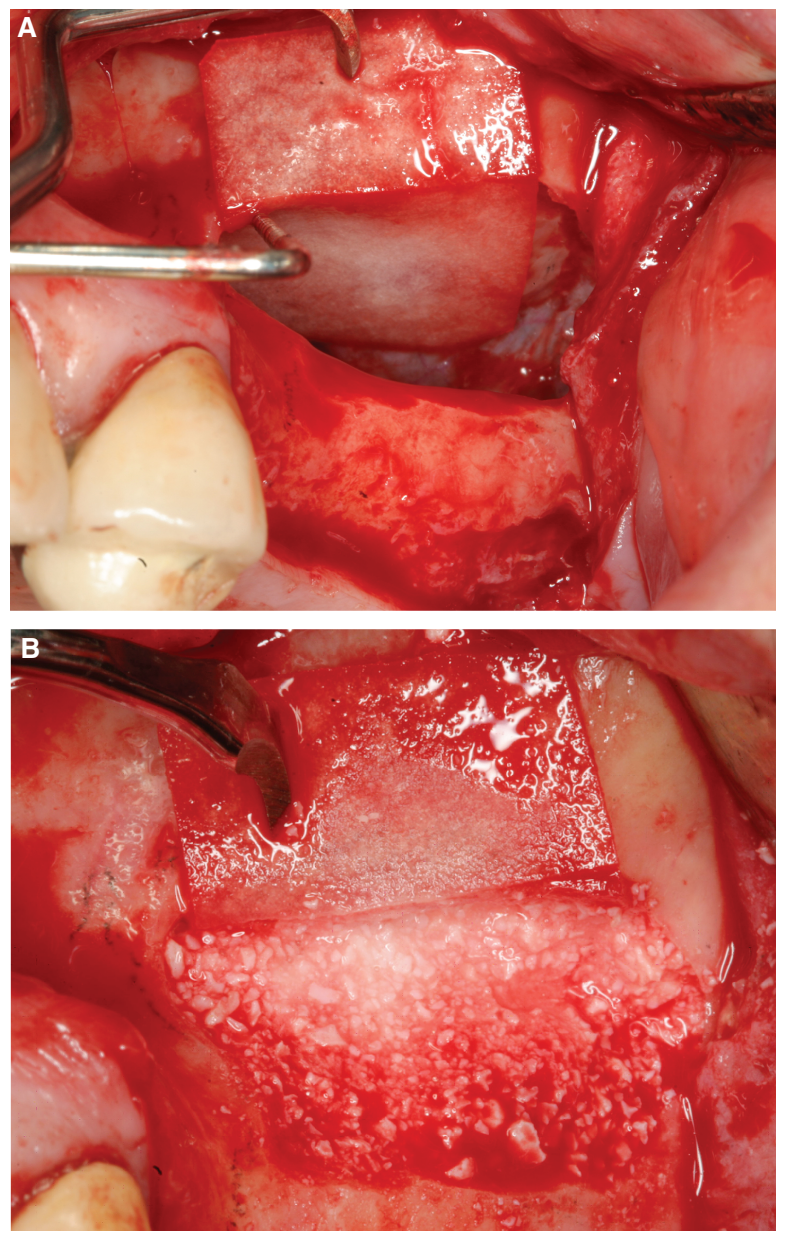

FIGURE 20 (A) Portion of repair membrane remains outside sinus to prevent medial shift. (B) Particulate graft in place the 2 large repairs presented above was $30 \%$ and $32 \%$ by volume, respectively, which is considered a favorable result when using $100 \%$ xenograft. Testori et al ${ }^{81}$ presented the results from 20 patients after repair of large perforations. All patients had minimal postoperative symptoms, and all showed clinical, histologic, and radiographic evidence of successful sinus elevation with $100 \%$ implant survival.

If repair procedures do not appear to give a stable result, it may be necessary to abort the grafting procedure and allow the sinus membrane to heal. A reasonable waiting time, confirmed by ear, nose, and throat physicians, is around 4 months (or 2 months for smaller perforations). Should this be the treatment of choice, the placement of a bioabsorbable barrier membrane over the window may prevent softtissue encleftation into the sinus cavity. It will probably be necessary to perform a split thickness re-entry flap over the window owing to the likelihood that the periosteum may be joined to the newly formed Schneiderian membrane in the window area. The residual small amount of soft tissue is then elevated, along with the Schneiderian membrane, to create the roof of the graft material compartment. This can be covered with a bioabsorbable collagen barrier membrane to isolate this small amount of connective tissue from the graft (Figure 23).

Another repair technique involves the utilization of autogenous leukocyte and platelet-rich fibrin membranes fabricated using the IntraSpin ${ }^{\circledR}$ System and protocol (Intra-Lock). The patient's blood is drawn, spun in a calibrated centrifuge, and a fibrin clot is obtained by compression. The compressed fibrin clot is resilient and pliable, and can be cut or pieced together to make a biologically active repair membrane that is rich in platelets, leukocytes, growth factors, and cytokines. ${ }^{90-92}$ The prepared membranes have a strong adhesiveness, allowing them to join together to facilitate the repair of large perforations. The fibrin membranes have sufficient strength to make it convenient to join them together by suturing (Figure 24A-D).

\subsection{4 | Mucous retention cysts}

Mucous retention cysts are a fairly common occurrence in the maxillary sinus. In a tomographic study by Maestre-Ferrin et $\mathrm{al}^{93} \mathrm{ra}^{9}$ diographic abnormalities were observed in $38 \%$ of observed cases, with $10 \%$ being mucous retention cysts. These cysts are not, by their presence alone, a complication or contraindication for maxillary sinus elevation. They may become problematic when they are elevated during sinus grafting and, as a result, block sinus drainage through the ostium. The likelihood of this outcome can be detected by performing a preoperative computed tomography analysis. The presence of cysts is readily detected and they can be diagnosed as not being a potential problem (small volume), a complication that can be handled at the time of surgery by drainage with a large-gauge needle, or a problem that must be treated via functional endoscopic sinus surgery prior to sinus elevation surgery. These lesions are unlikely to respond to antibiotic or anti-inflammatory medications and it is therefore prudent to refer the patient to an ear, nose, and throat specialist to diagnose and/or treat these conditions prior to sinus augmentation surgery. $A$ healthy sinus with a thin membrane and patent ostium is shown in Figure 25. Figure 26A,B radiographically 

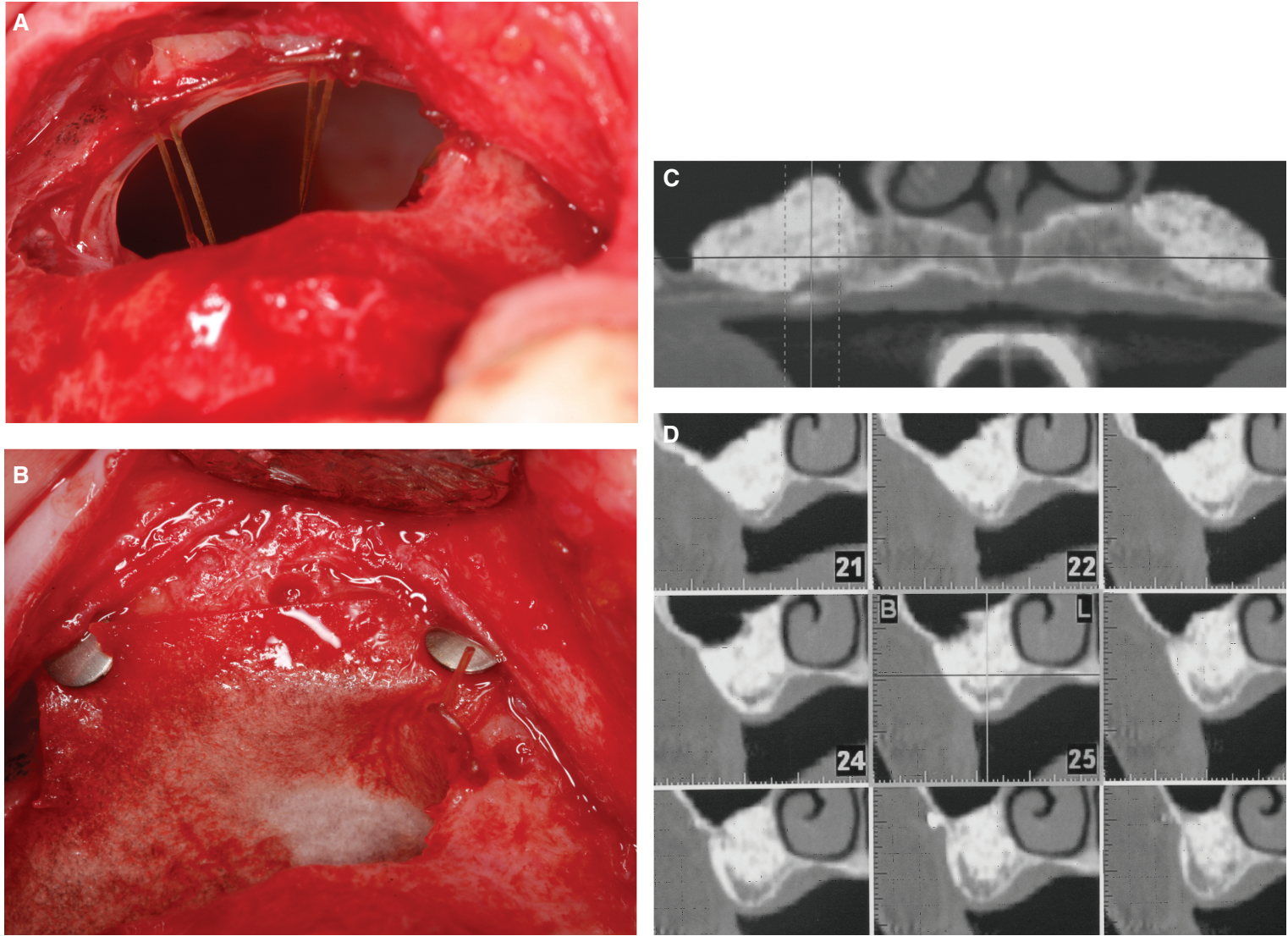

FIG URE 21 (A) Suture repair from a torn sinus membrane to holes made in the lateral wall. (B) Membrane resting on suture struts with additional fold and tack stabilization. (C) Nine-month postoperative panoramic computed tomography view (right side). (D) Nine-month postoperative cross-sectional computed tomography view. Source: Testori et al ${ }^{34}$ Reproduced with permission from Quintessence

demonstrates a mucous retention cyst and polyps. Mucous retention cysts and polyps can be differentiated from each other by form and location. Cysts are typically dome-shaped and arise from the sinus floor. Polyps typically have a pedunculated base and arise from the sinus walls. A yellow fluid aspirant is pathognomonic for a sinus cyst.

A generalization can be made that a cyst which occupies twothirds of the total sinus volume is likely to block drainage through the ostium if elevated. If it is determined that elevation of the cyst will lead to a complication (postoperative sinusitis as a result of blockage of sinus drainage), there are 2 distinct treatment options: the first is functional endoscopic sinus surgery prior to sinus elevation to remove/marsupialize the cyst; and the second is to aspirate the contents of the cyst at the time of sinus elevation surgery. A lateral window is created by a complete osteotomy technique involving total removal of the window. Access is now present for insertion of a 22-gauge needle through the sinus membrane and into the cyst to remove the contents via aspiration (Figure $27 \mathrm{~A}-\mathrm{D}$ ).

A question remains as to whether intraoperative aspiration, which leaves the cyst lining in place, can be as effective as endoscopic marsupialization, which removes a majority or all of the cyst lining. A study by Hadar et al, ${ }^{94}$ which followed up on endoscopic cyst removal, showed re-formation of the cyst in $3 \%$ of patients. A study by Testori et $\mathrm{al}^{95}$ followed patients treated by intraoperative cyst aspiration for 1-3 years after maxillary sinus elevation. Only cysts that were $>1 \mathrm{~cm}$ along the long axis and located in the area to be elevated were included in the study. Fifteen patients were included, the mean follow-up was 8 years, and no intra- or postoperative complications occurred. Postoperative disappearance of antral cysts was radiologically documented in 12 patients whose Schneiderian mucosal thickness ranged from 1 to $2 \mathrm{~mm}$ (according to analysis of computed tomography scans) after a 6-month healing period (Figure $28 \mathrm{~A}, \mathrm{~B}$ ). In the remaining 3 patients, a computed tomography scan performed 6 months postoperatively showed the presence of residual antral cysts with a reduced size that did not affect implant survival rates. There was no occurrence of sinusitis after deflation, and no new complications occurred intra- or postoperatively. Thirty-one implants (5 in a 1-stage procedure and 26 in a 2-stage procedure) were placed 6 months after the sinus surgery; only 1 failure occurred (mobility, 1 month after insertion). In January 2014, the cumulative implant survival rate was $96.8 \%$.

\subsection{5 | Other intraoperative complications}

Complications, such as tears in the buccal flap and injury to the infraorbital nerve, generally result from poor surgical technique. Buccal flap tears may result from attempts to release the flap to achieve primary closure. This is usually unnecessary in a typical sinus elevation 

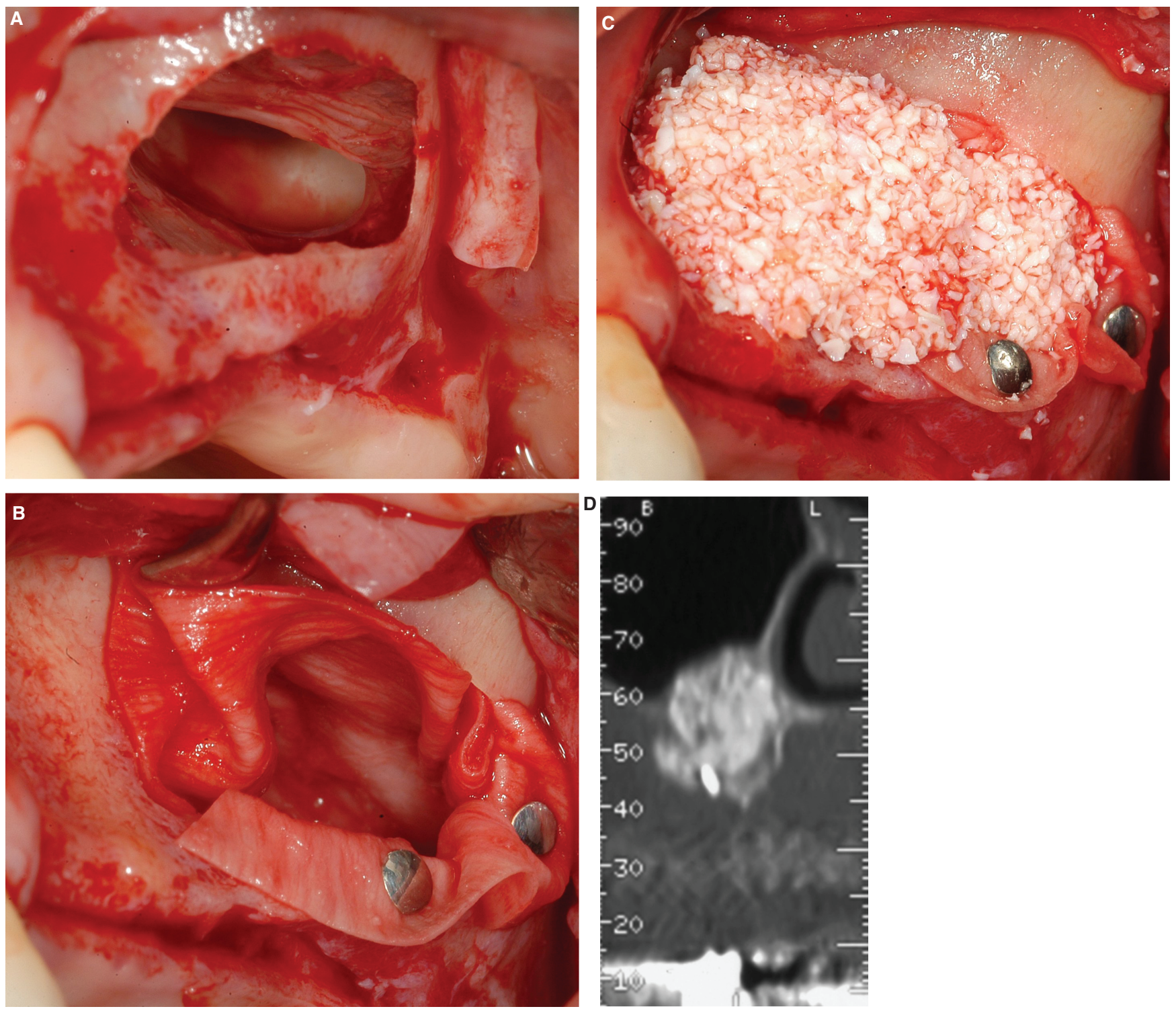

FIG URE 22 (A) Large membrane tear. (B) $30 \times 40$ mm BioGide membrane in position. (C) Particulate Bio-Oss graft in place. (D) Six-month postoperative cross-sectional computed tomography image. Source: Testori et al ${ }^{34}$ Reproduced with permission from Quintessence

procedure. As there is no change in external dimensions, the flap will close tension free without release. Loss of primary closure is more often a problem when simultaneous ridge augmentation is performed. $\mathrm{Be}$ aware of the possibility that the flap may be thin in the area of release and that the direction of the bone surface changes in the area of the malar eminence. Blunt or pressure injury to the infraorbital nerve may occur during flap retraction. If the flap elevation extends superiorly to this position, the exit of the nerve from the bone should be visualized and retraction placed distal to it. It is also possible to injure this nerve during sharp dissection performed to release the flap for primary closure. The exit-point of the nerve from the skull is just below the infraorbital notch. Identifying the location of this anatomic structure is crucial before performing these procedures (Figure 29).

In cases of severe maxillary atrophy it is possible to find the nasal floor in a crestal location, where one would expect to find the maxillary sinus. The preoperative computed tomography scan shows that there is no residual crestal bone and that the proposed restoration in cross-section \#97 will not be located below the sinus, but beneath the nasal passage (Figure 30A). The postoperative axial view shows that, in addition to a posterior sinus graft, the nasal floor has also been grafted (Figure 30B). In this particular case, no remedial therapy was advised as the ostium remained patent and the nasolacrimal duct was undisturbed. This sinus was grafted with Puros ${ }^{\circledR}$ allograft. The entire graft (nose and sinus) resorbed, and only the sinus was regrafted 14 months later.

Best clinical practice includes:

- performing presurgical diagnosis with computed tomography scans to disclose difficult anatomy, vessel location, sinus pathology, and presence of cysts.

- making the window in the best location $(3 \mathrm{~mm}$ from the floor and anterior wall). 


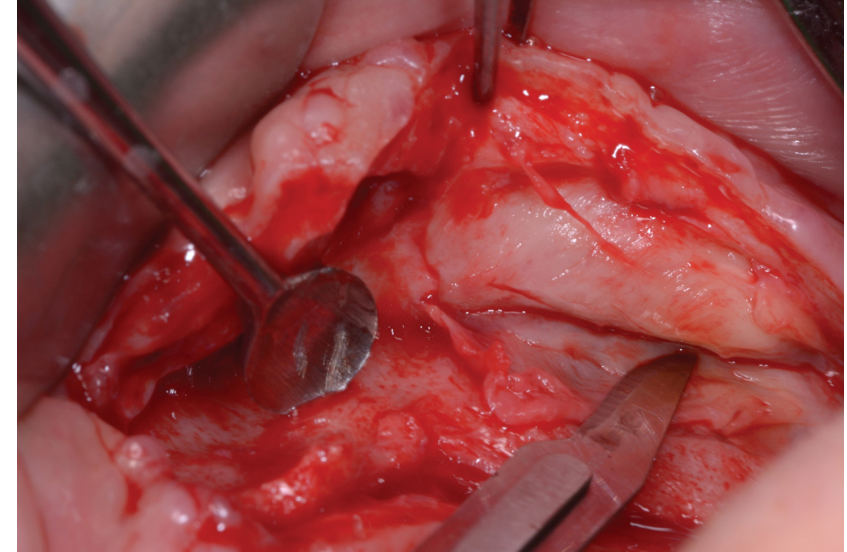

FIGURE 23 Split thickness flap over previous window location. Membrane now can be elevated along with small amount of periosteum

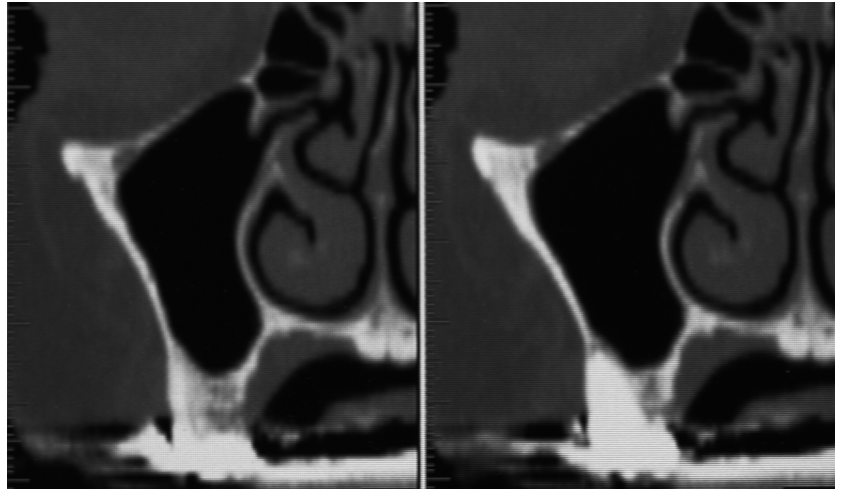

FIGURE 25 Healthy sinus with a patent ostium high on the medial wall

drainage.
FIGURE 24 (A), Leukocyte and platelet-rich fibrin as it is removed from centrifuge, prior to removal of red blood cells. (B) Compressed to form a leukocyte and platelet-rich fibrin membrane. (C) Multiple sinus membrane perforations. (D) Perforation biologically sealed. Photographs provided by R. Miller. Reproduced with permission from R. Miller
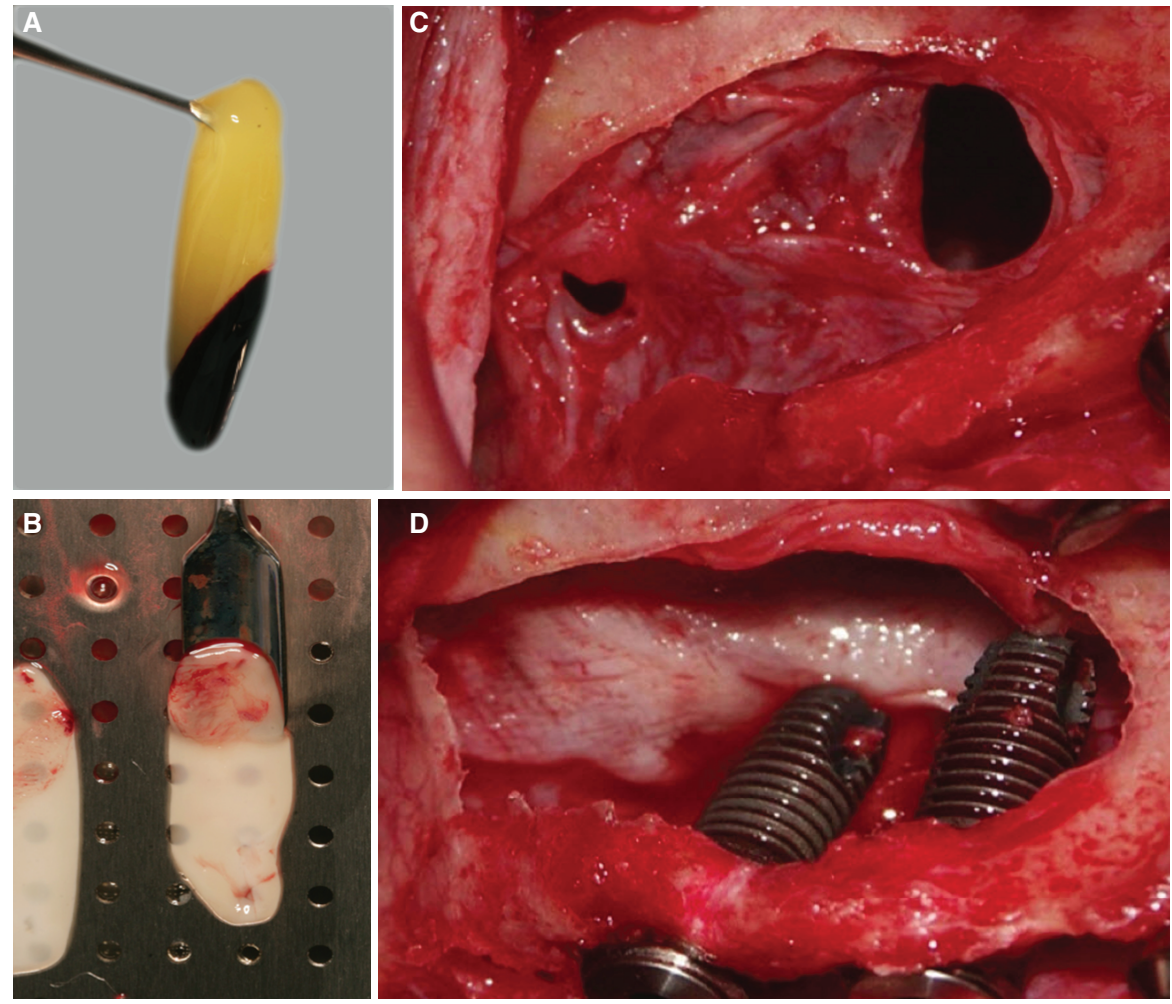

- using piezoelectric surgery or DASK ${ }^{\circledR}$ for lateral osteotomy and initial membrane elevation.

- elevating the membrane from lateral to medial, keeping the elevators on bone at all times.

- repairing perforations with collagen barrier membranes or biologic leukocyte and platelet-rich fibrin membranes, as appropriate.

- using a collagen repair membrane that remains rigid when wet to achieve the most stable repair. Leukocyte and platelet-rich fibrin is both resilient and adhesive.

- ensuring that all repairs are stable.

- aspirating mucous retention cysts if elevation might block sinus

\subsection{6 | Postoperative complications}

Postoperative edema, ecchymosis, mild-to-moderate discomfort, minor nosebleed, minor bleeding at the incision line, and mild congestion are within the scope of expected patient responses to this procedure. Some are caused by manipulation of the facial flap and others by manipulation of the sinus membrane.

Major postoperative complications after sinus elevation surgery are relatively uncommon. They include graft infections, sinus infections, postoperative sinusitis, profuse postoperative bleeding, flap dehiscence, oroantral fistula formation, formation of inadequate graft volume for implant placement, loss of graft material 

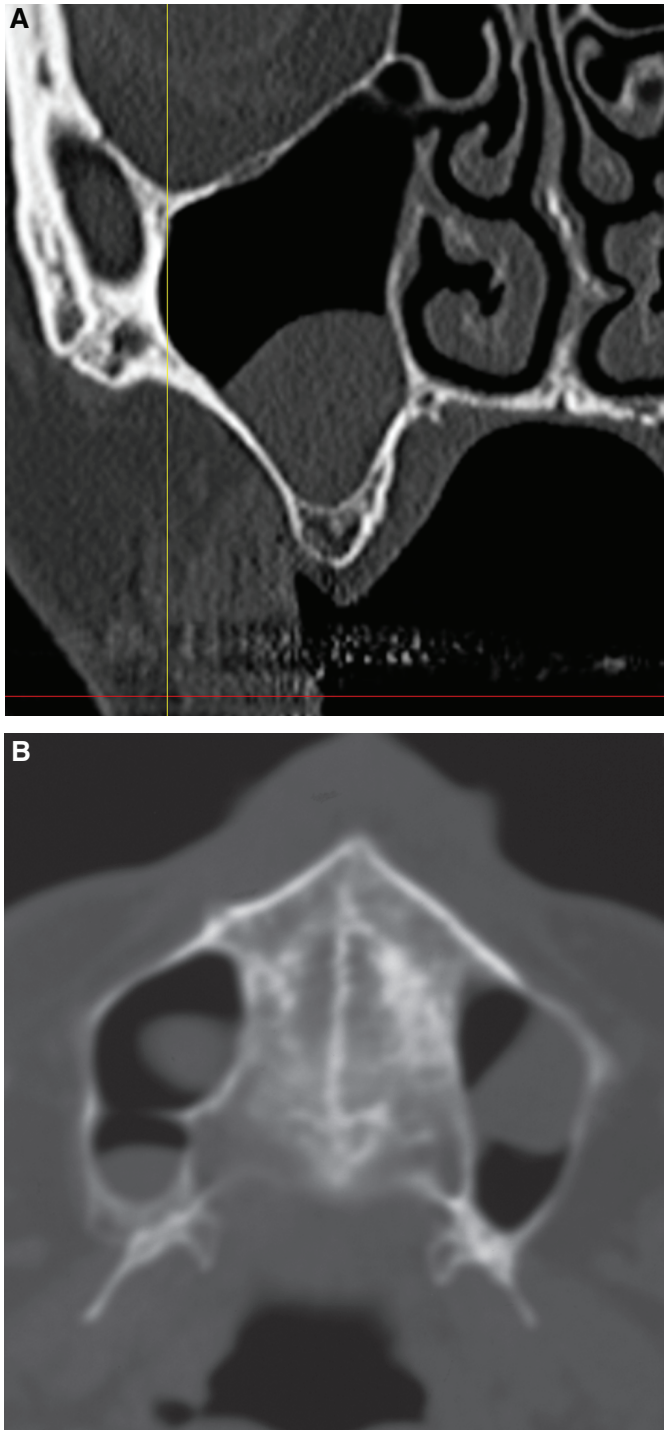

FIG URE 26 (A) Dome-shaped mucous retention cyst, cross sectional view. (B) Polyps originating on medial and lateral sinus walls, axial views

containment as a result of either sinus membrane rupture or exfoliation of graft material through the sinus window, maxillary cyst formation, ${ }^{96,97}$ migration of dental implants into the sinus graft, the sinus cavity proper, or the paranasal sinuses, ${ }^{98}$ and failure of dental implants.

A consensus conference by the Academy of Osseointegration ${ }^{99}$ concluded that maxillary sinus elevation was the most predictable of the pre-prosthetic augmentation procedures. They further concluded that complications were relatively few, generally localized, and easily remedied. While this is for the most part true, it should be appreciated that improper handling of complications may lead to more serious adverse outcomes, such as an intraorbital abscess or an intracranial abscess, or migration of implants into the maxillary sinus or paranasal sinuses ${ }^{98}$

In a prospective study of 100 consecutive sinus elevations, Zijderveld et $\mathrm{al}^{62}$ reported an $11 \%$ incidence of membrane perforations and a $2 \%$ incidence of bleeding as intraoperative complications when utilizing a rotary window technique. The postoperative complications listed in order of most frequent occurrence were loss of implants (4\%), wound dehiscence (3\%), graft infections (2\%), postoperative maxillary sinusitis (1\%), and loss of or inadequate graft volume (1\%). While postoperative complications are relatively infrequent, understanding how to cope with them may be vital for the ultimate success of the elevation procedure.

\section{6 | POSTOPERATIVE INFECTIONS}

\section{1 | Etiology and incidence of sinusitis / sinus infections}

Postoperative infections are relatively infrequent, with infection rates between $2 \%$ and $5.6 \%{ }^{62}$ reported, with often no distinction being made between a true sinus infection or a sinus graft infection. The incidence of postoperative infections can be reduced by utilizing protocols involving proper case selection (preoperative diagnosis), pre- and postoperative infection control with appropriate antibiotics, and sound surgical techniques. When infections are suspected, therapy should be rendered quickly and effectively in order to avoid serious adverse outcomes.

Infections after sinus elevation surgery can occur in 2 locations: the sinus and the sinus graft. Most commonly, the infection is not a true sinus infection but an infected sinus graft. It should be remembered that the sinus graft is not actually in the sinus, but is located below the elevated sinus membrane, hence the term subantral augmentation. True sinus infections are less common but may have more widespread consequences if not appropriately managed owing to the interconnectivity of the paranasal sinus network.

Postoperative sinus infections may commonly arise from 2 general sources. The first is through exacerbation of a previously existing, asymptomatic, chronic sinus condition (infection or inflammation) by the anticipated postoperative inflammatory changes. The second is from contamination, through a membrane perforation or tear, with bacteria from the oral cavity or infected sinus grafting material.

Preexisting inflammatory sinus disease (seasonal, tooth-related) may, under less than ideal conditions, be a factor in the etiology of postoperative sinus infection. A healthy sinus is considered to have a high "compliance," which may be defined as the ability of the sinus to respond and recover from a bacterial or inflammatory threat. An unhealthy sinus does not have the same level of innate ability to respond.

Common conditions that lead to acute sinusitis are blockage of the osteomeatal complex by inflammatory changes resulting from tooth-related problems (endodontic or periodontal) and seasonal allergic reactions. The most common causes of sinusitis following sinus elevation procedures are likely to be infection/inflammation relating to inadequately contained sinus grafts that have contaminated the sinus proper through recognized or unrecognized perforations, or 

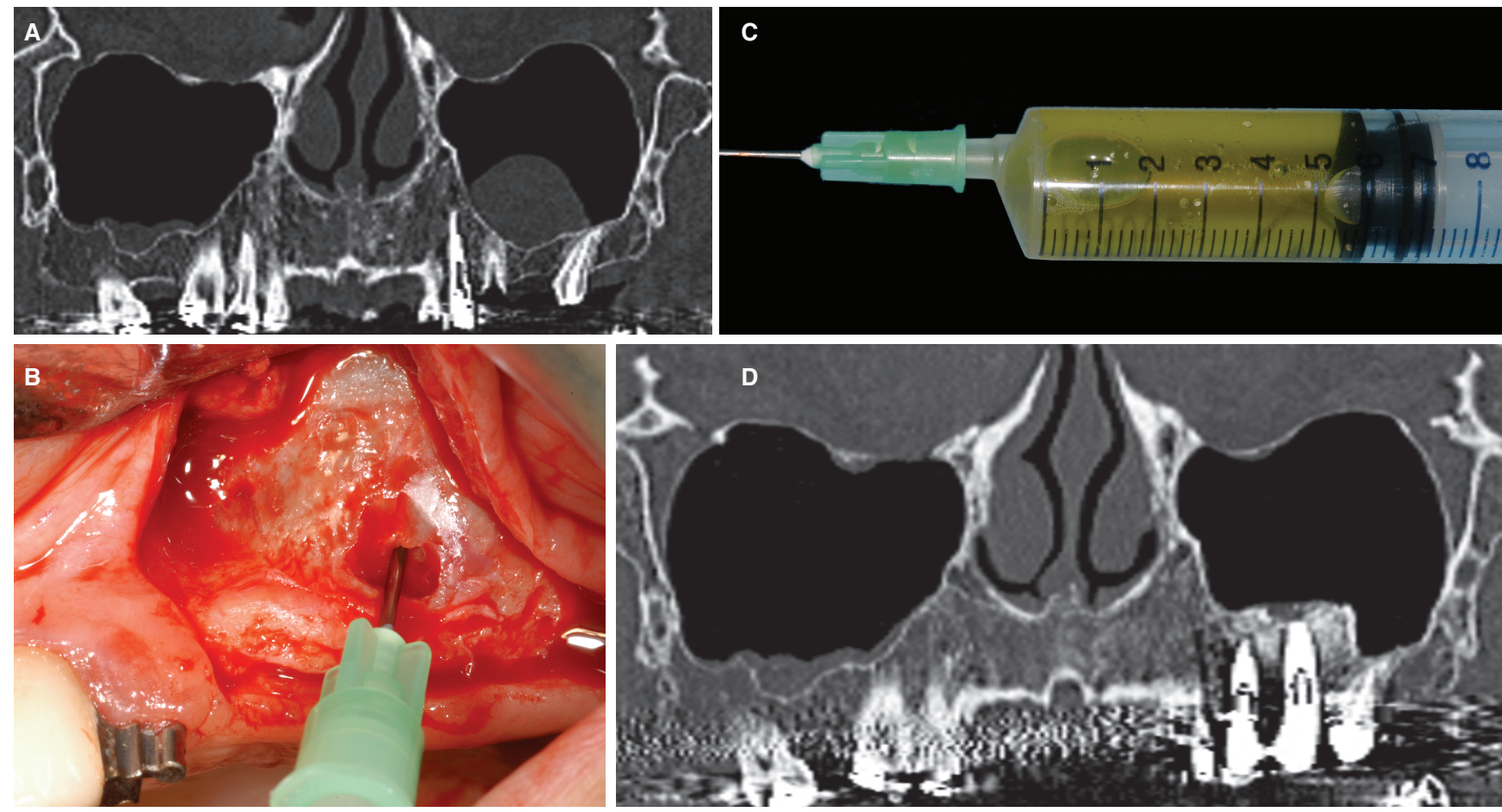

D
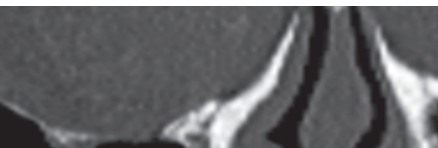

FIG URE 27 (A) Panoramic view again showing dome-shaped appearance of cyst on sinus floor. (B) 22-Gauge needle inserted through sinus membrane into cyst. (C) Typical yellow aspirate. (D) 2-y postoperative cross section. (reproduced with permission from Wiley-Blackwell)

FIGURE 28 (A) Preoperative cross sections of sinus with dome-shaped cyst present. (B) Same sinus 1 y after simultaneous cyst aspiration, sinus elevation, and implant placement
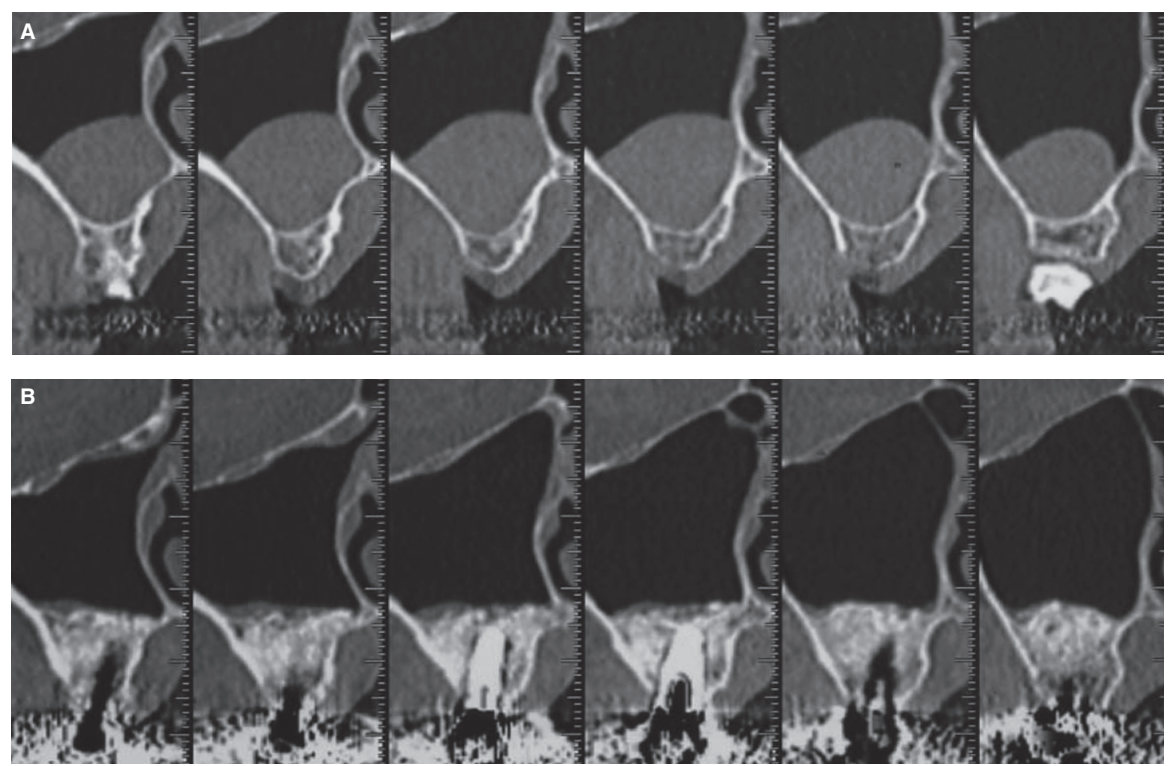

blockage of drainage by the elevation of mucous retention cysts or severely thickened sinus membranes.

In cases in which inflammation and/or infection from a recognized etiology (periodontal, periapical, allergic) are present, presurgical antibiotic/anti-inflammatory therapy, along with removal of the etiology, will in many cases resolve the problem before augmentation surgery. Figure $31 \mathrm{~A}-\mathrm{C}$ shows that the etiology of the sinus pathology was the infected molar teeth. These were removed and the patient was placed on a course of Augmentin and prednisone therapy, leading to almost complete resolution of the sinus pathology.

Patients with a preoperative diagnosis, or symptoms, of acute sinusitis or acute chronic sinusitis should receive or be referred for appropriate presurgical therapy; if referred, medical clearance should be obtained before sinus elevation surgery is performed. This therapy may be in the form of antibiotic therapy (Augmentin or Levaquin are appropriate), combined antibiotic and anti-inflammatory therapy (one such regimen might include Augmentin [875 mg 


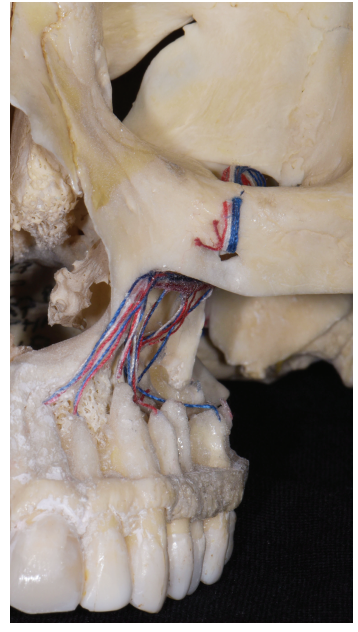

FIGURE 29 Location of the infraorbital foramen on a dry skull

of amoxicillin/125 mg of clavulanic acid, twice daily $\times 10$ days] and prednisone [ $40 \mathrm{mg}$ for 3 days, $20 \mathrm{mg}$ for 3 days, $10 \mathrm{mg}$ for 3 days]), endoscopic sinus surgery to remove pathologic tissues, or perhaps to widen the ostium to create more favorable drainage. It would not be considered inappropriate to take another computed tomography to determine if this therapy has been successful prior to performing the sinus elevation. Sinus elevation surgery will, in general, result in a short-term inflammatory reaction in the sinus, possibly compounding any previously existing pathology. This response is highly elevated when bone morphogenetic proteins are used as part of a sinus graft because cellular responses increase dramatically. The response is short-lived and will resolve without therapy (Figure 32A-C); however, it is advisable to inform the patient of this likely occurrence.

\subsection{1 | Etiology and incidence of sinus graft infections}

Sinus graft infection is the most common form of infection encountered after sinus elevation surgery. The incidence of postoperative sinus graft infections has not been separately documented but, by inference, it is approximately $2 \%-5 \%$. The most common symptoms may include local tenderness, nasal obstruction, pain, swelling, fistula formation, flap dehiscence, and suppuration from a fistula or the incision line. Increased intrasinus pressure may be a secondary factor, which might result in blocked sinus drainage. Symptoms may appear soon after therapy (within 2 weeks) or may first appear after a few months and sometimes, but not always, be proceeded by vague symptoms. Figure 33A,B presents a typical computed tomography scan appearance of a late (2-month) postoperative infection. A somewhat common appearance is that of a "black hole" in the central portion of the graft with a radiopaque dome or "halo" over the graft. Both views show what appears to be an undisturbed layer of graft material (normal dense opacity) surrounding the infected
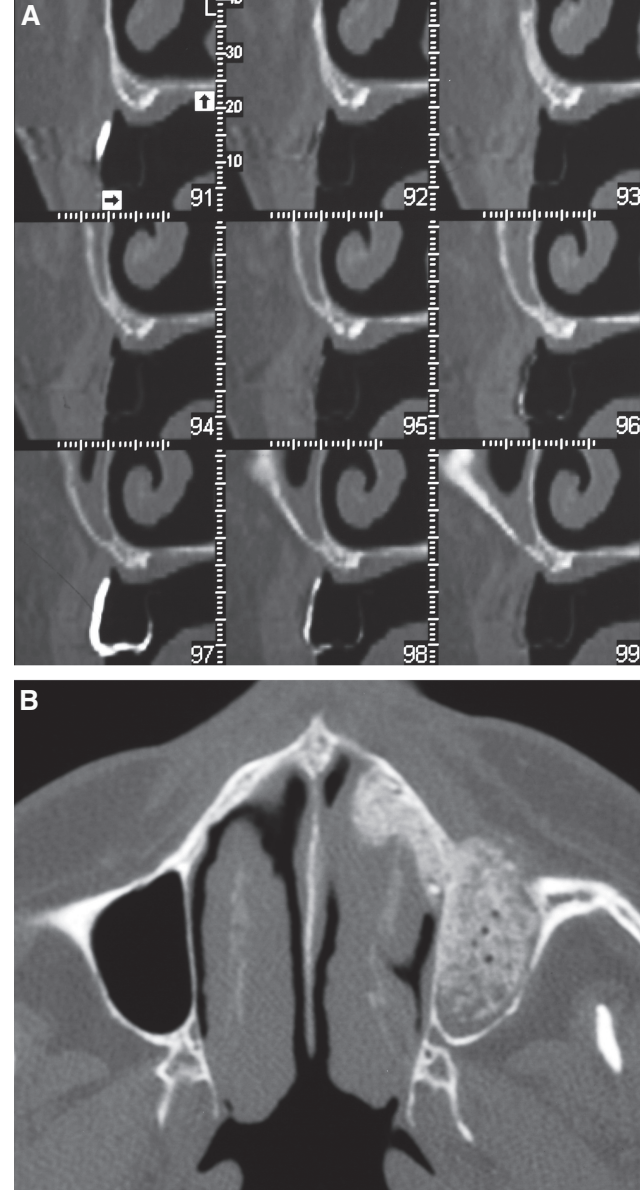

FIGURE 30 (A) Cross-sectional computed tomography-scan view indicating that the proposed restoration is not below the sinus. (B) Postoperative axial view showing the graft in both the sinus and the nasal passage

core. Upon open debridement, this dome feels quite solid and may not be easily removed.

Sinus graft infections may be caused by:

- preexisting sinus infection via perforation (should not treat symptomatic patient).

- contamination of the surgical site:

- salivary/bacterial contamination of the graft material, instruments, or membrane

○ untreated periodontal disease

o adjacent periapical pathology

- lapses in the chain of sterility

o extended surgical time.

- infected simultaneous lateral ridge augmentation procedures.

\subsection{2 | Prevention of sinus graft infections}

As an infected sinus graft can be a catastrophic event for a patient in terms of morbidity, additional therapy, increased treatment time, 
A

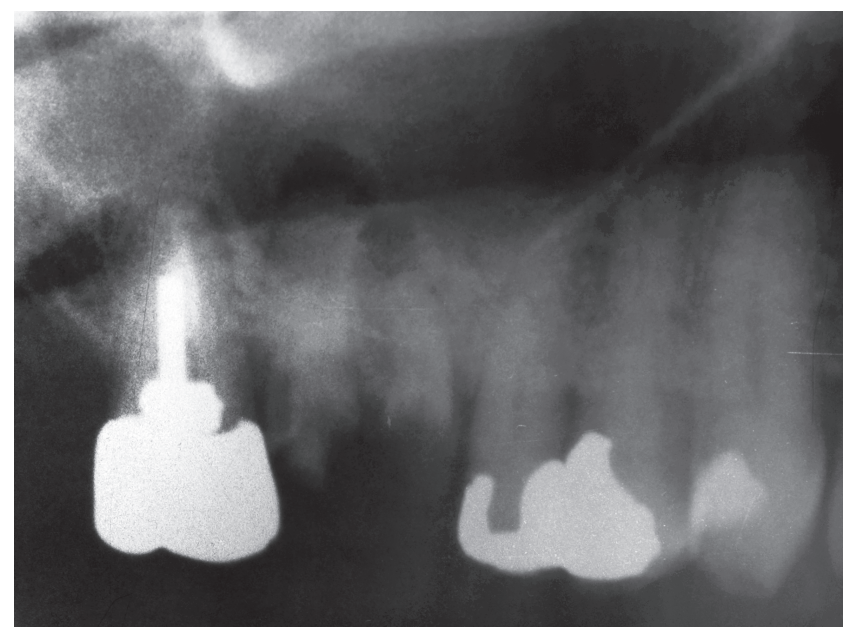

B

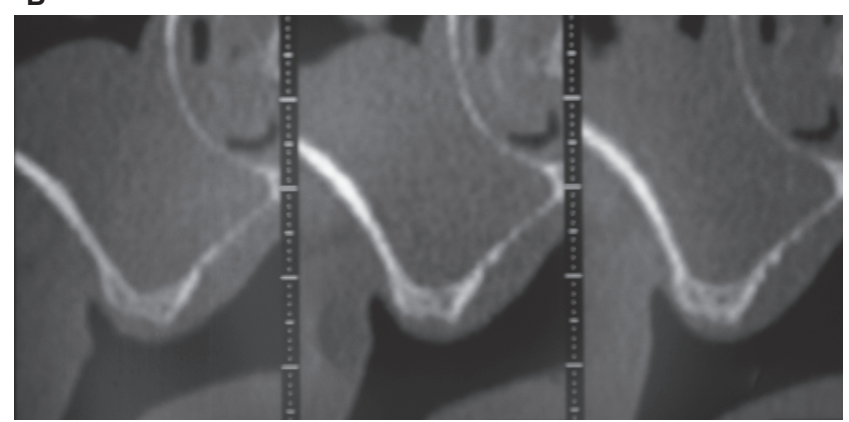

C

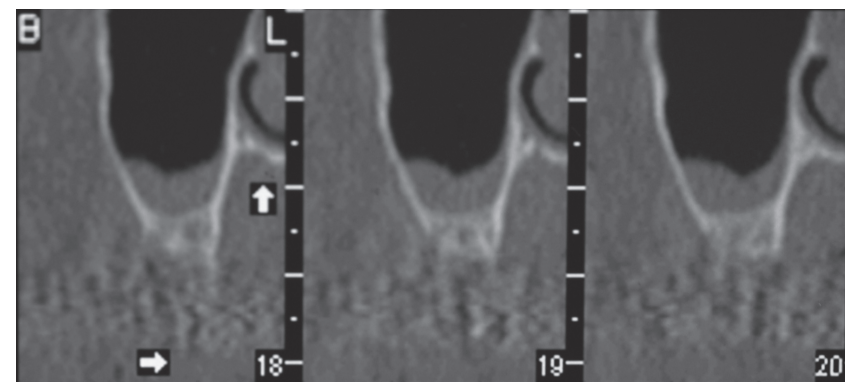

FIGURE 31 (A) Hopeless molars in a panoramic view. (B) Computed tomography scan after extraction of the molars. (C) Computed tomography scan 2 mo after Augmentin/prednisone therapy

and possible systemic complications, all efforts should be made to prevent this untoward outcome.

Preoperative diagnosis of potential sources of graft infection is invaluable. Preexisting periapical pathology, when the apices of infected teeth are in (or close) to the sinus, produces a reaction in the sinus that may be one of inflammation and/or bacterial contamination (Figure 34). When the membrane is elevated, these bacteria are immediately enclosed in a bone graft placed in a confined space; an ideal incubator. Localized endodontic and periodontal therapy should be completed before sinus grafting, or the hopeless teeth should be extracted.

Simultaneous extraction of teeth that penetrate the sinus floor may open a pathway to infection as the sinus graft is immediately
A
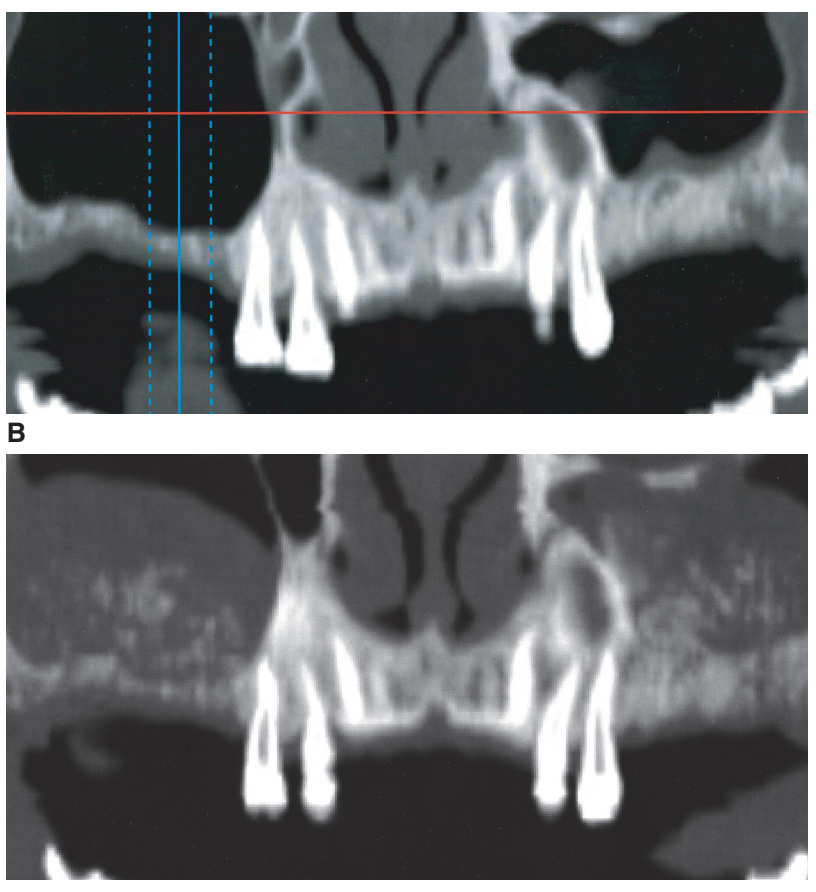

C

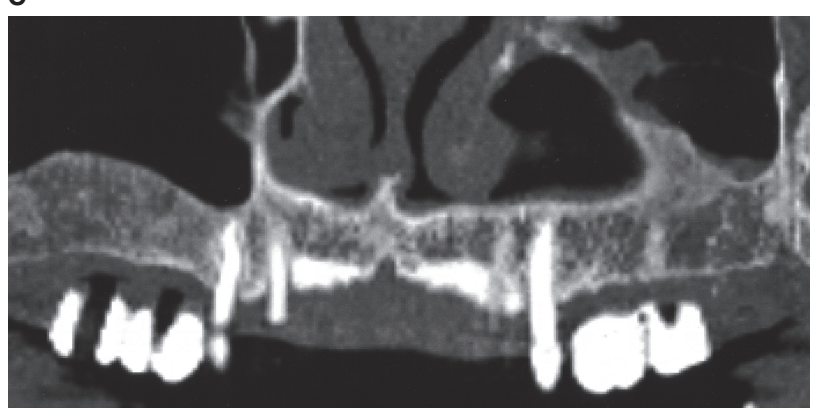

FIGURE 32 (A) Preoperative computed tomography. (B) 1 wk following sinus elevation with recombinant bone morphogenetic protein-2/acellular collagen sponge in combination with a mineralized bone replacement graft + allograft. (C) 4 mo following sinus lift

connected to the oral cavity through the extraction socket, which may or may not be covered by a flap release with primary closure. Sinus grafts with simultaneous ridge augmentation procedures are a further extension of the above extraction socket scenario. Barone et al $^{101}$ reported on 124 sinus elevations, 26 with simultaneous lateral ridge augmentations. The infection rate was $3 \%$ for the sinus graft only group ( $\mathrm{n}=98$ ) and $15.4 \%$ for the group that had simultaneous ridge augmentations ( $n=26)$. Five of the 7 infections occurred in smokers. The cause of the infection in these patients and in other ridge augmentation studies has been attributed to the breakdown of primary soft-tissue closure over the grafted site with exposure of the barrier membrane and subsequent graft contamination. It should be noted that in a ridge augmentation procedure, the incision line is directly over the barrier membrane, while in a properly designed sinus graft the membrane should be distant from all incision lines. Soft-tissue healing appears to be affected in a negative way by smoking, but smoking alone has not been shown to be a negative factor in pure 

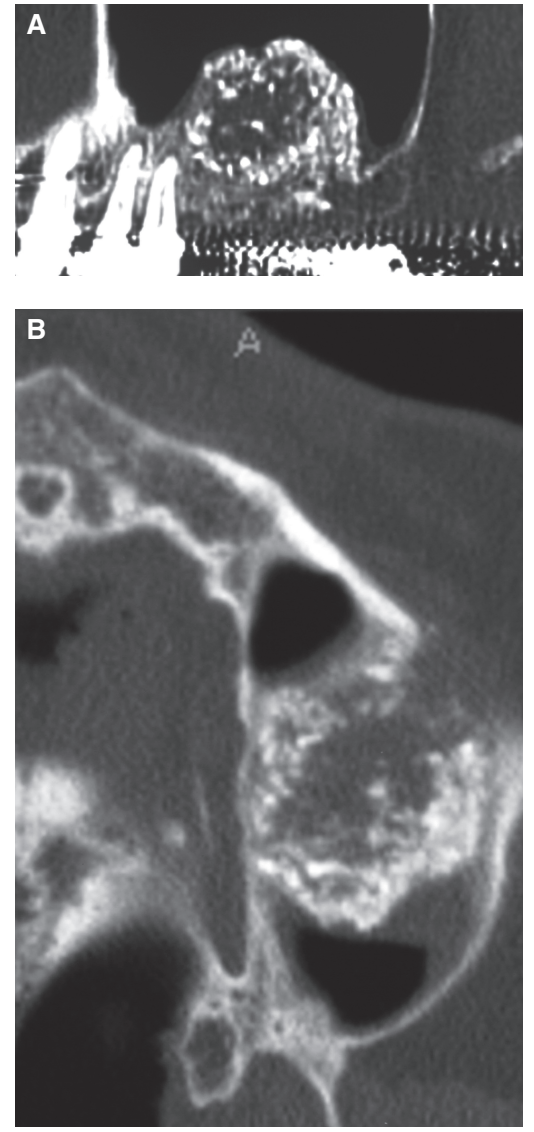

FIGURE 33 (A) Panoramic computed tomography view of an infected graft, depicting a "black hole". (B) Axial view depicting an infected central core surrounded by graft material

sinus grafting procedures. In a study by Levin et al, ${ }^{102}$ onlay bone grafts had a higher complication rate in smokers than in nonsmokers but there was no such relationship in pure sinus lift grafts.

Another overlooked source of graft contamination is the utilization of nonsterile instruments, either directly in the sinus or to manipulate the particulate graft material. Instruments that have been sterilized prior to the surgical procedure do not remain sterile once they have been introduced into the oral cavity. A common procedural error is to utilize an instrument, such as a periosteal elevator, that has been utilized in the mouth to hydrate/mix the graft material or carry it to the sinus. This is a breakdown in sterile surgical protocol that must be avoided.

Prophylactic procedures play an important role in prevention of infection. Many antibiotic regimens have been recommended for this purpose. In the author's experience, amoxicillin-clavulanate (Augmentin) is the drug of choice. The spectrum is greater than that of amoxicillin or ampicillin owing to the presence of clavulanic acid, which is active against $\beta$-lactamase-producing bacteria. Augmentin 875/125 mg twice daily for 7-10 days (starting the night before surgery) is an effective prophylactic dose. Historically, clindamycin (Cleosin) has been recommended for penicillin-allergic patients. However, some clinicians believe that clindamycin is not the ideal prophylactic antibiotic for these patients. In all our authors'
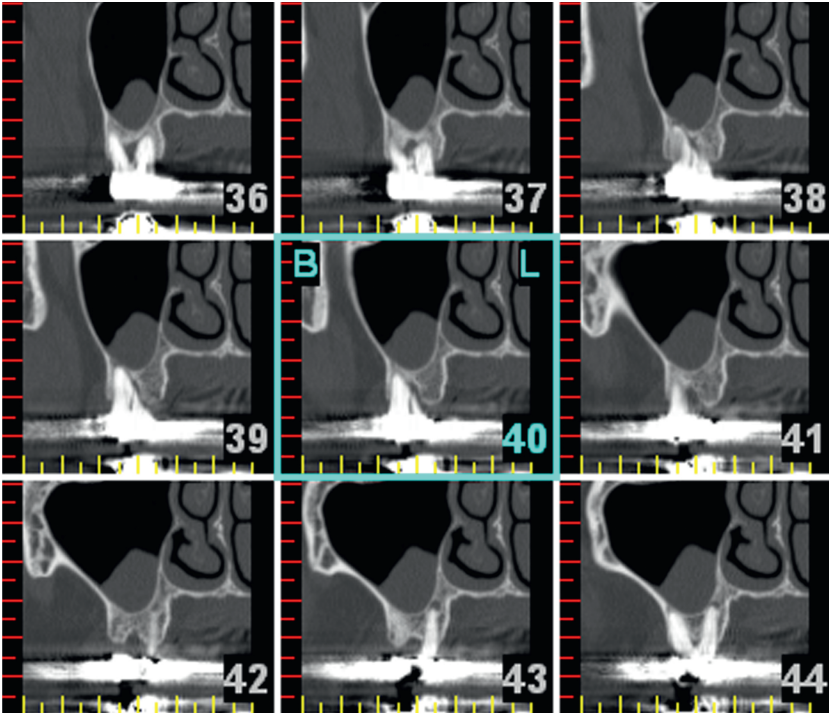

FIGURE 34 Periapical pathology extending into the sinus

experience over more than 28 years of sinus grafting, the majority of observed or reported infections occurred in patients taking prophylactic clindamycin or no antibiotic at all. The authors have used levofloxacin (Levaquin) or moxifloxacin (Avelox), second- and third-generation bactericidal fluoroquinalones, with much more favorable results. As there have been numerous reports of Achilles tendon rupture, tendonitis, and peripheral neuropathies following use of fluoroquinalones, especially when used in conjunction with steroids, ${ }^{103,104}$ the use of Zithromax or Biaxin, both bacteriostatic macrolides, may be an alternative for penicillin-allergic patients. In general, the sinus graft infection rate appears to be higher in penicillin-allergic patients. An unrelated study by Wagenberg \& Froum ${ }^{105}$ reported an infection rate 3.3 times higher after immediate implant placement when amoxicillin could not be used owing to a history of allergy. The following recommendations are given as measures to reduce the incidence of postoperative infection. (Table 6)

\subsection{3 | Treatment}

Treatment of sinus graft infections should begin immediately after symptoms are recognized. The most common symptom is swelling over the lateral window site. Other symptoms include localized pain and/or tenderness, fistula formation, flap dehiscence, and suppuration. Sinus graft infections usually occur within the first 2 weeks after therapy. Late infections (1-6 months) occur less frequently. In general, infections are quite evident with reported patient discomfort and observed clinical swelling. Sometimes the symptoms are less evident, with drainage occurring through a small fistula in the area of the lateral window. On other occasions the symptoms are so mild (nonspecific mild discomfort) that the diagnosis is delayed for up to 1 month or longer. Early treatment is essential as the partial or total loss of the graft is a possible negative outcome. Other negative outcomes include the occurrence of an oroantral fistula, which will require surgical correction, and 
the possible development of a sinusitis as a result of loss of graft containment.

Treatment can generally be described as involving 4 stages, each more invasive than the other, which are performed sequentially, as needed, until the infection resolves. The waiting time between stages is in the order of 7-10 days at a maximum, as positive effects should be noted by that time. The 4 stages are:

- reinstitution and/or change of antibiotic therapy.

- insertion of drain with antibiotic therapy.

- partial or complete debridement of the graft material.

- total debridement of the graft and sinus cavity by oral approach and/or functional endoscopic sinus surgery.

Without a microbiological assay, immediate therapy is directed toward the most common pathogens and the common resistant strains of bacteria. The antibiotics chosen should be able to achieve high tissue concentrations and have the broadest spectrum possible. If signs of infection are noted, it may be appropriate to change from the antibiotic used in prophylaxis to one with a wider spectrum (Augmentin or Levaquin). Metronidazole, a member of the nitroimidazole group, may be included for its bactericidal effect against gram-positive and gram-negative anaerobic bacteria. It must be used with an additional antibiotic (Augmentin or Levaquin) that is effective against facultative bacteria. A culture can be taken to obtain information in case the infection is resistant to the chosen antibiotic. In many instances, however, it is difficult to obtain a culture that is not contaminated by oral bacteria or to obtain results in a reasonable amount of time.

If a Penrose drain is placed (Figure 35), it is best, if possible, to place it in a location that is not directly over the graft. Placing the drain through an incision over the window and graft site may increase the potential for an oroantral fistula. Figure 35 shows the placement of a Penrose drain in an existing fistula. The drain was left in place for 3 days, and after removal, the infection resolved.

If the infection does not respond to either of the above therapies, debridement of the infected graft material may be the only remaining means of infection control. All graft material can be removed followed by thorough flushing of the subantral space. Regrafting at the time of debridement is an option when signs of infection are minimal, but the risk of reinfection may be increased. It is usually advisable to wait until symptoms disappear before retreatment. In cases of late infections an alternative therapy of partial debridement may be considered. Attempting to remove the hard "shell" surrounding the infected central portion of the graft will probably result in destruction of the surrounding Schneiderian membrane. To avoid this negative consequence, it may be advisable to leave the "shell" in place after thorough debridement and irrigation of the central portion of the infected graft. Urban et al ${ }^{106}$ have reported on this technique with successful results in 8 patients. The collagen membrane and discolored/infected graft materials are removed, the cavity is rinsed with saline, treated with doxycycline putty for 2 minutes, rinsed again, and closed without additional grafting. Implant placement and regrafting of the central
TA B LE 6 Clinical recommendations to limit intraoperative and postoperative complications

List of clinical recommendations

1. Careful assessment of the medical history of the patient

2. Preoperative computed tomogrpahy scan to evaluate sinus anatomy and identify preexisting pathology

3. Proper patient selection stressing a healthy maxillary sinus

4. A smoking-cessation protocol is always recommended and, especially in the case of heavy smokers ( $\geq 15$ cigarettes a day), this is evaluated with caution

5. Resolution of periodontal and endodontic diseases

6. Adequate antibiotic prophylaxis

7. Achieve full-mouth plaque score and full-mouth bleeding score of $<15 \%$. In the case of provisional crowns, it is advisable to remove the temporary crowns and disinfect the abutments with antiseptic solution

8. Preoperative disinfection of the skin with an antiseptic solution and mouthrinses with chlorhexidine

9. Use of sterile draping and infection-control protocol

10. Keep the incisions distant from the antrostomy

11. Prevent salivary contamination of bone graft and/or other biomaterials

12. Intra- and postoperative control of the hemostasis

13. Prevention of bone overheating

14. Use of 2 different sets of surgical instruments: 1 for the flapelevation phase and the other for the grafting phase

15. Rinsing the surgical field with sterile saline solution

16. Keeping the surgical time as short as possible

17. Postoperative chlorhexidine rinses

18. Correct postoperative pharmacological therapy

19. Preplanned check-ups: weekly for the first month and monthly for the following $3 \mathrm{mo}$

part of the graft can generally commence with only a minor change to the original schedule. The retained portion of the graft matures and allows for implant placement and stability. Histology showing vital bone in this nongrafted repair location has been presented by Khouly et al $^{107}$

Best clinical practices:

- ensure proper case selection, proper prophylactic antibiotics, and infection-control surgical protocol.

- treat early if infection suspected.

- change the treatment if no response within 7 days.

\section{7 | POSTOPERATIVE SINUSITIS}

\section{1 | Etiology}

By decreasing the size of the sinus by grafting the floor, maxillary sinus floor elevation has the potential to create more favorable sinus 
drainage. Many clinicians have noticed that patients who presented with a history of low-grade chronic sinusitis before sinus elevation surgery were less susceptible to this condition after that surgery was performed. This is a result both of the decreased volume of the sinus and the fact that the sinus floor is now closer to the ostium, or point of drainage. This assumes that a proper membrane elevation that extends up the medial wall has raised the floor without creating a narrow, difficult to drain, crestal extension of the sinus floor against the medial wall. Generally, one should expect to see a short-term increase in membrane thickness caused by a postsurgical inflammatory response. This would appear to be temporary, as a study by Peleg et al, ${ }^{108}$ of follow-up evaluations of 24 sinus grafts, revealed that 12 membranes decreased in size, 11 remained the same, and 1 increased in size.

Sinusitis after sinus elevation surgery, which has been reported in $3 \%-20 \%$ of cases, ${ }^{71}$ is generally mild in nature. Symptoms may include mild discomfort, stuffiness, and difficulty in breathing. A moderate-to-severe postoperative sinusitis is most likely to be a result of blockage of osteomeatal drainage owing to inflammation and/or sinus infection. The various etiologies of sinusitis include:

- postsurgical inflammatory changes.

- bleeding into the sinus after membrane perforation.

- bacterial contamination/infection after membrane perforation.

- blockage of the osteomeatal complex as a result of:

o intrasinus bleeding

- Graft material lost through perforation

- Elevation of large cysts or thickened membranes to the level of the ostium.

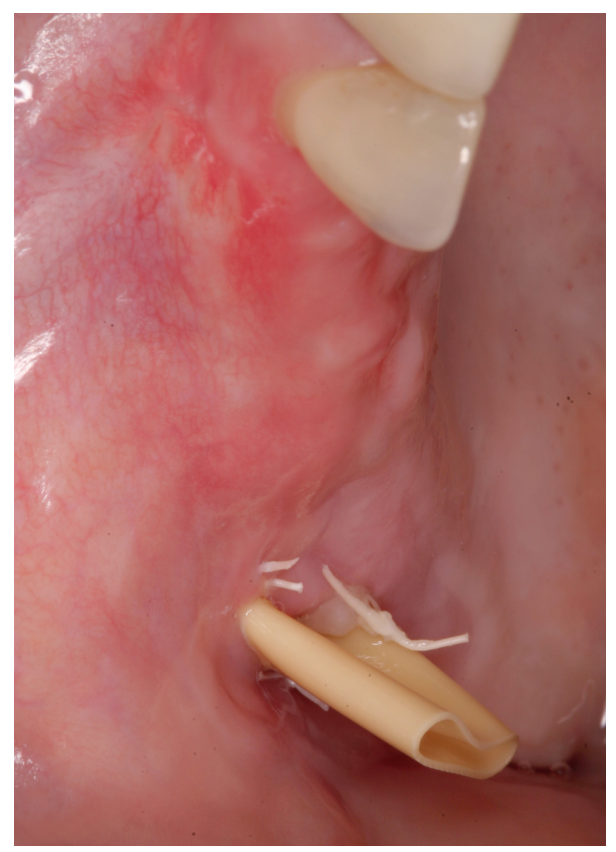

FIGURE 35 Penrose drain placed in existing fistula

\section{2 | Prevention}

Prevention of postoperative sinusitis begins with an evaluation of patient medical history and final case selection. Patients with a previous history of inflammatory sinus disease are more likely to have a postoperative sinusitis than patients with a negative history of this disease. ${ }^{5,27}$ Preoperative sinus pathology should be evaluated by computed tomography and potential problems possibly addressed before sinus elevation surgery. The proper presurgical protocol thus may include:

- 3-dimensional treatment planning to discover preexisting pathology.

- prior treatment of inflammatory disease by antibiotic and anti-inflammatory medications.

- resolution of pathology by functional endoscopic sinus surgery.

A protocol suggested by Torretta et $\mathrm{al}^{28}$ recommends that all preexisting reversible sinus conditions be addressed prior to sinus elevation surgery. If the problem is caused by potential or actual blocked drainage, positive therapy might involve endoscopic marsupialization of a mucous retention cyst, removal of polyps or thickened membranes, or surgical widening of the ostium via functional endoscopic sinus surgery. If perforation of the sinus membrane occurs during elevation surgery, it must be repaired in a manner which ensures that the repair membrane is stable and prevents particulate graft material from escaping into the sinus cavity, as this may be a nidus for inflammatory changes, infection, or blockage of the ostium. As will be seen, treatment for sinus infections may become more complex if the integrity of the Schneiderian membrane is lost.

\section{3 | Treatment}

Many clinicians routinely prescribe decongestants, such as oxymetazoline (Afrin), for postoperative use. The postoperative incidence of patients requiring this therapy is so infrequent that many clinicians do not include this in their usual postoperative protocol but prescribe it on an as-needed basis. Nasal lavage with sterile saline rinses can be used as adjunctive therapy. Treatment will depend on the severity and presumptive etiology of the sinusitis. A mild sinusitis may respond to decongestants. If the etiology is a combination of inflammation and infection, combined antibiotic and anti-inflammatory therapy may be effective. If there is no resolution, and the situation involves infection in both the graft and the sinus, therapy may involve a surgical approach orally and/or via endoscopic surgery. As deliberated in the discussion above and the summary below, treatment may become more complex if containment of the graft material is lost. Two therapeutic protocols should be considered. ${ }^{109}$

Best clinical practice includes:

- if the graft is well contained under the Schneiderian membrane but signs and symptoms still persist after an additional pharmacological regimen (usually 7 additional days), partial or total removal of the bone graft by oral access combined with additional 

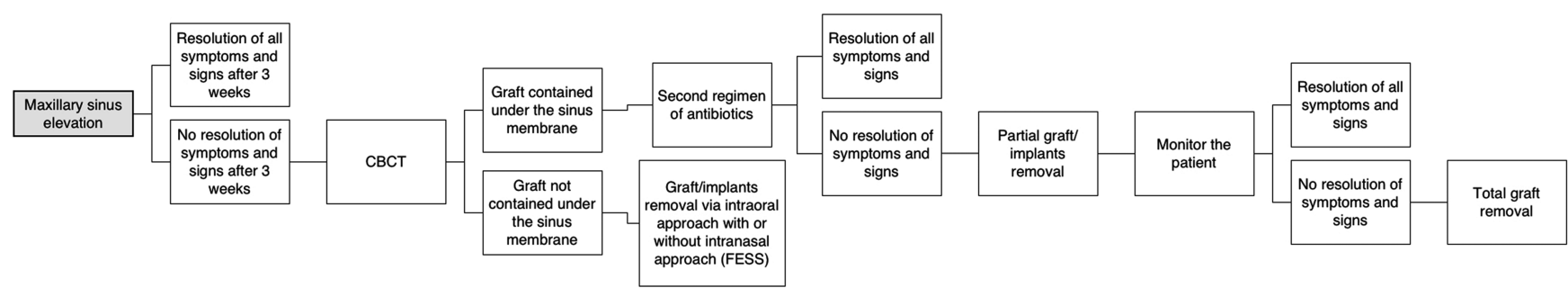

FIGURE 36 Algorithm for treatment of sinus graft infections, sinus infections, and postoperative sinusitis

pharmacological therapy is recommended.

- if the graft is not contained under the sinus membrane and loss of graft material into the sinus is present (as seen on computed tomography), and symptoms still persist after extended antibiotic therapy (usually 7 additional days), a multidisciplinary approach to manage the complication is mandatory.

The above information can be utilized to create an algorithm for the treatment of sinus graft infections, sinus infections, and postoperative sinusitis (Figure 36).

It is apparent in the algorithm that if symptoms occur early or remain after a time interval of 3 weeks, a cone-beam computed tomography scan should be taken for diagnostic purposes. The results of the scan determine a pathway for treatment that is directed by containment or lack of containment of the graft material. If the graft material is not contained, graft removal using an intraoral approach, with or without functional endoscopic sinus surgery, is usually required. If the implants do not have sufficient bone support following loss of the graft, they will also be removed. In cases where the graft material is contained (sinus graft infection), the therapeutic course is determined by the patient's response to a series of increasingly invasive attempts to resolve the infection. In order, they consist of a second round of antibiotics, partial graft/implants removal, and complete graft removal if there is no resolution. Infections of the maxillary sinus can have quite severe adverse outcomes well beyond loss of the graft material. Pan sinusitis, intraorbital abscesses with possible loss of sight, and intracranial abscesses have been mentioned in this chapter. The greatest errors that one can make in these cases are waiting too long to begin therapy, waiting too long before changing an ineffective therapy, and not taking advantage of the knowledge and skills of other surgical specialists by referral.

\section{4 | Other postoperative complications}

\subsection{1 | Loss of graft material through the surgical window}

An increase in intrasinus pressure, which may be caused by postoperative inflammation or bleeding from within the sinus, can result in loss of graft material through the window (Figure 37A,B). This is likely to occur if a membrane was not placed over the window or if the membrane was not properly stabilized. The displaced graft material is likely to cause an elevation in the buccal mucosa, which can be removed with a small flap entry (not over the window or membrane) or left in place and addressed at the time of implant placement. Some clinicians stabilize bioabsorbable barrier membranes with resorbable tacks or a mattress suture. The incidence of this complication is quite low, making routine mechanical membrane stabilization unnecessary. It would be appropriate, however, to choose a membrane

A

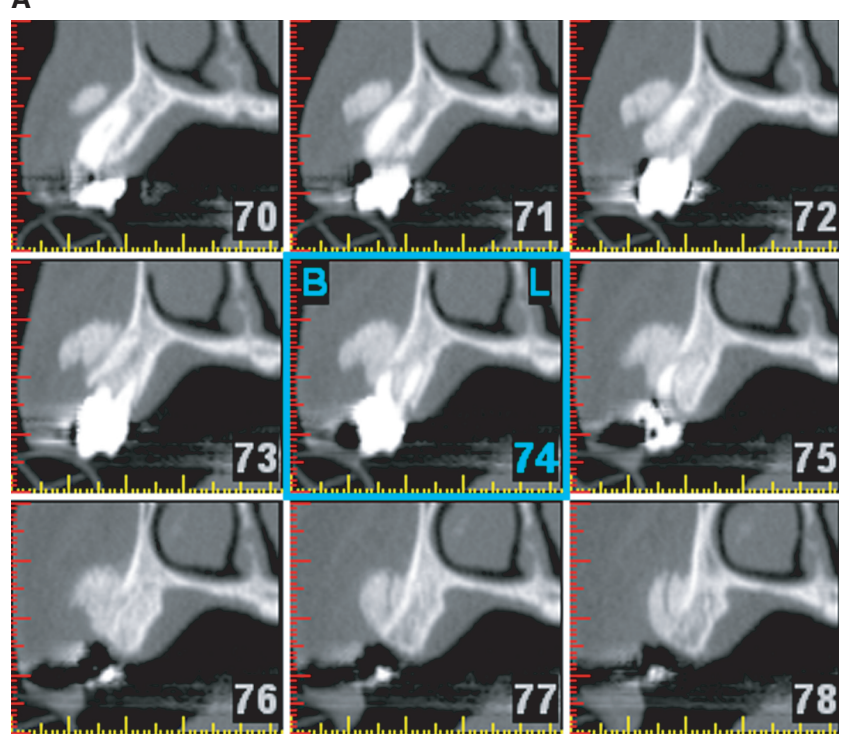

B

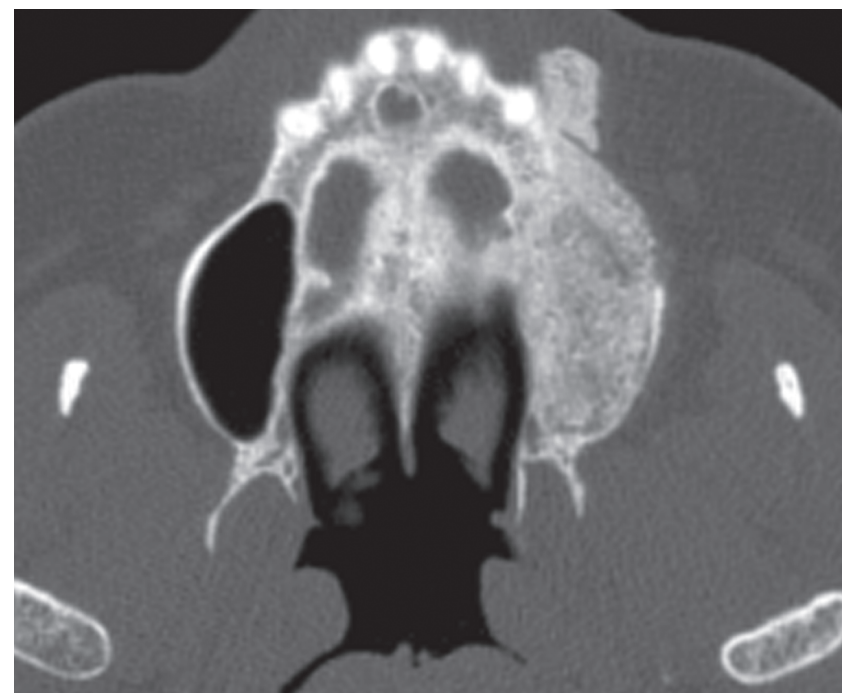

FIGURE 37 (A) Displaced graft material following intrasinus bleeding (cross-sectional view). (B) Displaced graft material following intrasinus bleeding (axial computed tomography view) 

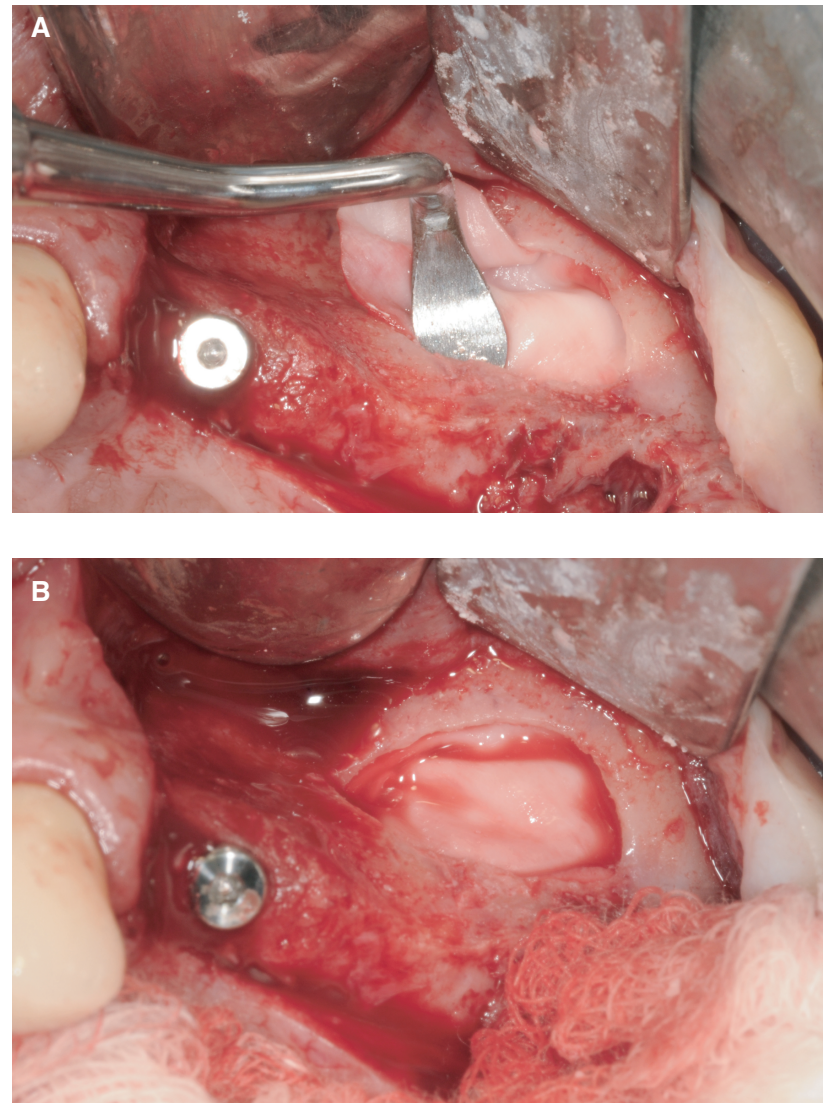

FIGURE 38 (A) The membrane is tucked between the inner bony walls and the graft material. Instruments for sinus elevation are useful for this procedure. (B) Che collagen membrane is completely reflected inside the antrostomy

that is flexible when wet so that it may conform to the shape of the lateral wall. An alternate method of stabilizing the repair membrane is to place it immediately within the sinus window, over the graft, extending approximately $2 \mathrm{~mm}$ in each direction for stabilization ${ }^{110}$ (Figure 38A,B).

\section{5 | Migration of implants into sinus or sinus graft}

This complication was more common when cylindrical implants were used in the posterior maxilla ${ }^{111}$ and it is still seen with screw-form implants when biologic boundaries are pushed to or beyond the limit (Figure 39). The problem is usually caused by an initially inadequate or early loss of primary stability. It can also be caused by the loss of supporting bone owing to infection. Many clinicians reserve simultaneous implant placement for those patients with a minimum of 4-5 $\mathrm{mm}$ of crestal bone. While simultaneous placement has been reported to be successful in 1-2 mm of crestal bone, ${ }^{108}$ one must consider the potential risk. If an implant is placed in 1-3 $\mathrm{mm}$ of $\mathrm{cr}-$ estal bone and primary closure is not achieved and maintained, the early formation of the biologic width may remove more than half of the supporting bone well before the graft has matured and become supportive.

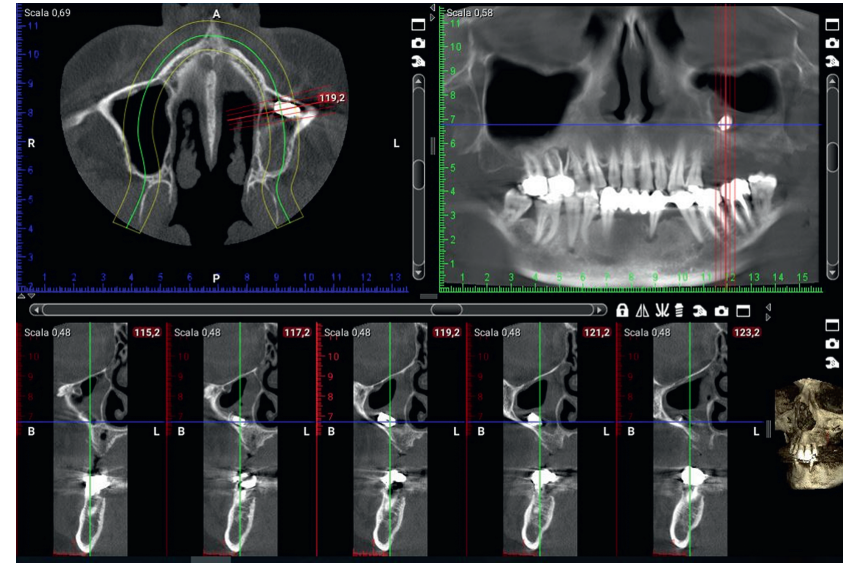

FIGURE 39 Migration of implant into the sinus cavity

\section{8 | CONCLUSIONS}

The maxillary sinus elevation procedure using a lateral window approach has been shown to be the most successful bone augmentation procedure that is performed as a pre-prosthetic procedure before implant placement. ${ }^{99}$ When success is measured according to patient outcome (ie, success of the grafting procedure) the high success rate achieved is due to the fact that complications are minimal and can be reduced through proper case selection, proper preventive antibiotic selection, good surgical technique, and proper and prompt handling of intraoperative and postoperative complications when they occur. Properly performed sinus grafting does not alter proper sinus function ${ }^{5}$ and does not alter the characteristics of the voice. ${ }^{112}$ When success is measured according to implant outcome (ie, implant survival rate) it has been shown that implant survival rates in the high 90th percentile can be achieved through proper decision making with regard to implant surfaces (textured), graft materials (highest survival with xenografts), and the placement of a barrier membrane over the window. ${ }^{99,112-115}$ In this chapter, each discussion regarding prevention of an intraoperative or a postoperative complication advised that a computed tomography scan analysis should be obtained preoperatively. The authors believe that this should be considered as part of the universal standard of care. It is believed, by most clinicians, that the information obtained relative to sinus health and sinus anatomy is a key factor in reducing complications to a minimum.

\section{REFERENCES}

1. Mc Gowan DA, Baxter PW, James J. The maxillary sinus and its dental implications. Oxford,UK: Wright, Butterworth-Heinemann Ltd; 1993.

2. Cawood JI, Howell RA. A classification of the edentulous jaws. Int J Oral Maxillofac Surg. 1988;17:232-236.

3. Cawood JI, Howell RA. Reconstructive preprosthetic surgery. I. Anatomical considerations. Int J Oral Maxillofac Surg 1991;20:75-82.

4. Garg AK. Augmentation grafting of the maxillary sinus for the placement of dental implants: anatomy, physiology, and procedure. Implant Dent. 1999;8:36-46. 
5. Timmenga NM, Raghoebar GM, Boering G, van Weissenbruch R. Maxillary sinus function after sinus lifts for the insertion of dental implants. J Oral Maxillofac Surg. 1997;55:936-939.

6. Tatum H. Alabama Implant Congress, Birmingham, AL:1976.

7. Boyne PJ, James RA. Grafting of the maxillary sinus floor with autogenous marrow and bone. J Oral Surg. 1980;38:613-616.

8. Wallace SS, Tarnow DP, Froum SJ, et al. Maxillary sinus elevation by lateral window approach: evolution of technology and technique. J Evid Based Dent Pract. 2012;12(Suppl 3):161-171.

9. Taschieri S, Testori T, Corbella S, et al. Platelet-Rich Plasma and Deproteinized Bovine Bone Matrix in Maxillary Sinus Lift Surgery: a Split-Mouth Histomorphometric Evaluation. Implant Dent. 2015;24:592-597.

10. Stammberger $\mathrm{H}$. Functional endoscopic sinus surgery: the Messerklinger technique. Philadelphia, PA: BC Decker; 1991.

11. Pommer B, Unger E, Sütö D, Hack N, Watzek G. Mechanical properties of the Schneiderian membrane in vitro. Clin Oral Impl Res. 2009;20:633-637.

12. Insua A, Monje A, Chan HL, Zimmo N, Shaikh L, Wang HL. Accuracy of Schneiderian membrane thickness: a cone-beam computed tomography analysis with histological validation. Clin Oral Implant Res. 2016;28:654-661.

13. Van den Bergh JP, ten Bruggenkate CM, Disch FJ, Tuinzing DB. Anatomical aspects of sinus floor elevations. Clin Oral Implants Res. 2000;11:256-265.

14. Underwood AS. An inquiry into the anatomy and pathology of the maxillary sinus. J Anat Physiol. 1910;44:354-369.

15. Krenmair G, Ulm CW, Lugmayr H. Maxillary sinus septa: incidence, morphology and clinical implications. J Craniomaxillofac Surg. 1997;25:261-265.

16. Kim MJ, Jung UW, Kim CS, et al. Maxillary sinus septa: prevalence, height, location, and morphology. A reformatted computed tomography scan analysis. J Periodontol 2006;77:903-908.

17. Rosano G, Taschieri S, Gaudy JF, Lesmes D, Del Fabbro M. Maxillary Sinus Septa: a Cadaveric Study. J Oral Maxillofac Surg. 2010;68:1360-1364.

18. Velasquez-Plata D, Hovey L, Peach CC, Alder ME. Maxillary sinus septa: a 3-dimensional computerized tomographic scan analysis. Int J Oral Maxillofac Implants. 2002;17:854-860.

19. Gaudy JF. Anatomie Clinique. Editions CdP Groupe Liaisons, 2003.

20. Rosano G, Taschieri S, Gaudy JF, Del Fabbro M. Maxillary sinus vascularization: a cadaveric study. J Craniofac Surg. 2009;20:940-943.

21. Solar P, Geyerhofer U, Traxler H, Windisch A, Ulm C, Watzek G. Blood supply to the maxillary sinus relevant to sinus floor elevation procedures. Clin Oral Implants Res. 1999;10:34-44.

22. Traxler H, Windisch A, Geyerhofer U, Surd R, Solar P, Firbas W. Arterial blood supply of the maxillary sinus. Clin Anat. 1999;1:417-421.

23. Elian N, Wallace S, Cho SC, Jalbout ZN, Froum S. Distribution of the maxillary artery as it relates to sinus floor augmentation. Int $J$ Oral Maxillofac Implants. 2005;20:784-787.

24. Mardinger O, Abba M, Hirshberg A, Schwartz-Arad D. Prevalence, diameter and course of the maxillary intraosseous vascular canal with relation to sinus augmentation procedure: a radiographic study. Int J Oral Maxillofac Surg. 2007;3:735-738.

25. Testori T, Rosano G, Taschieri S, Del Fabbro M. Ligation of an unusually large vessel during maxillary sinus floor augmentation. A case report. Eur J Oral Implantol 2010;3:255-258.

26. Lekholm U, Zarb GA. Patient selection. In: Branemark PI, Zarb GA, Albrektsson T. Tissue Integrated Prosthesis. Osseointegration in Clinical Dentistry. Chicago: Quintessence; 1985: 199-209.

27. Timmenga NM, Raghoebar GM, Liem RS, van Weissenbruch R, Manson WL, Vissink A. Effects of maxillary sinus floor elevation surgery on maxillary sinus physiology. Eur J Oral Sci. 2003;111:189-197.

28. Torretta S, Mantovani M, Testori T, Cappadona M, Pignataro L. Importance of ENT assessment in stratifying candidates for sinus floor elevation: a prospective clinical study. Clin Oral Implants Res 2013;24(Suppl A100):57-62.

29. Carmeli G, Artzi Z, Kozlovsky A, Segev Y, Landsberg R. Antral computerized tomography pre-operative evaluation: relationship between mucosal thickening and maxillary sinus function. Clin Oral Impl Res. 2011;22:78-82.

30. Friedland B, Metson R. A guide to recognizing maxillary sinus pathology and for deciding on further preoperative assessment prior to maxillary sinus augmentation. Int J Periodontics Restorative Dent. 2014;34:807-815.

31. Hernández-Alfaro F, Torradeflot MM, Marti C. Prevalence and management of Schneiderian membrane perforations during sinus-lift procedures. Clin Oral Implants Res. 2008;19:91-98.

32. Som M, Brandwein M. Sinonasal cavities: In ammatory diseases, tumors, fractures, and postoperative findings. In: Som PM, Curtin HD, eds. Head and Neck Imaging, 3rd edn. St. Louis: Mosby; 1996:126-315.

33. Pignataro L, Mantovani M, Torretta S, Felisati G, Sambataro G. ENT assessment in the integrated management of candidate for (maxillary) sinus lift. Acta Otorhinolaryngol Ital. 2008;28:110-119.

34. Mantovani M. Othorhinolayngolocical implications in augmentation of the maxillary sinus. In: Testori T, Del Fabbro M, Weinstein R, Wallace S. Maxillary sinus surgery. Chicago, IL: Quintessence; 2009

35. Lopatin AS, Sysolyatin SP, Sysolyatin PG, Melnikov MN. Chronic maxillary sinusitis of dental origin: is external surgical approach mandatory? Laryngoscope. 2002;112:1056-1059.

36. Mehra P, Murad H. Maxillary sinus disease of odontogenic origin. Otolaryngol Clin North Am. 2004;37:347-364.

37. Albu S, Baciut M. Failures in endo- scopic surgery of the maxillary sinus. Otolaryngol Head Neck Surg. 2010;142:196-201.

38. Melen I, Lindahl L, Andreasson L, Rundcrantz H. Chronic maxillary sinusitis. Definition, diagnosis and relation to dental infections and nasal polyposis. Acta Otolaryngol 1986;101:320-327.

39. Longhini $A B$, Branstetter BF, Ferguson BJ. Otolaryngologists' perceptions of odontogenic maxillary sinusitis. Laryngoscope. 2012;122:1910-1914.

40. Saibene AM, Vassena C, Pipolo C, et al. Odontogenic and rhinogenic chronic sinusitis: a modern microbiological comparison. Int Forum Allergy Rhinol. 2016;6:41-45.

41. Taschieri S, Torretta S, Corbella S, et al. Pathophysiology of sinusitis of odontogenic origin. J Investig Clin Dent 2015;8:1-7.

42. Aruni AW, Dou Y, Mishra A, Fletcher HM. The biofilm communityrebels with a cause. Curr Oral Health Rep. 2015;2:48-56.

43. Sunde PT, Olsen I, Debelian GJ, Tronstad L. Microbiota of periapical lesions refractory to endodontic therapy. J Endod. 2002;28:304-310.

44. Hunsaker DH, Leid JG. The relationship of biofilms to chronic rhinosinusitis. Curr Opin Otolaryngol Head Neck Surg. 2008;16:237-241.

45. Perloff JR, Palmer JN. Evidence of bacterial biofilms in a rabbit model of sinusitis. Am J Rhinol. 2005;19:1-6.

46. Perloff JR, Palmer JN. Evidence of bacterial biofilms on frontal recess stents in patients with chronic rhinosinusitis. Am J Rhinol. 2004;18:377-380.

47. Ramadan HH, Sanclement JA, Thomas JG. Chronic rhinosinusitis and biofilms. Otolaryngol Head Neck Surg. 2005;132:414-417.

48. Sanclement JA, Webster P, Thomas J, Ramadan HH. Bacterial biofilms in surgical specimens of patients with chronic rhinosinusitis. Laryngoscope. 2005;115:578-582. 
49. Foreman A, Psaltis AJ, Tan LW, Wormald PJ. Characterization of bacterial and fungal biofilms in chronic rhinos- inusitis. Am J Rhinol Allergy. 2009;23:556-561.

50. Healy DY, Leid JG, Sanderson AR, Hunsaker DH. Biofilms with fungi in chronic rhinosinusitis. Otolaryngol Head Neck Surg. 2008;138:641-647.

51. Chole RA, Faddis BT. Anatomical evidence of microbial biofilms in tonsillar tissue: a possible mechanism to explain chronicity. Arch Otolaryngol Head Neck Surg. 2003;129:634-636.

52. Troeltzsch M, Pache C, Troeltzsch M, et al. Etiology and clinical characteristics of symptomatic unilateral maxillary sinusitis: a review of 174 cases. J Craniomaxillofac Surg. 2015;43:1522-1529.

53. Blanksma CJ, Brand HS. Cocaine abuse: orofacial manifestations and implications for dental treatment. Int Dent J. 2005;55: 365-369.

54. Silvestre FJ, Perez-Herbera A, Puente-Sandoval A, Bagán JV. Hard palate perforation in cocaine abusers: a systematic review. Clin Oral Investig. 2010;14:621-628.

55. Heitz-Mayfield LJ, Huynh-Ba G. History of treated periodontitis and smoking as risks for implant therapy. Int J Oral Maxillofac Implants. 2009;24(Suppl):39-68.

56. Testori T, Weinstein RL, Taschieri S, Del Fabbro M. Risk factor analysis following maxillary sinus augmentation: a retrospective multicenter study. Int J Oral Maxillofac Implants. 2012;27:1170-1176.

57. Flanagan D. Arterial supply of maxillary sinus and potential for bleeding complication during lateral approach sinus elevation. Implant Dent. 2005;14:336-338.

58. Vercellotti T, De Paoli S, Nevins M. The piezoelectric bony window osteotomy and sinus membrane elevation: introduction of a new technique for simplification of the sinus augmentation procedure. Int J Periodontics Restorative Dent. 2001;21:561-567.

59. Beziat JL, Vercellotti T, Gleizal A. What is Piezosurgery? Twoyears' experience in craniomaxillofacial surgery. Rev Stomatol Chir Maxillofac. 2007;108:101-107.

60. Geha HJ, Gleizal AM, Beziat JL. Sensitivity of the inferior lip and chin following mandibular bilateral sagittal split osteotomy using Piezosurgery. Plast Reconstr Surg. 2006;118:598-607.

61. Atieh MA, Alsa Beeha NH, Tawse-Smith A, Faggion CM Jr, Duncan WJ. Piezoelectric surgery vs rotary instruments for lateral maxillary sinus floor elevation: a systematic review of and meta- analysis of intra- and postoperative complications. Int J Oral Maxillofac Implants. 2015;30:1262-1271.

62. Zijderveld SA, van den Bergh JPA, Schulten EAJM, ten Bruggenkate CM. Anatomical and surgical findings and complications in 100 consecutive maxillary sinus floor elevations. J Oral Maxillofac Surg. 2008; $66: 1426-1438$

63. Schwartz-Arad D, Herzberg R, Dolev E. The prevalence of surgical complications of the sinus graft procedure and their impact on implant survival. J Periodontol. 2004;75:511-516.

64. Kasabah S, Krug J, Simunek A, Lecaro MC. Can we predict maxillary sinus mucosa perforation? Acta Med. 2003;46:19-23.

65. Cho S-C, Wallace SS, Froum SJ, Tarnow DP. Influence of anatomy on Schneiderian membrane perforations during sinus elevation surgery: three-dimensional analysis. Pract Proced Aesthet Dent. 2001:13:160-163.

66. Chan HL, Monje A, Suarez F, Benavides E, Wang HL. Palatonasal recess on medial wall of the maxillary sinus and clinical implications for sinus augmentation via lateral window approach. J Periodontol. 2013;84:1087-1093.

67. Avila-Ortiz G, Wang HL, Galindo-Moreno P, Misch CE, Rudek I, Neiva R. Influence of lateral window dimensions on vital bone formation following maxillary sinus augmentation. Int J Oral Maxillofac Implants. 2012;27:1230-1238.

68. Wallace SS, Mazor Z, Froum SJ, Cho SC, Tarnow DP. Schneiderian membrane perforation rate during sinus elevation using
Piezosurgery: clinical results of 100 consecutive cases. Int Periodontics Restorative Dent. 2007;27:413-419.

69. Blus C, Szmukler-Moncler S, Salama M, Salama H, Garber D. Sinus bone grafting procedures using ultrasonic bone surgery: 5-year experience. Int J Periodontics Restorative Dent. 2008;28:221-229.

70. Toscano NJ, Holtzclaw D, Rosen PS. The effect of piezoelectric use on open sinus lift perforation: a retrospective evaluation of 56 consecutively treated cases from private practices. J Periodontol. 2010;81:167-171.

71. Barone A, Santini S, Marconcini S, Giacomelli L, Gherlone E, Covani $\mathrm{U}$. Osteotomy and membrane elevation during the maxillary sinus augmentation procedure. A comparative study: piezoelectric device vs. conventional rotary instruments. Clin Oral Implants Res 2008;19:511-515.

72. Lozada JL, Goodacre C, Al-Ardah AJ, Garbacea A. Lateral and crestal bone planing antrostomy: a simplified surgical procedure to reduce the incidence of membrane perforation during maxillary sinus augmentation procedures. J Prosthet Dent. 2011;105:147-153.

73. Nishimoto N, Kan J, Lozada JL, Wallace SS, Rungcharassaeng KR. Incidence of maxillary sinus membrane perforation during lateral window approach using the DASK technique (lateral bone-planing antrostomy). Manuscript in preparation

74. Keller EE, Eckert SE, Tolman DE. Maxillary antral and nasal onestage inlay composite bone graft. Preliminary report on 30 recipient sites. J Oral Maxillofac Surg 1994;52:438-447.

75. Triplett RG, Schow SR. Autologous bone grafts and endosseous implants: complementary techniques. J Oral Maxillofac Surg. 1996;54:486-494.

76. Proussaefs P, Lozada J, Kim J, Rohrer MD. Repair of the perforated sinus membrane with a resorbable collagen membrane: a human study. Int J Oral Maxillofac Implants. 2004;19:413-420.

77. Fugazzotto PA, Vlassis JM. A simplified classification and repair system for sinus membrane perforations. J Periodontol. 2003;74:1534-1541

78. Pikos MA. Maxillary sinus repair: report of a technique for large perforations. Implant Dent. 1999;8:29-33.

79. Proussaefs A, Lozada J. The, "Loma Linda pouch": a technique for repairing the perforated sinus membrane. Int J Periodontics Restorative Dent. 2003;23:593-597.

80. Shlomi B, Horowitz I, Kahn A, Dodriyan A, Chaushu G. The effect of sinus membrane perforation and repair with Lambone sheet on the outcome of maxillary sinus floor augmentation: a radiographic assessment. Int J Oral Maxillofac Implants. 2004;19:559-562.

81. Testori T, Wallace SS, Del Fabbro M, et al. Repair of large sinus membrane perforations using stabilized collagen barrier membranes: surgical techniques with histologic, and radiographic evidence of success. Int J Periodontics Restorative Dent. 2008;28:9-17.

82. Vlassis JM, Fugazzotto PA. A classification system for sinus membrane perforations during augmentation procedures with options for repair. J Periodontol. 1999;70:692-699.

83. Haas R, Baron M, Donath K, Zechner W, Watzek G. Porous hydroxyapatite for grafting the maxillary sinus. Int J Oral Maxillofac Implants. 2002;17:337-346.

84. Hürzeler MB, Quiñones $C R$, Kirsch A, Gloker C, Schüpbach P, Strub JR. Maxillary sinus augmentation using different grafting materials and dental implants in monkeys. Part 1. Evaluation of anorganic bovine bone-derived bone matrix. Clin Oral Implants Res 1997;8:476-486

85. Jensen OT, Shulman LB, Block MS, lacono VJ. Report of the Sinus Consensus Conference of 1996. Int J Oral Maxillofac Implants. 1998:13(Suppl):11-45.

86. Khoury F. Augmentation of the sinus floor with mandibular bone block and simultaneous implantation. Int J Oral Maxillofac Implants. 1999;14:557-564. 
87. Ardekian L, Oved-Peleg E, Mactei EE, Peled M. The clinical significance of sinus membrane perforation during augmentation of the maxillary sinus. J Oral Maxillofac Surg. 2006;64:277-282.

88. Karabuda C, Arisan V, Hakan O. Effects of sinus membrane perforation on the success of dental implants placed in the augmented sinus. J Periodontol. 2006;77:991-997.

89. Froum SJ, Khouly I, Favero G, Cho SC. Effect of maxillary sinus membrane perforation on vital bone formation and implant survival: a retrospective study. J Periodontol. 2013;84:1094-1099.

90. Dohan Ehrenfest DM, Rasmusson L, Albrektsson T. Classification of platelet concentrates: from pure platelet-rich plasma (P-PRP) to leucocyte- and platelet-rich fibrin (L-PRF). Trends Biotechnol. 2009;27:158-167.

91. Dohan Ehrenfest DM. How to optimize the preparation of leukocyte- and platelet-rich fibrin (L-PRF, Choukroun's technique) clots and membranes: introducing the PRF Box. Oral Surg Oral Med Oral Pathol Oral Radiol Endod. 2010;110:275-278.

92. Simonpieri A, Choukroun J, Del Corso M, Sammartino G, Dohan Ehrenfest DM. Simultaneous sinus-lift and implantation using microthreaded implants and leukocyte- and platelet-rich fibrin as sole grafting material: a six-year experience. Implant Dent. 2011;20:2-12.

93. Maestre-Ferrín L, Galán-Gil S, Carrillo-García C, PeñarrochaDiago M. Radiographic findings in the maxillary sinus: comparison of panoramic radiography with computed tomography. Int J Oral Maxillofac Implants. 2011;26:341-346.

94. Hadar T, Shvero J, Nageris BI, Yaniv E. Mucus retention cyst of the maxillary sinus: the endoscopic approach. Br J Oral Maxillofac Surg 2000;38:227-229.

95. Testori T, Mantovani M, Wallace SS, et al. Maxillary sinus elevation with simultaneous cyst deflation: a clinical prospective study. Int J Periodontics Restorative Dent. 2015. Manuscript in preparation.

96. Lockhart R, Ceccaidi J, Bertrand JC. Postoperative maxillary cyst following sinus bone graft: report of a case. Int J Oral Maxillofac Implants. 2000;15:583-586.

97. Misch CM, Misch CE, Resnik RR, Ismael YH, Appel B. Post-operative maxillary cyst associated with a maxillary sinus elevation procedure: a case report. J Oral Implantol. 1991;17:432-437.

98. Felisati G, Lozza P, Chiapasco M, Borloni R. Endoscopic removal of an unusual foreign body in the sphenoid sinus: an oral implant. Clin Oral Implants Res. 2007 Dec;18:776-780.

99. Aghaloo TL, Moy PK. Which hard tissue augmentation techniques are the most successful in furnishing bony support for implant placement? Int J Oral Maxillofac Implants. 2007;22(Suppl):49-70.

100. Misch CE. Contemporary implant dentistry, 3rd edn. St Louis, MO: Mosby; 2008:905-974.

101. Barone A, Santini S, Sbordone L, Crespi R, Covani U. A clinical study of the outcomes and complications associated with maxillary sinus augmentation. Int J Oral Maxillofac Implants. 2006;21:81-85.

102. Levin L, Herzberg R, Dolev E, Schwartz-Arad D. Smoking and complications of onlay bone grafts and sinus lift operations. Int J Oral Maxillofac Implants. 2004;19:369-373.

103. Parmar C, Meda KP. Achilles tendon rupture associated with combination therapy of levofloxacin and steroid in four patients and a review of the literature. Foot Ankle Int. 2007;28:1287-1289.
104. Stephenson AL, Wu W, Cortes D, Rochon PA. Tendon injury and fluoroquinolone use: a systematic review. drug saf. 2013;36:709-721.

105. Wagenberg B, Froum SJ. A retrospective of 1925 consecutively placed implants from 1988-2004. J Oral Maxillofac Implants. 2006;21:565-578.

106. Urban I, Nagursky H, Church C, Lozada JL. Incidence, diagnosis, and treatment of sinus graft infection after sinus floor elevation: a clinical study. Int J Oral Maxillofac Implants. 2012;27:449-457.

107. Khouly I, Phelan J, Munoz C, Froum SJ. Human histologic and radiographic evidence of bone formation in a previously infected maxillary sinus graft following debridement without re-grafting; a case report. Int J Perio Restorative Dent. 2016;36:723-729.

108. Peleg M, Garg AK, Mazor Z. Predictability of simultaneous implant placement in the severely atrophic posterior maxilla: a 9-year longitudinal experience study of 2132 implants placed into 731 human sinus grafts. Int J Oral Maxillofac Implants. 2006;21:94-102.

109. Testori T, Drago L, Wallace SS, et al. Prevention and treatment of postoperative infections after maxillary sinus elevation surgery: clinical consensus and recommendations. Int J Dent. 2012;2012:365809.

110. Testori T, Mandelli F, Valentini P, Wallace S. A novel technique to prevent the loss of graft material through the antrostomy after sinus surgery: a technical note. Int J Oral Maxillofac Implants. 2014;29:e272-e274.

111. Regev E, Smith RA, Perrott DH, Pogrel MA. Maxillary sinus complications related to endosseous implants. Int J Oral Maxillofac Implants. 1995;10:451-461.

112. Tepper G, Haas R, Schneider B, et al. Effects of sinus lifting on voice quality. A prospective study and risk assessment. Clin Oral Implants Res 2003;14:767-774.

113. Wallace SS, Froum SJ. Effect of maxillary sinus augmentation on the survival of endosseous dental implants. A systematic review. Ann Periodontol. 2003;8:328-343.

114. Pjetursson BE, Tan WC, Zwahlen M, Lang NP. A systematic review of the success of sinus floor elevation and survival of implants inserted in combination with sinus floor elevation. J Clin Periodontol. 2008;35(Suppl 8):216-240.

115. Del Fabbro M, Testori T, Francetti L, Weinstein R. Systematic review of survival for implants placed in the grafted maxillary sinus. Int J Periodontics Restorative Dent. 2004;24:565-577.

116. Del Fabbro M, Wallace SS, Testori T. Long-term implant survival in the grafted maxillary sinus: a systematic review. Int J Periodontics Restorative Dent. 2013;33:773-783.

How to cite this article: Testori T, Weinstein T, Taschieri S, Wallace SS. Risk factors in lateral window sinus elevation surgery. Periodontol 2000. 2019;81:91-123. https://doi. org/10.1111/prd.12286 